\title{
The presynaptic protein Mover is heterogeneously expressed across brain areas and synapse types
}

\author{
Dissertation \\ for the award of the degree "Doctor rerum naturalium" \\ of the Georg-August-Universität Göttingen \\ within the doctoral program IMPRS Neuroscience \\ of the Georg-August University School of Science (GAUSS)
}

Submitted by Rebecca Wallrafen

from Viersen, Germany 


\section{Thesis Committee}

Prof. Dr. Thomas Dresbach

Department of Anatomy and Embryology, University Medical Center Göttingen, Göttingen, Germany

Prof. Dr. Nils Brose

Department of Molecular Neurobiology, Max Planck Institute for Experimental Medicine, Göttingen, Germany

Dr. Wiebke Möbius

Electron Microscopy Core Unit, Max Planck Institute for Experimental Medicine, Göttingen, Germany

\section{Members of the examination board}

Referee: Prof. Dr. Carolin Wichmann

Institute for Auditory Neuroscience and InnerEarLab, University Medical Center Göttingen, Göttingen, Germany

$2^{\text {nd }}$ Referee: Prof. Dr. Ralf Heinrich

Department of Cellular Neurobiology, Schwann-Schleiden Research Center, Göttingen, Germany

$3^{\text {rd }}$ Referee: Prof. Jochen Staiger

Department of Neuoranatomy, University Medical Center Göttingen, Göttingen, Germany

Date of oral examination: 18th of February 2019 
"Life isn't about waiting for the storm to pass... It's about learning to dance in the rain!"

- Vivian Greene 
(This page intentionally left blank) 


\section{TABLE OF CONTENTS}

SUMMARY.

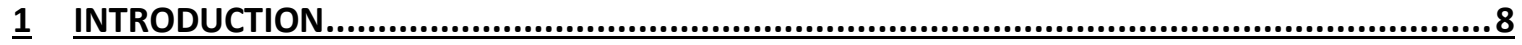

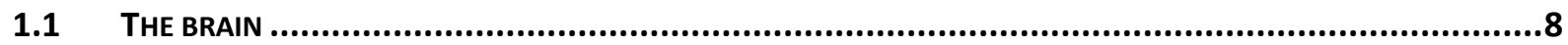

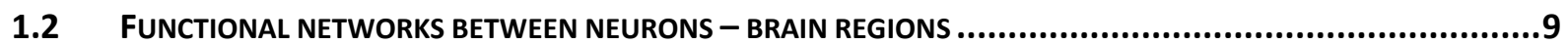

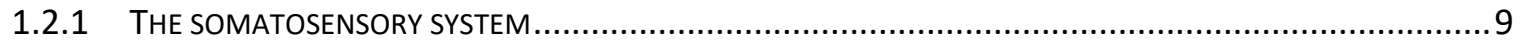

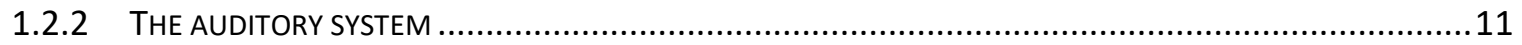

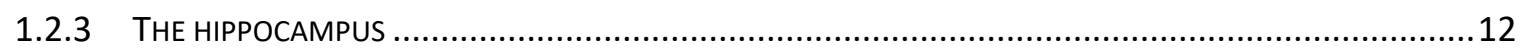

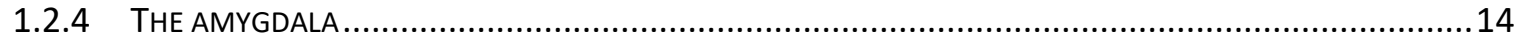

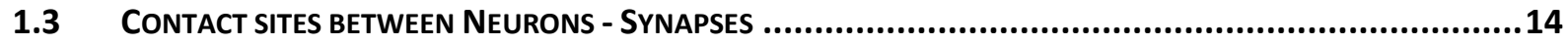

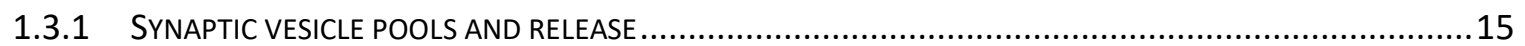

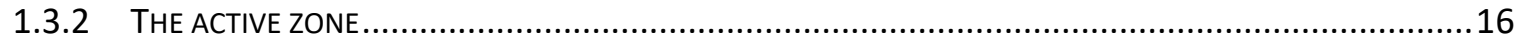

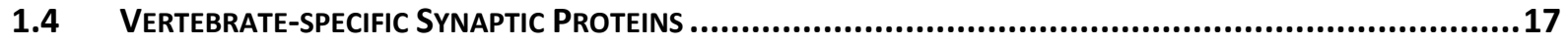

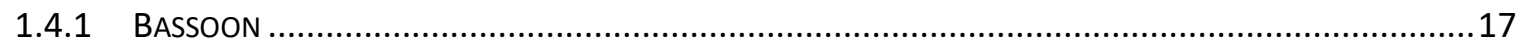

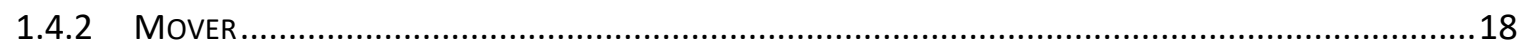

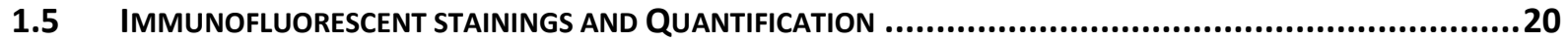

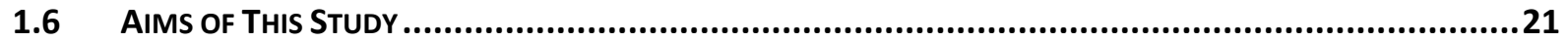

\section{$\underline{2}$ ESTABLISHING A QUANTITATIVE DISTRIBUTION ANALYSIS USING IMMUNOFLUORESCENT}

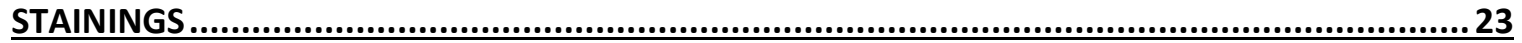

$\underline{3}$ THE PRESYNAPTIC PROTEIN MOVER IS HETEROGENEOUSLY DISTRIBUTED ACROSS BRAIN

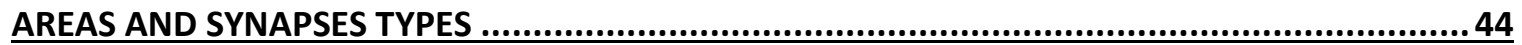

4 MOVER HAS DIFFERENTIAL EFFECTS ON SYNAPTIC-VESICLE-TO-ACTIVE-ZONE-DISTANCE AT ENDBULBS OF HELD AND INHIBITORY SYNAPSES TARGETING BUSHY CELLS IN THE VCN .........73

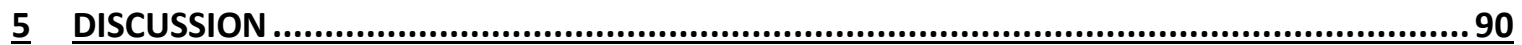

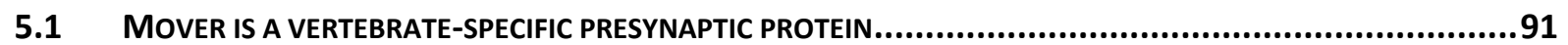

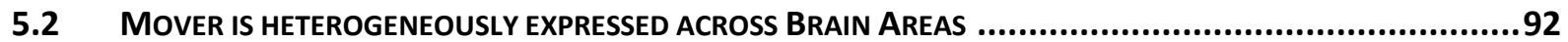


5.3 MOVER CAN BE DIFFERENTIALLY EXPRESSED WITHIN BRAIN AREAS ...............................................92

5.3.1 MOVER IS HETEROGENEOUSLY EXPRESSED WITHIN THE HIPPOCAMPUS ...............................................93

5.3.2 MOVER SHOWS A LAYER-SPECIFIC DISTRIBUTION IN THE SOMATOSENSORY SYSTEM............................ .94

5.3.3 MOVER LEVELS ARE HOMOGENEOUSLY HIGH ACROSS THE DIFFERENT AMYGDALOID NUCLEI ........................96

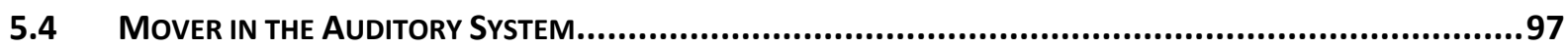

5.4.1 LOCALIZATION OF MOVER AND BASSOON AT THE AZ …....................................................... 98

5.4.2 MOVER MIGHT FUNCTION AS AN ADAPTER BETWEEN BASSOON AND SVS IN EXCITATORY SYNAPSESFEHLER! TEXTMARKE NICHT DEF

5.4.3 MOVER MIGHT MEDIATE SV DOCKING AT INHIBITORY SYNAPSES ........... FeHLER! TeXtMARKE NICHT DEFINIERT.

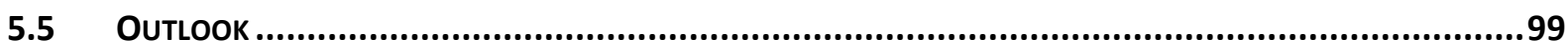

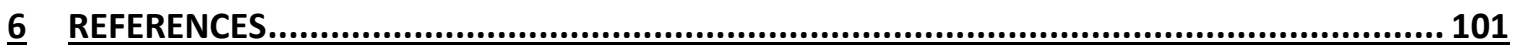

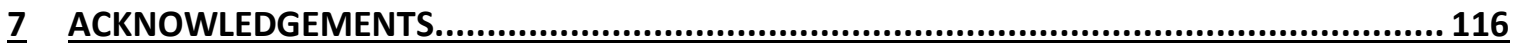

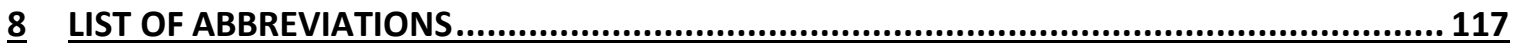

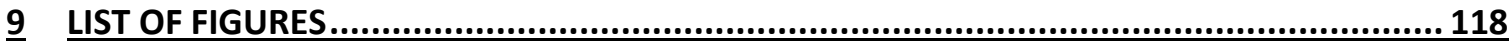

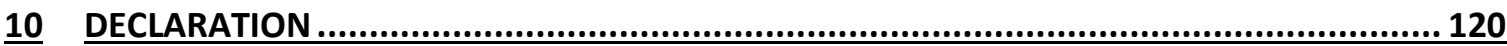

11 CURRICULUM VITAE .......................................FEHLER! TEXTMARKE NICHT DEFINIERT. 


\section{SUMMARY}

Synaptic nerve terminals are highly specialized sites where complex processes are taking place. The regulation of these complex processes is mediated by an intricate machinery of presynaptic proteins. While most of these proteins are evolutionarily conserved, a remarkably small number occurs only in vertebrates. They may increase the complexity and convey specialization to vertebrate synapses, thereby potentially bridging the gap between simple and complex behaviors. Among the vertebrate-specific proteins are the scaffolding molecules Bassoon and Piccolo, the synaptic vesicle associated protein Synuclein and Mover.

Mover is a small, synaptic vesicle attached phosphoprotein that was first discovered as a binding partner of the scaffolding molecule Bassoon. Its expression is regulated by activity. While most of its function remains unknown, knockout experiments showed that Mover buffers synaptic plasticity at the mossy fiber synapse in the hippocampus. Knockdown of Mover at the calyx of Held increased synaptic release probability and accelerated synaptic vesicle reloading.

Already in the first publication Mover was described to be heterogeneously expressed, and was found at subsets of synapses, while it seemed absent from others. In this study I have first established a quantitative immunofluorescence approach, comparing Mover fluorescence intensity to that of an internal reference marker, i.e. Synaptophysin, to determine the relative Mover abundance. I find that Mover is heterogeneously expressed, with high levels in some brain regions, such as the ventral pallidum, septal nuclei and the amygdala, and low levels relative to Synaptophysin in other brain regions, such as the primary motor cortex and the granular layer of the cerebellum. I also applied the quantification approach to the different layers of the hippocampus and find that Mover is enriched in layers that are associated with intra-hippocampal computation, and that Mover levels are low in input- and output layers.

Next, I characterized Mover at the endbulb of Held, i.e. first relay station of the binaural pathway which is responsible for hearing. I find that while absolute Mover levels are higher at inhibitory synapses contacting bushy cells than at endbulbs, the amount of Mover per synaptic vesicle is higher at endbulbs.

Together, the data confirm the heterogeneous distribution of Mover on three distinct levels: (1) across brain regions; (2) within single brain regions; (3) across synapse types. Its differential association with synapses on the level of brain areas, subregions and types of synapses renders Mover a candidate for a protein that generates synaptic heterogeneity. 


\section{INTRODUCTION}

\subsection{THE BRAIN}

The main function of the brain is the integration of sensory input with ongoing brain activity like memory and attention - to compute appropriate output. Its building blocks are neurons and non-neuronal cells, like glia, neuronal stem cells and cells associated with the circulatory system. The nervous system of an organism can be rather simple, consisting of only some 509 neurons in Caenorhabditis elegans, for example, or highly complex, as in vertebrates. The basic characteristics of the neurons are the same though, throughout the different levels of complexity of the entire nervous system: neurons are highly specialized, electrically excitable cells that transmit information from the periphery to the brain, form neuronal networks for the integration and computation of the information, and control output by projecting back into the periphery. They are unique to the nervous system. In general, each neuron consists of three distinct cell compartments: dendrites, soma and axon. The dendrites are processes originating from the soma and form the main input site. They receive input from many other neurons, usually both from local circuitry as well as long-range projections. While each neuron typically is equipped with only one axon, it can have several dendrites, which form elaborate tree-like structures. Signals are integrated at the soma, and in case a certain threshold is passed, the signal gets transmitted along the axon, a thin process which can span several microns up to a meter (Brodal, 2010). Neurons are interconnected at specialized sites called synapses. In complex nervous systems, one neuron is connected to thousands of other neurons. Synapses are typically formed between the axonal bouton of one neuron as the presynaptic terminal and the dendrite of a second neuron as the postsynaptic site. Other types of synapses, such as axoaxonic or axo-somatic connections, are described, but less frequent (Brodal, 2010).

As the input into the brain usually is multi-modal, i.e. coming from different sensory systems at the same time, a certain degree of segmentation and structure is required for the brain to correctly compute adequate responses to the different stimuli. This segmentation is achieved through the formation of neuronal networks that transmit signals from only one sensory modality to the target region in the brain. These networks can be local, i.e. only interact within the brain region, but they can also span different parts of the brain and range between different brain areas. The information can then get forwarded to higher brain areas, where signals from 
the different input sources are integrated. Based on this functional aspect, the brain can be divided into different, specialized areas.

While mice differ from humans in many aspects at first glance, genetically speaking we are 97.5\% similar (Mural et al., 2002). This genetic similarity yields the advantage, that mice can be used as a model organism for research. Additionally, the morphology of the mouse brain concerning brain areas and (local) networks is comparable to that of the human brain. This genetic and morphological similarity makes mice a suitable model organism for brain research. Henceforth, all information presented in this thesis will be about the mouse nervous system, unless stated otherwise.

\subsection{FUNCTIONAL NETWORKS BETWEEN NEURONS - BRAIN REGIONS}

In general, the brain is divided into grey and white matter. In the cortex, which consists of grey matter, the cell bodies and dendrites of neurons can be found, while the white matter is mostly made up of axons and non-neuronal cells, such as oligodendrocytes. The cell bodies in the cortex are arranged in layers. Different types of cortices can be histologically distinguished: while the neocortex contains 6 cell body layers, the allocortex only has three or four distinct layers. Typical neocortical structures are the sensory cortices and motor cortex, while the olfactory system and hippocampus are the most prominent allocortical structures. The axons in the white matter are wrapped in sheaths of fat and proteins formed by oligodendrocyte processes, called myelin, to increase the conductance speed of signal transmission. The white matter appears white due to the myelin, while the cell bodies give the grey matter a darker appearance. Together, cortical and subcortical structures form functional networks, some of which are relevant for this study and thus will be described in more detail.

\subsubsection{The somatosensory system}

To sense their environment, mice rely on their sense of touch, and especially on their whiskers. When the whisker touches an object in the mouse's surrounding, mechanoreceptors in the whisker pad get activated. Information is then relayed to the soma of the cell, which is located in the trigeminal ganglion. From there, cells transmit the information to the trigeminal nucleus in the brain stem, which projects to the thalamus. From the thalamus, information gets transferred to the somatosensory cortex (S1), which, due to its unique organization, is also called barrel cortex (Figure 1A). S1 is a typical example of a neocortical structure, and therefore consists of 6 

are

\section{A From Whisker to Cortex}

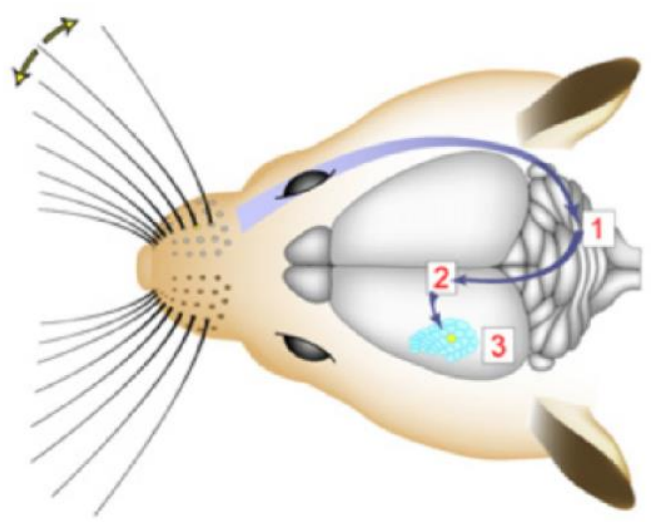

B Thalamocortical connectivity

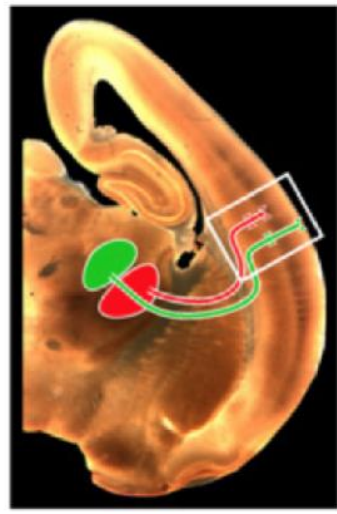

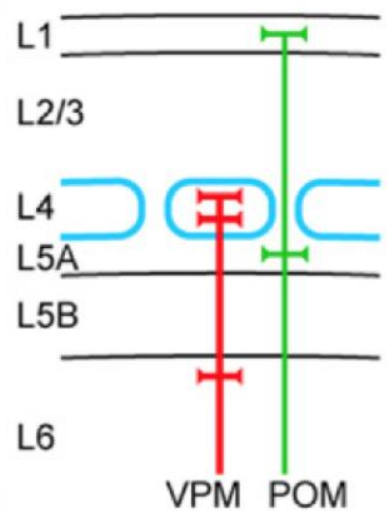

Figure 1: The somatosensory system in mice. (A) Representation of the information flow, which is transferred from the whisker to the brain stem (1) to thalamic nuclei (2) to the primary somatosensory cortex $(S 1 ; 3)$. (B) Description of the two distinct pathways: information transmitted via the lemniscal pathway (red) reaches the ventral posteromedial nucleus (VPm) of the thalamus, which targets the barrels (blue) in layer IV and, to some extent, cells located at the border of layer $\mathrm{Vb} / \mathrm{VI}$. The paralemniscal pathway (green) transmits information via the posteromedial nucleus (POm) of the thalamus and projects to layer Va and I. Reprinted from Neuron, 56, C. Petersen, 'The Functional Organization of the Barrel Cortex', page 340, 2007, with permission from Elsevier.

represented somatotopically along the whole pathway, meaning that one barrel in a row of S1 corresponds to the whisker in the same row of the whisker pad on the animal's snout (Welker \& Woolsey, 1974). Consequently, the barrels are arranged in columns and rows, just like the whiskers (Schubert, Kötter \& Staiger, 2007). Different types of information obtained from the whiskers are transferred along the lemniscal and the paralemniscal pathway, two separate routes of information transmission (Figure 1B). While the touch-mediating function of the lemniscal pathway has been described in detail (e.g. Nicolelis, 2005; Yu et al., 2006), the exact function of the paralemniscal pathway remains unknown. Functions include but seem not to be limited to modulation of the lemniscal pathway (Ahissar, Sosnik \& Haldarilu, 2000) and pain sensation (Frangeul et al., 2014). Anatomically, both pathways have been thoroughly characterized.

Information along the lemniscal pathway reaches the ventral posteromedial nucleus (VPm) of the thalamus from the trigeminal nucleus (Figure 1B, red). Fibers from the VPm mainly project to the barrels in layer IV. Recently, it was discovered that these fibers additionally project to the border between layers V and VI, albeit to a minor extent (Egger et al., in press; talk at SFN 2018). From layer IV the signal gets transmitted to layer II/III, where it gets integrated with signals 
coming from neighboring columns and rows. Layer II/III pyramidal neurons project, among others, to layer $\mathrm{Vb}$ of the same column, and from there the signal gets transferred to other brain regions.

Paralemniscal input reaches the thalamus at the posteromedial nucleus (POm; Figure 1B, green). POm neurons target mainly the secondary somatosensory cortex, that has a modulating function. They also target layer Va in the primary somatosensory cortex, and to a smaller extent layer I (Bosman et al., 2011). By targeting layer I of S1, paralemniscal input may mediate cortical firing, as layer $I$ is known to regulate cortical excitability and sensory response magnitude and duration (Castejon et al.,, 2016; Shlosberg, Amitai, \& Azouz, 2006).

\subsubsection{The auditory system}

Another sensory modality that mice greatly rely on is hearing. Sound reaches the ear, where it is sensed by hair cells in the cochlea. From the cochlea, the axons of spiral ganglion neurons form the auditory nerve (AN), which projects to the cochlear nucleus (Figure 2). There, the auditory nerve fibers (ANFs) target bushy cells (BCs), one type of principal cells of the ventral cochlear nucleus (VCN) and form synapses called endbulbs of Held. This is the first relay station of the socalled binaural pathway, that mediates fast and precise processing of auditory information (Young \& Oertel, 2003, 2010). The binaural pathway by far is not the only pathway there is in the auditory system. It is, however, the most prominent and most researched one. There are many other "short cuts" and relays, which will not be in the focus of this study. In the binaural pathway, the BCs target the ipsilateral lateral olivary complex and the contralateral medial nucleus of the trapezoid body (Alibardi, 1998; Suneja et al., 1995). There they form giant synapses known as the calyx of Held (Borst \& van Hoeve, 2012). From there, the signal gets transmitted to the inferior colliculus, which projects to the medial geniculate body. After this last subcortical relay station, the information reaches the auditory cortex. Focus in this study will be given to the first relay station however, where we find the BCs receiving endbulbs of Held and inhibitory input, among others from the dorsal cochlear nucleus.

BCs can either be spherical or globular. They are organized in a tonotopic manner within the VCN, with cells coding low frequencies located in the ventral rostral part of the VCN, and cells coding high frequencies located more caudally and dorsally (Young \& Oertel, 2003). The tonotopy is visualized by the rainbow color code in Figure 2: Areas of one color are wired together and represent signal transmission from similar frequency ranges. BCs receive input from unmyelinated type I ANFs, which form endbulb of Held synapses. One characteristic 
feature of endbulb synaptic physiology is that it is highly plastic because of its strong depression (Yang \& Xu-Friedman, 2009).

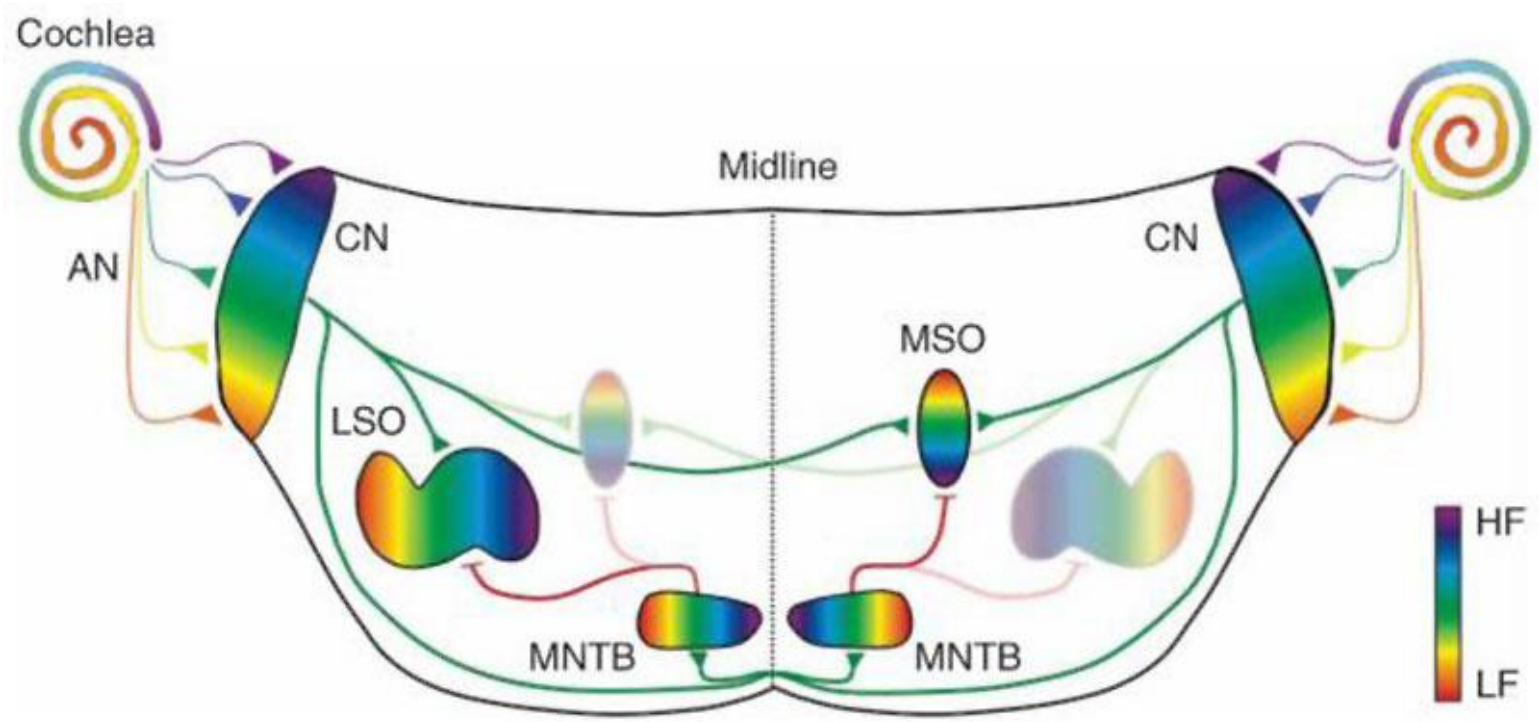

Figure 2: Schematic overview on the auditory system of mice. Sound reaches the cochlea and is transferred to the cochlear nucleus (CN), where auditory nerve (AN) fibers synapse onto bushy cells, forming the endbulbs of Held. Bushy cells project to the ipsilateral lateral superior olive and the contralateral medial nucleus of the trapezoid body (MNTB), where they form the calyx of Held. Reprinted from Nature Neuroscience, 12, K.Kandler, A. Clause, J. Noh, 'Tonotopic reorganization of developing auditory brainstem circuits', page 712, 2009, with permission from Springer Nature.

\subsubsection{The hippocampus}

The hippocampus was first described by Julius Ceasar Aranzi in 1587, a Venetian anatomist. He compared the shape of the allocortical structure initially to that of a silkworm, later to a sea

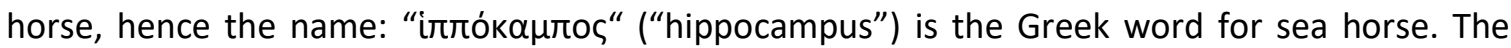
hippocampus consists of the hippocampus proper, and the dentate gyrus (DG). The hippocampus proper is also called Ammon's horn, or Cornu Ammonis (CA), where the subfields derive their name from: CA1, CA2, CA3 and CA4. The connectivity of the hippocampus has been studied extensively and described in much detail (Figure 3). While CA1 and CA3 are often focused on, there is a growing body of literature also describing CA2, which is a small subfield located between CA3 and CA1. CA4 is often described as a deeper layer of the DG.

The hippocampus receives its input from the entorhinal cortex. From there, fibers project mainly to the granule cells of the DG, while a minority of the projections directly targets CA3 and CA1 pyramidal neurons. The connection to the DG granule cells is called the perforant path. Granule cells in the DG send their axons to the apical dendrites of CA3 pyramidal cells and form the mossy fibers synapses, giant synaptic terminals that can harbor up to 16000 SVs (Andersen et al., 2007; Rollenhagen et al., 2007; Rollenhagen \& Lübke, 2010). These synapses are also known for 
their low initial release probability and strong synaptic facilitation (Hallermann et al., 2003; Rollenhagen et al., 2007). This means that they react rather weakly to an initial stimulus, but that

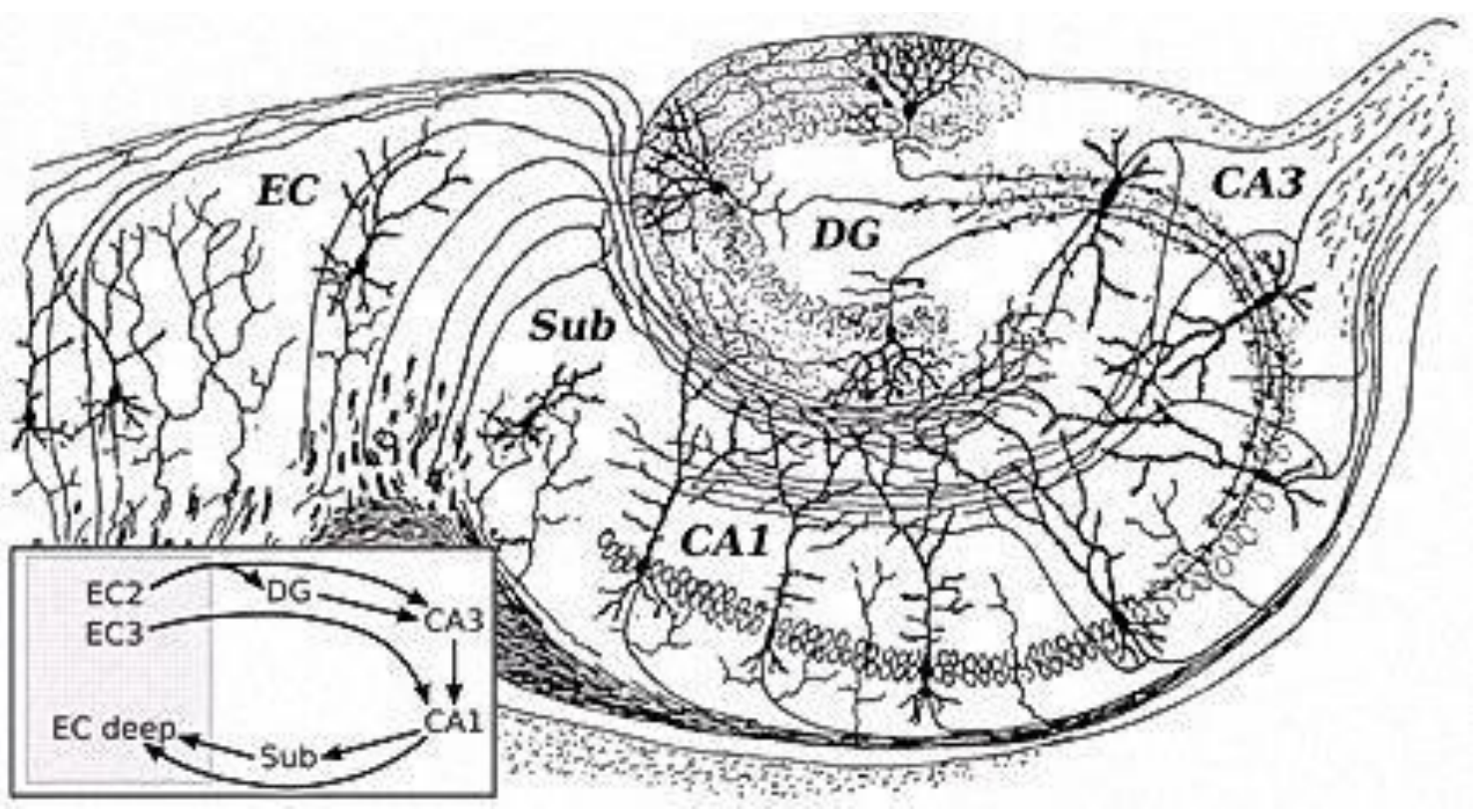

Figure 3: Drawing of the hippocampus by Santiago Ramón y Cajal (1911, Public Domain). The basic circuitry of the hippocampus is shown in a schematic representation in the insert in the left lower corner.

the response increases with a second stimulus, if this stimulus occurs within a certain range of time. CA3 pyramidal neurons send their axons to the dendrites of CA1 pyramidal neurons. These fibers are called the Schaffer collaterals. CA1 neurons then in turn transmit the signal back to the entorhinal cortex, either directly or via a relay station called the subiculum. As there are three relay stations within the hippocampus via which a signal gets transmitted (DG $\rightarrow$ CA3 $\rightarrow$ CA1), the whole system is also called the trisynaptic pathway (Andersen et al., 2007).

Like most cortices, the hippocampus shows a layered structure. The first layer of the hippocampus is called the stratum oriens. It contains interneurons and the basal dendrites of the pyramidal neurons, and thus some CA3 to CA3 and CA3 to CA1 connections can be found there. The next layer is the pyramidal cell layer, the stratum pyramidale. This layer harbors the cell bodies of pyramidal neurons. Mainly inhibitory connections originating from interneurons can be found in this layer. CA3 and CA1 differ in the next layer: While in CA1 the stratum radiatum is found next to the pyramidal cell layer, in CA3 there is an intermediate layer, the stratum lucidum. This is where the mossy fibers from DG can be found. As these fibers are unmyelinated, this layer has a clear appearance, hence the name. In CA3, the stratum radiatum can be found adjacent to the stratum lucidum. This layer contains interneurons, associational fibers (CA3 to CA3 connections) and the Schaffer collaterals (CA3 to CA1 connections). Additionally, the 
stratum radiatum contains many recurrent connections. The most superficial layer is the stratum lacunosum-moleculare, which contains mainly interneurons and inhibitory connections (Andersen et al., 2007).

Functionally, the hippocampus has been related to memory formation and consolidation from short-term to long-term memory, mainly of episodic memory, i.e. autobiographical events. Additionally, the hippocampus is involved in spatial recognition and spatial coding, as it harbors place cells. The hippocampus is therefore also believed to harbor the brain's "cognitive map" (O’Keefe \& Nadel, 1978).

\subsubsection{The amygdala}

While input to the hippocampus is clearly defined and restricted to originate from one brain area (the entorhinal cortex) there are other brain regions that have much more divers input. One of these regions is the amygdala (Figure 4). Strictly speaking, "the amygdala" does not exist: instead of one homogeneous brain region, the amygdala is an assortment of different nuclei (for simplicity, however, this group of nuclei is usually referred to as the amygdala). The different amygdaloid nuclei have been linked to a variety of processes and functions. The basolateral amygdala (BL) is associated with fear-conditioning and anxiety-related behavior (LeDoux et al., 1990). Other nuclei have been described to modulate other processes, such as hormone secretion (Eleftheriou \& Zolovick, 1967). The medial nucleus especially has been described to mediate and regulate emotional and sexual behavior (Fernandez-Fewell \& Meredith, 1994; Kondo, 1992). It receives its main input from the accessory olfactory bulbs, which is responsible for the detection of pheromones, among others (Trinh \& Storm, 2003). Both excitatory, i.e., glutamatergic, and inhibitory, i.e., GABAergic, neurons found in the medial nucleus project to the hypothalamus, but there is a subpopulation of GABAergic interneurons which provide feedforward inhibition locally within the medial nucleus.

Notably, in humans, the amygdala has also been implicated to play a role in psychiatric disorders, such as trauma in general and posttraumatic stress disorder (PTSD) in particular (Mahan \& Ressler, 2012).

\subsection{CONTACT SITES BETWEEN NEURONS - SYNAPSES}

To form neuronal networks as described above, neurons need to be able to communicate with each other. This communication is based on the transmission of signals between one neuron and 
its partner and can either be electric or through chemical molecules called neurotransmitters. While electric synapses enable bidirectional communication (i.e., there is no "sender" and no

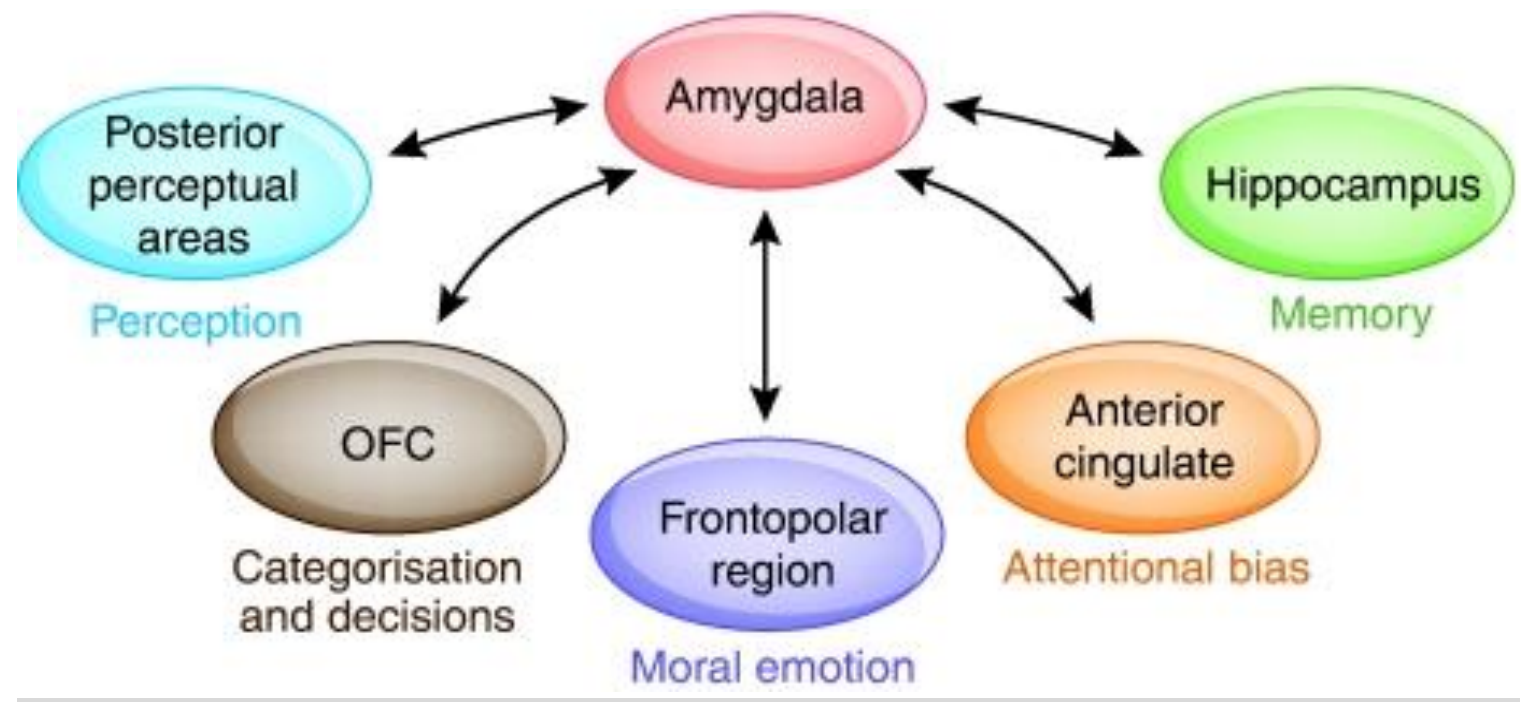

Figure 4: Schematic overview of the input to the amygdala. Input comes from all over the brain. Reprinted from Neuropsychopharmacology, 36, R. Elliott, R. Zahn, J. F. W. Deakin, I. M. Anderson, 'Affective Cognition and its Disruption in Mood Disorders', page 160, 2011, with permission from Springer Nature.

"addressee" per se, both sides can fulfil both functions), chemical synapses usually function only in one direction. They can be highly adaptable, which is called plasticity. In this study, chemical synapses will be in the spotlight. Upon the arrival of a stimulus - typically an action potential transmitter molecules will be released from the presynaptic terminal, diffuse into the synaptic cleft, and reach the membrane of the receiving neuron, where they can interact with receptor molecules, which in turn start a downstream reaction in the postsynaptic neuron.

The neurotransmitter molecules are packed in vesicles, which are about $40 \mathrm{~nm}$ in diameter ( $\mathrm{Qu}$ et al., 2009). They are arranged into different pools in the presynaptic terminal (Alabi \& Tsien, 2012), which I will now describe in more detail.

\subsubsection{Synaptic vesicle pools and release}

Typically, three different pools of SVs can be found in the presynaptic compartment: the resting pool (RP), the recycling pool and the readily releasable pool (RRP; Rizzoli \& Betz, 2005). Which SV belongs to which pool depends on the definition of the pool: either based on the spatial location of the SV in the presynaptic terminal, or based on functional aspects, such as release probability of the SV (Alabi \& Tsien, 2012). SVs that belong to the RP are located furthest from the presynaptic membrane, and have the lowest release probability, as mobilization of this pool 
takes time. SVs in the recycling pool and RRP have higher release probabilities. The RRP consists of SVs that are already docked to the presynaptic membrane (Imig et al., 2014). This docking is mediated by the SNARE proteins (Soluble $\mathrm{N}$-ethylmaleimide sensitive factor attachment protein receptor): Vesicle-associated membrane protein (VAMP, also known as Synaptobrevin, Trimble, Cowan, \& Scheller, 1988), Syntaxin (Bennett et al., 1992) and Synaptosomal-associated protein of 25kDA (SNAP25, Oyler et al., 1989). VAMP is associated with the SV membrane and forms a lose core complex with Syntaxin and SNAP25, which are attached to the presynaptic membrane. The core SNARE complex, together with other proteins, including Munc13 and Munc18, brings the SV membrane and the presynaptic cell membrane into close proximity. Munc18 binds to Syntaxin, thereby starting the process of SV fusion (Ma et al., 2012). Munc13 on the other hand is involved in rendering SVs release-ready (before fusion can happen), which is called "priming" (Varoqueaux et al., 2002). Both Munc-isoforms are required for neurotransmission, as deletion of either results in a total loss of SV fusion and transmitter release (Varoqueaux et al., 2002; Verhage et al., 2000). The docked SVs are the ones that are released (and depleted) first upon the arrival of a stimulus (Rosenmund \& Stevens, 1996; Schneggenburger et al., 2002; Von Gersdorff et al., 1996), thereby contributing most to the strength of the synapse (Dobrunz \& Stevens, 1997; Waters \& Smith, 2002). The recycling pool replenishes the RRP after stimulus onset, which requires additional transitional processes (i.e., docking and priming). The replenishment rate generally is the limiting factor during persistent synaptic activity and greatly influences neuronal plasticity (Alabi \& Tsien, 2012).

When an action potential reaches the synaptic bouton, the depolarization of the terminal leads to the opening of voltage-gated $\mathrm{Ca}^{2+}$-channels (VGCCs, Dolphin, 2009) and influx of $\mathrm{Ca}^{2+}$ into the presynaptic terminal. The elevated $\mathrm{Ca}^{2+}$-concentration causes a tightening of the SNAREcomplex, which exerts tension on the two membranes, and creates a fusion pore. Through this pore, the neurotransmitter molecules can diffuse into the synaptic cleft and interact with the neurotransmitter receptors located in the postsynaptic membrane. $\mathrm{Ca}^{2+}$-channels are not localized randomly in the presynaptic membrane, but are tethered to the membrane by Rab3interacting molecules (RIM; Kaeser et al., 2011). These specialized sites in the presynaptic membrane, where SV fusion is observed, are called active zones (AZ).

\subsubsection{The active zone}

At the $A Z$, a plethora of molecules tightly regulates the SV cycle, from docking, priming, fusion to re-uptake and refilling of the SVs. This electron-dense mesh of proteins is called the cytomatrix of the active zone (CAZ). Among the proteins forming the CAZ are Munc13, Piccolo (also called 
Aczonin), RIM and RIM-binding proteins (RIM-BPs), ELKS/CAST and Bassoon. They are arranged in a precise manner, allowing for the localization of an AZ exactly opposite the postsynaptic density.

While all CAZ-proteins have unique functions, their interplay is important for the AZ to fulfil its function: mediating the fusion of SVs and releasing neurotransmitter into the synaptic cleft. The organization of the different proteins enables SVs to be brought into close proximity to $\mathrm{Ca}^{2+}$ channels (Ackermann et al., 2015), allowing SV fusion to happen shortly after opening of the channels. The regions where SVs and $\mathrm{Ca}^{2+}$-channels are clustered are also called microdomains (Chad \& Eckert, 1984; Neher, 1998; Simon \& Llinás, 1985). In some synapses, they are clustered in such close proximity that they are even called nanodomains (Bucurenciu et al., 2008). This concept of clustering is one explanation for the different release probabilities of SVs: The closer an SV is located to a $\mathrm{Ca}^{2+}$-channel, the less calcium influx is needed to induce fusion of the SV. This means that SVs that are closest to a $\mathrm{Ca}^{2+}$-channel have the highest chance of being released, and thus the highest release probability. Other factors play a role as well, such as the intrinsic $\mathrm{Ca}^{2+}$-sensitivity of the sensor, which will be discussed in the context of superpriming later on.

\subsection{VERTEBRATE-SPECIFIC SYNAPTIC PROTEINS}

The processes happening at the $A Z$ are tightly regulated by a highly complex core machinery consisting of many proteins. Most of these proteins are evolutionarily conserved and can be found even in the simplest organism. Exocytosis, for example, is mediated by the SNARE proteins already in Saccharomyces cerevisiae, more commonly known as "baker's yeast". A remarkably small number, however, occurred together with the evolution of vertebrates.

\subsubsection{Bassoon}

Among the vertebrate-specific proteins is the scaffolding molecule Bassoon. It is a large protein, consisting of 3938 amino acids, with a molecular weight of $420 \mathrm{kDa}$, that is part of the CAZ (Gundelfinger \& Fejtova, 2012; tom Dieck et al., 1998). Bassoon is trafficked together with another vertebrate-specific protein called Piccolo on Golgi-derived vesicles (Dresbach et al., 2006). At the Golgi apparatus, it shows a stretched orientation, with the $\mathrm{N}$-terminus located in the Golgi membrane and the C-terminus sticking out into the cytosol. This conformation is lost during trafficking (Ghelani, 2016), but re-established at the synapse, where it changes orientation: the $\mathrm{C}$-terminus is now close to the presynaptic membrane, while the $\mathrm{N}$-terminus 
sticks into the synaptic bouton and is therefore localized between SVs (Dani et al., 2010; Limbach et al., 2011; Sanmartí-Vila et al., 2000; Tsuriel et al., 2006). How exactly this orientation change comes about remains unknown.

At the $A Z$, Bassoon binds to many other proteins involved in the regulation of presynaptic activity, such as CtBP1 and CtBP2/RIBEYE (tom Dieck et al., 2005), Munc13 (Wang et al., 2009), CAST/ELKS (Takao-Rikitsu et al., 2004), RIM (Wang et al., 2009). It also binds to Piccolo (Dresbach et al., 2006). Together, the two proteins are involved in presynaptic assembly, maintenance and integrity by regulating SV clustering, without directly participating in exocytosis (Mukherjee et al., 2010). Additionally, Bassoon stabilizes synapses by reducing proteasomal degradation (Waites et al., 2013) and regulating autophagy (Okerlund et al., 2017).

Disruption of Bassoon in the endbulb of Held synapse in the auditory system was shown to cause a slowdown in SV replenishment (Mendoza Schulz et al., 2014). Interestingly, the RRP size was increased, as well as the release probability of SVs. This increase in release probability can potentially be explained by the observed downregulation of Mover, the protein of interest in this study.

\subsubsection{Mover}

Mover is another vertebrate-specific protein that was identified as a binding partner of Bassoon in a yeast-2-hybrid assay (Kremer et al., 2007). The binding site is located in the C-terminal region of Bassoon, but the exact position remains unknown. Additionally, Mover was identified in two other studies. In a 2D gel electrophoresis study analyzing synaptic proteins, Mover was initially called Synaptic vesicle associated protein of 30kDa (SVAP30, Burré et al., 2006). In a second study, it was found as a homolog of the protein Transformation related protein 63 (and thus called Transformation related protein 63 regulated like, Tprgl, Antonini et al., 2008). In addition to its expression in the nervous system, Mover can be found in various other tissues, such as liver, skin and testis (Antonini et al., 2008; Kremer et al., 2007). In neurons, Mover has been found to be attached to synaptic SVs (Ahmed et al., 2013).

In contrast to Bassoon, Mover is a relatively small protein. It consists of only 266 amino acids and weighs around 30kDa. In order to be trafficked correctly, Mover self-interacts and forms oligomers (Ahmed et al., 2013). It was hypothesized that the Sac2 domain of Mover is necessary for oligomerization (Hsu et al., 2015), but research from our lab showed that this domain alone is not sufficient (Akula, 2015). Additionally, Mover has several phosphorylation sites, and phosphorylation is required for the interaction with SVs (Ahmed et al., 2013). Another important 
characteristic of Mover is its activity dependence: after tetrodotoxin treatment of hippocampal cell culture, Mover levels were decreased (Kremer, 2008), while they were increased after treatment with forskolin, an activator of the adenylyl cyclase (Moritz Arndt, personal communication). Mover was downregulated in endbulbs of Held after Bassoon disruption (Mendoza Schulz et al., 2014), which renders mice deaf, another implication for an activitydependent expression.

Electrophysiological analysis of the mossy fiber terminals in a knock out model of Mover has shown that while basic features of synaptic transmission remain unchanged, loss of Mover leads to an increase in short-term plasticity, i.e. increased frequency facilitation (Viotti, 2017; Viotti et al., unpublished). Knock down of Mover at the calyx of Held, a highly specialized synapse in the auditory pathway, increased synaptic release probability by increasing the $\mathrm{Ca}^{2+}$-sensitivity of release (Körber et al., 2015). This change is best explained by an altered calcium sensitivity of the intrinsic $\mathrm{Ca}^{2+}$-sensor, and has therefore been connected to superpriming (Körber \& Kuner, 2016). Superpriming is a quite recent concept. Superprimed SVs are part of the RRP, but show an increased release probability (Lee et al., 2013; Schlüter et al., 2006; Taschenberger et al., 2016). How this increase comes about remains controversial: Ishiyama et al. (2014) argue that it is due to shorter vesicle-channel distances, while the majority of researchers believe it to be a maturation effect of the SV's intrinsic $\mathrm{Ca}^{2+}$-sensitivity (Basu et al., 2007; Lee et al., 2013; Michelassi et al., 2017; Taschenberger et al., 2016).

Interestingly, using immunogold electron microscopy, Mover was detected on only $16 \%$ of SVs, while Synaptophysin, another SV-attached protein (Navone et al., 1986), was present on all SVs (Ahmed et al., 2013). While it is tempting to deduce a heterogeneous distribution from these results, the numbers have to be handled with care: the results were obtained by analyzing the synaptic vesicle fraction obtained from a whole rat brain homogenate. That means that all SVs were analyzed together, without distinguishing different brain regions or synapse types. It is therefore impossible to distinguish between the following two scenarios: (1) The number of Mover molecules per synapse is constant but lower than that of Synaptophysin, i.e. Mover is present in every synapse, but only on a subset of SVs; (2) The number of Mover molecules varies between synapses, i.e. some synapses have high Mover levels, while others have low to no Mover. As one of the first studies describing Mover in the nervous system already described it to be "differentially localized to subsets of synapses" (Kremer et al., 2007), we hypothesized that option 2, i.e. a heterogeneous distribution of Mover throughout the brain, is the underlying cause for the low percentage of Mover-positive SVs. To corroborate our hypothesis, we decided 
to quantify the heterogeneous distribution of Mover across the adult mouse brain using an immunofluorescence approach.

\subsection{IMMUNOFLUORESCENT STAININGS AND QUANTIFICATION}

To localize (synaptic) proteins in their physiological surroundings, we take advantage of immune reactions, which can be visualized using fluorophores. Primary antibodies are generated against the protein of interest by immunizing a host organism (e.g. mice, guinea pigs or rabbits) with an antigen, i.e. the protein of interest. The host organism then produces antibodies against the antigen, which can be isolated from the blood (in case of a polyclonal antiserum) or from immune cells (like spleen cells or lymphocytes in case of monoclonal antibodies). The primary antibodies can either be coupled directly to a fluorophore (direct immunofluorescence), or a secondary antibody (indirect immunofluorescence) can be used, which in turn is coupled to a fluorophore. The secondary antibody is raised in a different species than the primary antibody (e.g. goat or donkey) and detects all antibodies from one species (in contrast to the primary antibody, which is selective for the protein against which it was raised). In contrast to chromogenic staining methods, immunofluorescence allows for the detection of more than one protein at the time. For that, primary antibodies from different host species are used, which then are detected by secondary antibodies labeled with fluorophores of different wavelengths.

When employing Immunofluorescent techniques, one has to take into consideration certain caveats that are inherent to the technique. Even when treating the slices with the same solutions for the same incubation times, the labeling efficiency can differ, and slices can show a variability in staining intensity. To circumvent this inherent variability, we decided to quantify our protein of interest - Mover - relative to an internal reference marker and relative to the overall intensity across the hemisphere. This allows for the compensation of fluorescence intensity variability across different slices.

When quantifying the distribution of a protein that is localized to a specific cell compartment the synapse, in our case - we have to consider the distribution of the cell compartment itself across the brain. Regions with a high synaptic density will naturally yield a higher staining intensity for synaptic proteins than regions with only very few synapses, like cell body layers. This uneven distribution of the cell compartment can severely influence the interpretation of the results, yielding a false sense of heterogeneity. To compensate for this second caveat, we chose Synaptophysin as our internal reference marker. Synaptophysin is a presynaptic protein, which is present on all SVs and absent from other types of vesicles in the neuron, like dense core vesicles 
(Navone et al., 1986). While its definite synaptic function remains unknown, Synaptophysin has been described to regulate activity-dependent synapse formation in hippocampal neuronal cell culture (Tarsa \& Goda, 2002). Assuming that the labeling efficiency across one slice is constant, using Synaptophysin as the internal standard allows us to compensate for synaptic size and density: the more synapses are present in a certain region, the higher the Synaptophysin fluorescence intensity. The same holds true for the size of a synapse: the number of SVs scales with the size of the synapse, and thus also the number of Synaptophysin molecules. Taking the ratio between the Mover fluorescence intensity and the Synaptophysin fluorescence intensity therefore allows us to determine the amount of Mover relative to the number of SVs.

\subsection{AIMS OF THIS STUDY}

We have developed a quantification approach that allows for the quantitative analysis of a protein across brain regions and subregions and circumvents the inherent variability of immunofluorescent stainings by yielding a ratio rather than absolute values. The method has been accepted in the Journal of Visualized Experiments on the $21^{\text {st }}$ of September 2018 and will be published shortly (Wallrafen, Dresbach \& Viotti, in press). The manuscript can be found in Chapter 2 - ESTABLISHING A QUANTITATIVE DISTRIBUTION ANALYSIS USING IMMUNOFLUORESCENT STAININGS.

We employed this technique to quantify the distribution Mover across the adult mouse brain in 16 different brain areas. We show that there is a striking heterogeneity of the Mover distribution of three levels: between brain regions, within single areas and across synapse types. The manuscript describing this part of the project was published in Frontiers in Neuroanatomy on $13^{\text {th }}$ of July (Wallrafen \& Dresbach, 2018) and can be found in Chapter 3 - THE PRESYNAPTIC PROTEIN MOVER IS HETEROGENEOUSLY DISTRIBUTED ACROSS BRAIN AREAS AND SYNAPSES TYPES.

Additionally, we apply our quantification approach of Mover relative to Synaptophysin, i.e. the amount of Mover per SV, in the auditory pathway, where we focus on the first synaptic relay station, the VCN and its principal cells, the BCs. We find that while the absolute Mover fluorescence intensity is higher at inhibitory synapses contacting BCs compared to excitatory endbulbs of Held, the amount of Mover per SV is significantly higher in endbulbs than in inhibitory synapses (Wallrafen et al., in preparation). The manuscript presenting these results, among others, can be found in Chapter 4 - MOVER HAS DIFFERENTIAL EFFECTS ON SYNAPTIC-VESICLE-TOACTIVE-ZONE-DISTANCE AT ENDBULBS OF HELD AND INHIBITORY SYNAPSES TARGETING BUSHY CELLS IN THE VCN. 
Wallrafen, 2019 


\section{ESTABLISHING A QUANTITATIVE DISTRIBUTION ANALYSIS USING IMMUNOFLUORESCENT STAININGS}

Journal of Visualized Experiments, in press

\section{ABSTRACT}

"The presence, absence or levels of specific synaptic proteins can severely influence synaptic transmission. In addition to elucidating the function of a protein it is vital to also determine its distribution. Here we describe a protocol employing immunofluorescence, confocal microscopy and computer-based analysis to determine the distribution of the synaptic protein Mover (also called TPRGL or SVAP30). We compare the distribution of Mover to that of the synaptic vesicle protein Synaptophysin, thereby determining the distribution of Mover in a quantitative manner relative to the abundance of synaptic vesicles. Notably, this method could potentially be implemented to allow for comparison of the distribution of proteins using different antibodies or microscopes or across different studies. Our method circumvents the inherent variability of immunofluorescent stainings by yielding a ratio rather than absolute fluorescence levels. Additionally, the method we describe enables the researcher to analyze the distribution of a protein on different levels: from whole brain slices to brain regions to different subregions in one brain area, such as the different layers of the hippocampus or sensory cortices. Mover is a vertebrate-specific protein that is associated with synaptic vesicles. With this method we show that Mover is heterogeneously distributed across brain areas, with high levels in the ventral pallidum, the septal nuclei and the amygdala, and also within single brain areas, such as the different layers of the hippocampus." (Wallrafen, Dresbach \& Viotti, in press) 


\section{AUTHORS:}

Rebecca Wallrafen, Thomas Dresbach, Julio S. Viotti

Institute of Anatomy and Embryology, University Medical Center Göttingen, Göttingen, Germany

\section{CORRESPONDING AUTHOR:}

Rebecca Wallrafen (Rebecca.wallrafen@med.uni-goettingen.de)

\section{KEYWORDS:}

Immunofluorescence, confocal microscopy, quantification, synaptic proteins, mouse, Mover, distribution

\section{SHORT ABSTRACT:}

Here, we describe a quantitative approach to determining the distribution of a synaptic protein relative to a marker protein using immunofluorescence staining, confocal microscopy, and computer-based analysis.

\section{INTRODUCTION:}

Communication between neurons happens at specialized contact sites called synapses. Synapses contain a myriad of different proteins that orchestrate synaptic transmission. Some of those proteins show a heterogeneous distribution throughout the nervous system and are not present in every synapse ${ }^{1}$. One example for such a protein is Munc13, which is involved in the priming process of synaptic vesicles. There are different isoforms of Munc13, which are heterogeneously distributed throughout the brain ${ }^{2}$, and the presence or absence of specific isoforms can influence short-term synaptic plasticity and synaptic vesicle dynamics ${ }^{3-5}$. Therefore, it is of vital importance to be able to identify the presence of different synaptic proteins across brain areas.

The methods of choice for quantification of synaptic proteins - so far - are mass spectrometry and Western blotting, rather than immunohistochemistry ${ }^{6-9}$. In some cases, several methods are used to complement each other to assess both the quantity and the localization of specific proteins (i.e., Wilhelm et $a l .{ }^{10}$ ). The method we describe here allows for the localization and quantification of proteins of interest without the need of using any biochemical method, simply employing immunofluorescent stainings. Another advantage here is that the quantification can 
be done over areas much smaller and, therefore, more specific, than those achieved by other methods. However, one has to take into consideration that a reliable reference protein is needed to assess the distribution of the protein of interest.

Fluorescent staining by immunohistochemistry allows us to routinely identify the localization of proteins across brain areas as well as within different neuronal compartments. To identify the different compartments, specific markers are used. Typically, antibodies against synapsin and synaptophysin ${ }^{11}$ can be used to label synaptic vesicles, while antibodies against bassoon label the active zone of a presynaptic terminal ${ }^{12}$. Vesicular transporters, such as the vesicular glutamate transporters (vGluT) or vesicular GABA transporter (vGAT), are used to label excitatory $^{13}$ and inhibitory ${ }^{14}$ presynaptic terminals, respectively. On the postsynaptic side, antibodies against the Homer protein can be employed to mark postsynaptic terminals, and antibodies against postsynaptic density protein 95 (PSD95) $)^{15-17}$ or Gephyrin ${ }^{18-20}$ can label excitatory or inhibitory postsynaptic terminals, respectively. By using antibodies against a protein of interest and markers such as the ones described above, one can determine the localization of such protein. Many studies to date have done this in a qualitative manner ${ }^{21}$. However, to reliably determine the differential distribution of a specific synaptic protein, one must not only determine its presence or absence but also its relative concentration. The heterogeneity of sizes and density of synapses makes it important to establish a ratio between the synaptic marker and the protein of interest. Otherwise, synapse-rich regions such as the non-pyramidal layers of the hippocampus and the molecular layer of the cerebellum will show a high density of synaptic proteins, only due to the higher density of synapses but not due to a strong presence of that protein in each synapse (e.g., Wallrafen and Dresbach $\left.{ }^{1}\right)$. On the other hand, proteins in the neuronal soma (e.g., TGN38 $8^{22}$ ) will usually show strong presence in the hippocampal pyramidal cell layer or hippocampal or cerebellar granule cell layer due to the high concentration of neuronal cell bodies in those areas. Therefore, this non-homogeneous distribution of structures, in this case synapses, can lead to a false estimation of the distribution of the protein of interest itself. Furthermore, there is an intrinsic variability in staining intensities across samples in immunohistochemical stainings. The protocol described here takes this into consideration and avoids such biases, as well as other caveats that arise from immunohistochemical methods.

In our recent study, we have used this method to describe the differential expression of Mover (also called TPRGL ${ }^{23}$ or SVAP30 ${ }^{24}$ ) across 16 different brain areas ${ }^{1}$. Mover is a vertebrate-specific 
synaptic protein that can be found in association to synaptic vesicles and influences neurotransmitter release ${ }^{25-27}$. We have related the Mover expression to the abundance of synaptic vesicles, by staining for synaptophysin as a synaptic vesicle reference marker. We found high levels of Mover particularly in the septal nuclei, the ventral pallidum, and the amygdala. Within the hippocampus, we found a heterogeneous distribution of Mover, with high levels in the layers associated with intra-hippocampal computation, and low levels in input- and output layers.

\section{PROTOCOL:}

This protocol does not involve experiments on live animals. Experiments involving euthanizing of animals to obtain brain samples were approved by the local animal protection authorities (Tierschutzkommission der Universitätsmedizin Göttingen) under the approval number T 10/30.

NOTE: For this protocol, 3 adult male C57BL/6 mice were used.

\section{Sample Preparation}

1.1. Prepare fixative and $0.1 \mathrm{M}$ phosphate buffer (PB; see Table 1).

1.2. Fix the animal by transcardial perfusion as described in Gage et al. ${ }^{28}$. First wash out the blood with $0.9 \% \mathrm{NaCl}$-solution, then perfuse with $30 \mathrm{~mL}$ of $4 \%$ paraformaldehyde (PFA).

1.3. Open the skull with scissors and carefully isolate the brain using a spoon with blunt edges to avoid damaging the tissue.

1.4. Fill a $50 \mathrm{~mL}$ reaction tube with fixative and postfix the brain in $4 \%$ PFA at $4{ }^{\circ} \mathrm{C}$ overnight.

1.5. Remove the fixative and wash the brain in $50 \mathrm{~mL}$ of $0.1 \mathrm{M} \mathrm{PB}$ on a shaker for $30 \mathrm{~min}$.

1.6. After washing, incubate the brain in a $50 \mathrm{~mL}$ reaction tube in $30 \%$ sucrose in $0.1 \mathrm{M} \mathrm{PB}$ for 48 h or until it sinks in the tube at $4{ }^{\circ} \mathrm{C}$ for cryoprotection. 
1.7. Trim the cryoprotected brain with a sharp blade, place it in a cryomold, and embed it with optimal cutting temperature (OCT) compound. Avoid bubbles. Orient the brain and freeze the cryomold in the $-80^{\circ} \mathrm{C}$ freezer.

1.8. Mount the frozen tissue for sectioning. Equilibrate the tissue to the cryomicrotome temperature for at least 15 min before sectioning.

1.9. Section the brain into $25 \mu \mathrm{m}$ thick coronal slices. Touch the OCT carefully with a glass hook without touching the brain tissue. Collect 3 adjacent slices per well in a 24 well plate and store them in $0.1 \mathrm{M} \mathrm{PB}$ at $4{ }^{\circ} \mathrm{C}$ until staining.

NOTE: The protocol can be paused here for up to two weeks. Longer storage times can interfere with the tissue quality and thus influence the outcome of the experiment.

\section{Immunofluorescence}

2.1. Prepare solutions including the blocking buffer, antibody buffer, washing buffer 1 , and washing buffer 2 (see Table 1).

2.2. Rinse slices once with PB to remove excess OCT.

2.2.1. Remove the solution with a plastic pipette without sucking in the brain slices. Add $250 \mu \mathrm{L}$ of fresh PB with a $1000 \mu \mathrm{L}$ pipette.

CAUTION: Slices should not dry out, so remove and add fluids well by well.

2.3. Remove the PB with a plastic pipette and add $250 \mu \mathrm{L}$ of blocking buffer per well with a 1000 $\mu \mathrm{L}$ pipette. Incubate for $3 \mathrm{~h}$ at room temperature (RT) on the shaker.

2.4. During the incubation time, dilute the primary antibodies in antibody buffer in a reaction tube. Use $250 \mu \mathrm{L}$ antibody buffer per well and add the appropriate amount of antibody (see Table 2) by pipetting it directly into the solution using a $2 \mu \mathrm{L}$ pipette. Mix the solution by gently pipetting up and down several times. Vortex shortly afterwards to ensure proper mixing. 
NOTE: To determine the background fluorescence, stainings should also be performed without adding the primary antibody. For that, incubate the slice in antibody solution without primary antibodies according to the protocol.

2.5. After the incubation time, remove the blocking buffer with a plastic pipette and add $250 \mu \mathrm{L}$ of antibody solution containing primary antibodies per well. Incubate slices with primary antibody overnight at $4{ }^{\circ} \mathrm{C}$ on a shaker.

2.6. Next day, wash the slices with washing buffer $13 x$ for 10 min at RT on a shaker.

2.6.1. Remove the medium with a plastic pipette and add $300 \mu \mathrm{L}$ of washing buffer 1 per well. Incubate at RT for 10 min. Repeat 3 times.

2.7. During the washing steps, dilute the fluorophore-coupled secondary antibodies in antibody buffer in a reaction tube. Use $250 \mu \mathrm{L}$ antibody buffer per well and add the appropriate amount of antibody (see Table 2 ) by pipetting it directly into the solution using a $2 \mu \mathrm{L}$ pipette. Mix the solution by gently pipetting up and down several times. Vortex shortly afterwards to ensure proper mixing.

CAUTION: Because the antibodies are light-sensitive, all steps from this point on need to be performed in the dark.

2.8. After the washing steps, remove the washing buffer with a plastic pipette and add $250 \mu \mathrm{L}$ of antibody solution containing secondary antibodies per well. Incubate the slices with secondary antibody for 90 min at RT in the dark.

2.9. Wash the slices with washing buffer $23 x$ for $10 \mathrm{~min}$ at RT.

2.10. During the washing steps, dilute 4',6-diamidino-2-phenylindole (DAPI) in $0.1 \mathrm{M} \mathrm{PB}$ in a concentration of 1:2000.

2.11. Remove the washing buffer 2 with a plastic pipette and add $250 \mu \mathrm{L}$ of DAPI solution per well. Incubate for $5 \mathrm{~min}$ at RT on the shaker. 
2.12. Remove the DAPI solution with a plastic pipette and add $500 \mu \mathrm{L}$ of $0.1 \mathrm{M}$ PB per well with a $1000 \mu \mathrm{L}$ pipette.

2.13. Mount slices on microscope slides.

2.13.1. Place a microscope slide under the stereoscope. With a fine brush, add three separate drops of $0.1 \mathrm{M}$ PB onto the slide. Place one slice per drop onto the microscope slide.

2.13.2. Use the fine brush to flatten and orient the slices on the microscope slide.

2.13.3. When all slices are positioned correctly, remove excess PB with a tissue and dry the slide carefully.

CAUTION: Avoid drying the brain slices completely.

2.13.4. Add $80 \mu \mathrm{L}$ of embedding medium onto the slide. Carefully lower the coverslip onto the slide, thereby embedding the brain slices.

2.13.5. Leave the slides to dry in the fume hood for $1-2 \mathrm{~h}$ (cover them to avoid light exposure) and store them in a microscope slide box at $4{ }^{\circ} \mathrm{C}$.

NOTE: The protocol can be paused here.

\section{Imaging}

3.1. After the embedding medium is completely hardened, place the microscope slide under the confocal microscope.

NOTE: Epifluorescence microscopy combined with deconvolution software should yield similar image quality.

3.2. Adjust the laser settings by increasing or decreasing the laser intensity for every channel so that few pixels are overexposed to ensure maximum distribution of grey values.

3.3. Acquire virtual tissues of the whole brain slice for the different channels. 
3.3.1. In the imaging software (see Table of Materials), select the Tiles option and manually delineate the brain slice with the Tile Region Setup.

3.3.2. Distribute support points throughout the tile region and adjust the focus for the different support points by pressing Verify Tile Regions/Positions....

3.3.3. Adjust the settings in Acquisition Mode according to the desired resolution and file size of the resulting image and start the scan.

3.4. When the scan is finished, use the Stitching function to process the virtual tissue. Export the file as a .tif with the function Image Export.

\section{Computer-based Analysis}

4.1. Load all single channels for one image into $\mathrm{FIJI}^{29}$ by clicking File| Open.

4.2. With the Freehand selection tool, delineate one hemisphere in the DAPI-channel. Create a mask of the selection by clicking Edit| Selection | Create mask.

4.3. Determine the mean fluorescence intensity for the single channels (Mover and Synaptophysin) by clicking Analyze| Measure Particles.

NOTE: Make sure to select the different channels to determine the mean fluorescence intensity values for each channel.

4.4. Copy the mean fluorescence intensity for the single channels into a spreadsheet.

4.5. Determine the mean fluorescence intensity for the single channels in an area of interest by delineating the area also with the Freehand selection tool. Use a mouse brain atlas as reference.

4.6. Repeat steps 4.1-4.5 for all hemispheres and all areas of interest.

NOTE: Determine the values for each hemisphere separately in order to later compare the values in an area of interest to that in the hemisphere (see step 5.2). 


\section{Data Handling}

5.1. In case the background fluorescence is high (see Discussion), a background subtraction might be needed. For that, determine the mean fluorescence intensity for the slice processed without primary antibody against the reference protein (here: Synaptophysin) and subtract that value from all values obtained for the brain regions and hemispheres.

5.2. When the mean fluorescence intensities for the single channels for every hemisphere and every area of interest have been determined (see Table 3), calculate the ratio of Mover to Synaptophysin by dividing the value for Mover by the value for Synaptophysin (yellow in Table 3). Perform this action for every hemisphere and every area of interest separately.

5.3. Divide the ratio obtained for one area of interest by the ratio obtained for the corresponding hemisphere (orange in Table 3 ) to determine the ratio of the area of interest to the hemisphere.

5.4. To determine the relative Mover abundance, translate the ratio obtained in 5.2 into a percentage by determining its deviation from 1 (red in Table 3). A ratio of 1.25 would therefore give a relative Mover abundance of $25 \%$ above average, and a ratio of 0.75 would yield a relative Mover abundance of $25 \%$ below average.

\section{Fixative $(500 \mathrm{~mL})$ \\ Mix 20 g paraformaldehyde (total conc.: 4\%) \\ $50 \mathrm{~mL} 10 \mathrm{x}$ PBS stocksolution (total conc.: $1 \mathrm{x}$ ) \\ $450 \mathrm{~mL}$ bidest $\mathrm{H}_{2} \mathrm{O}$}

Adjust $\mathrm{pH}$ to 7.4 with $\mathrm{NaOH}$

Note: To solve the paraformaldehyde in PBS, heat the solution. Do not heat over $70^{\circ} \mathrm{C}$, as PFA disintegrates at temperatures higher than $70^{\circ} \mathrm{C}$.

Caution: PFA is toxic, potentially carcinogenic and teratogenic. Wear gloves when working with PFA and work under the fume hood. Avoid ingestion.

$0.1 \mathrm{M}$ PB (1 L)

Table1: Solutions used in this protocol 


\begin{tabular}{|l|l|}
\hline Stocksolution X & Stocksolution $\mathrm{Y}$ \\
$35.61 \mathrm{~g} \mathrm{Na}_{2} \mathrm{HPO}_{4} \bullet 2 \mathrm{H}_{2} \mathrm{O}$ in $1 \mathrm{~L}$ bidest $\mathrm{H}_{2} \mathrm{O}$ & $27.60 \mathrm{~g} \mathrm{NaH}_{2} \mathrm{PO}_{4} \bullet \mathrm{H}_{2} \mathrm{O}$ in $1 \mathrm{~L}$ bidest $\mathrm{H}_{2} \mathrm{O}$ \\
\hline Mix $\quad 385 \mathrm{~mL}$ stocksolution $\mathrm{X}$ \\
$115 \mathrm{~mL}$ stocksolution $\mathrm{Y}$ \\
$500 \mathrm{~mL}$ bidest $\mathrm{H}_{2} \mathrm{O}$
\end{tabular}

Table 2: Antibodies used in this protocol 


\begin{tabular}{|l|l|l|l|}
\hline \multicolumn{2}{|l|}{ Primary antibodies } \\
\hline Directed against & Host species & RRID & Concentration \\
\hline Mover & Rabbit & AB_10804285 & $1: 1000$ \\
\hline Synaptophysin & Guinea pig & AB_1210382 & $1: 1000$ \\
\hline Secondary antibodies & \multicolumn{3}{|l}{} \\
\hline Target species & Host species & Fluorophore & Concentration \\
\hline Rabbit & Donkey & AlexaFluor 647 & $1: 1000$ \\
\hline Guinea pig & Goat & AlexaFluor 488 & $1: 1000$ \\
\hline
\end{tabular}

Table 3: Example of data handling.

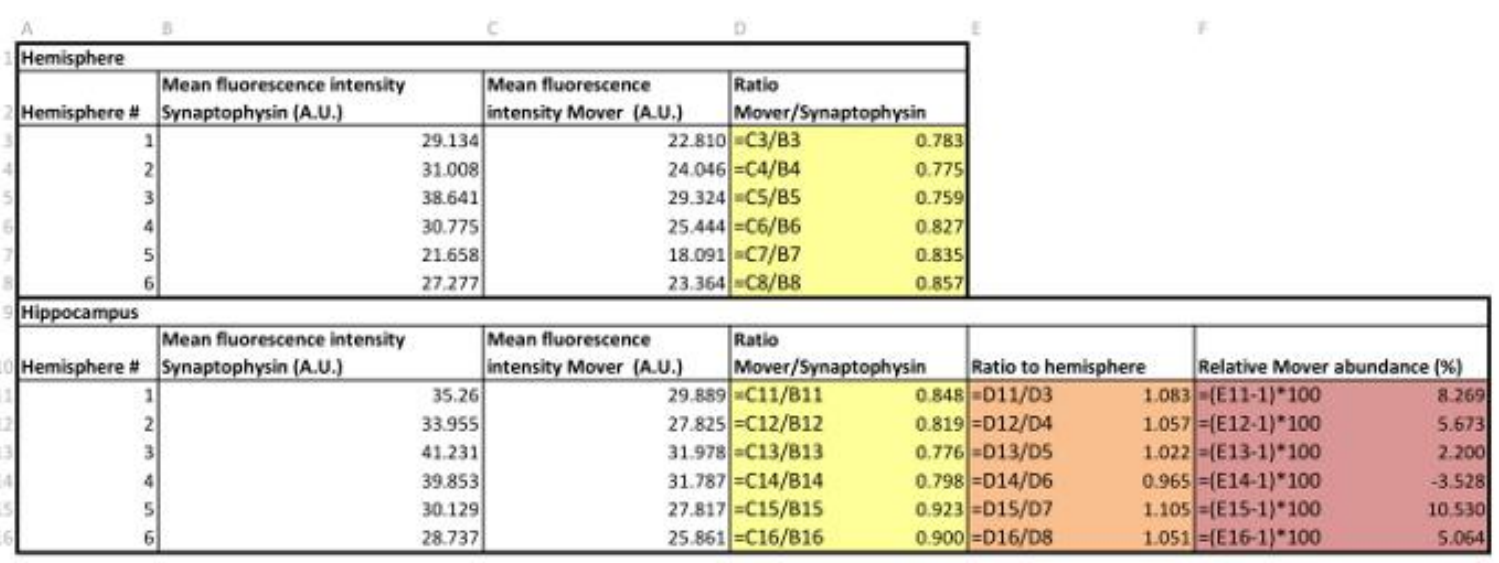

\section{REPRESENTATIVE RESULTS:}

Representative staining patterns of different markers can be seen in Figure 1. The pattern varies depending on the distribution of the protein. Examples of five rostro-caudal levels are shown in columns (A)-(E). A representative DAPI staining is shown in the first row: DAPI adheres to the DNA of a cell and thus nuclei are stained. This results in a punctate pattern. Regions with a high cell density are brighter than regions with low cell densities. An example for a heterogeneously distributed protein can be seen in the second row. The Mover staining reveals a differential distribution throughout the brain, with bright hotspot areas and dimmer areas. In the third row, an example for the more homogeneously distributed reference marker synaptophysin is shown. An overlay of the two proteins (fourth row) shows the differential distribution of Mover (red) compared to the marker protein Synaptophysin (green). 


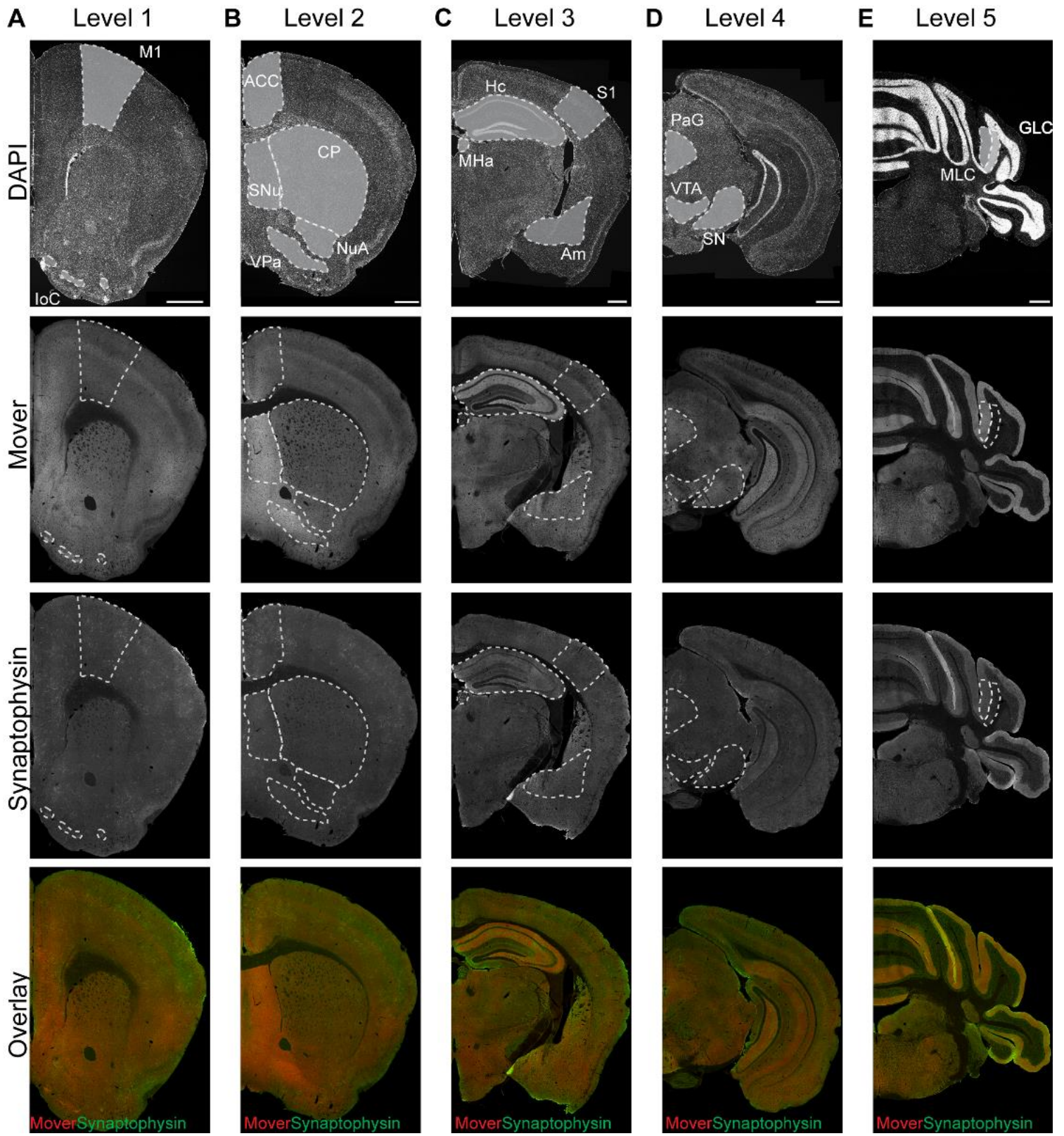

Figure 1: Representative immunofluorescence images of DAPI (first row), Mover (second row), synaptophysin (third row), and their overlay (fourth row, Mover in red, synaptophysin in green) at the 5 rostro-caudal levels (A-E). Areas of interest are shaded in grey in the upper row of panels. M1, primary motor cortex; IoC, islands of Calleja; $\mathrm{ACC}$, anterior cingulate cortex; $\mathrm{SNu}$, septal nuclei; VPa, ventral pallidum; NuA, nucleus accumbens; $\mathrm{CP}$, caudate putamen; S1, primary somatosensory cortex; $\mathrm{HC}$, hippocampus; Am, amygdala; MHa, medial habenula; PAG, periaqueductal grey; SN, substantia nigra; VTA, ventral tegmental area; MLC, molecular layer of the cerebellum; GLC, granular layer of the cerebellum. Scale bar $=500 \mu \mathrm{m}$. This figure has been modified from Wallrafen and Dresbach ${ }^{1}$.

Figure 2 shows the quantification described in step 4 of the protocol. Shown are the mean fluorescence intensity values for the different channels across the hemispheres (Mover, Figure 2A; Synaptophysin, Figure 2B) and across the areas of interest (Mover, Figure 2C; Synaptophysin, Figure 2D). To determine the Mover abundance relative to the number of synaptic vesicles, a ratio is taken of the Mover fluorescence values to Synaptophysin 
fluorescence values. These ratios for the areas of interest are shown in Figure 2E, and already provide an indication of the heterogeneous distribution of Mover, with areas with high and low Mover levels relative to synaptic vesicles. To additionally compensate for the inherent technical variability, the ratio in one area of interest (Figure 2E) is compared to that across the hemisphere (not shown) and translated into a percentage. This relative Mover abundance (Figure 2F) gives a measure of how much Mover is present in one area of interest relative to average.

As mentioned above, one of the major advantages of this technique is the ability to determine the abundance of the protein of interest across very small areas, even subregions and layers of areas of interest. One example of this application is shown in Figure 3, where the relative Mover abundance was determined for the different layers in the subfields of the hippocampus. The quantification in the different layers shown in Figure 3D, Figure $\mathbf{3 F}$, and Figure $\mathbf{3 H}$ corresponds to the layers shown in Figure 3C, Figure 3E, and Figure 3G, with the corresponding colors. Within the hippocampus, Mover is heterogeneously distributed, with high Mover levels relative to synaptic vesicles in layers associated with intra-hippocampal computation (i.e., the polymorph layer of dentate gyrus [DG], stratum radiatum, lucidum and oriens of Cornu Ammonis 3 [CA3], and stratum radiatum and oriens of Cornu Ammonis 1 [CA1]), and low levels in input- and output layers (the inner and outer molecular layer of DG, the pyramidal cell layers of CA3 and CA1, and the stratum lacunosum-moleculare of CA1). 

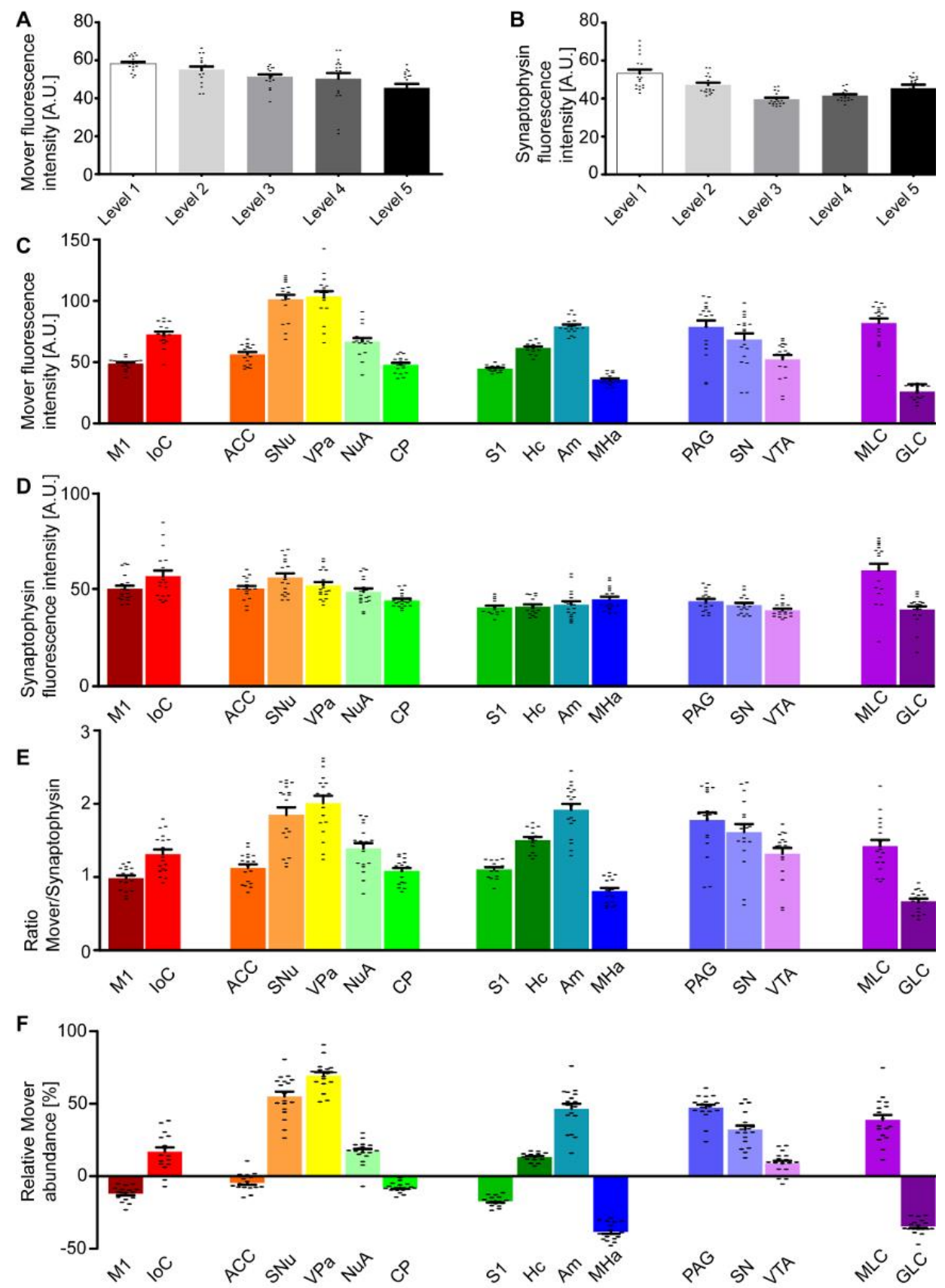

Figure 2: Quantification of the Mover distribution across the 5 rostro-caudal levels. Mean fluorescence intensity of the Mover signal (A) and the synaptophysin signal (B) at the different levels. Mean fluorescence intensity of the Mover signal (C) and the synaptophysin signal (D) at the 16 manually delineated brain regions. (E) Ratios of Mover and synaptophysin in the 16 brain areas of interest. (F) Quantification of the relative Mover abundance, comparing Mover/synaptophysin ratio at the respective region to the ratio of the corresponding hemisphere. $\mathrm{M} 1$, primary motor cortex; loC, islands of Calleja; ACC, anterior cingulate cortex; SNu, septal nuclei; VPa, ventral pallidum; NuA, nucleus accumbens; CP, caudate putamen; S1, primary somatosensory cortex; Hc, hippocampus; Am, amygdala; MHa, medial habenula; PAG, periaqueductal grey; SN, substantia nigra; VTA, ventral tegmental area; MLC, molecular layer of the cerebellum. Black dots represent single data points. Bars show the mean \pm standard error of the mean (SEM). This figure has been modified from Wallrafen and Dresbach ${ }^{1}$. 

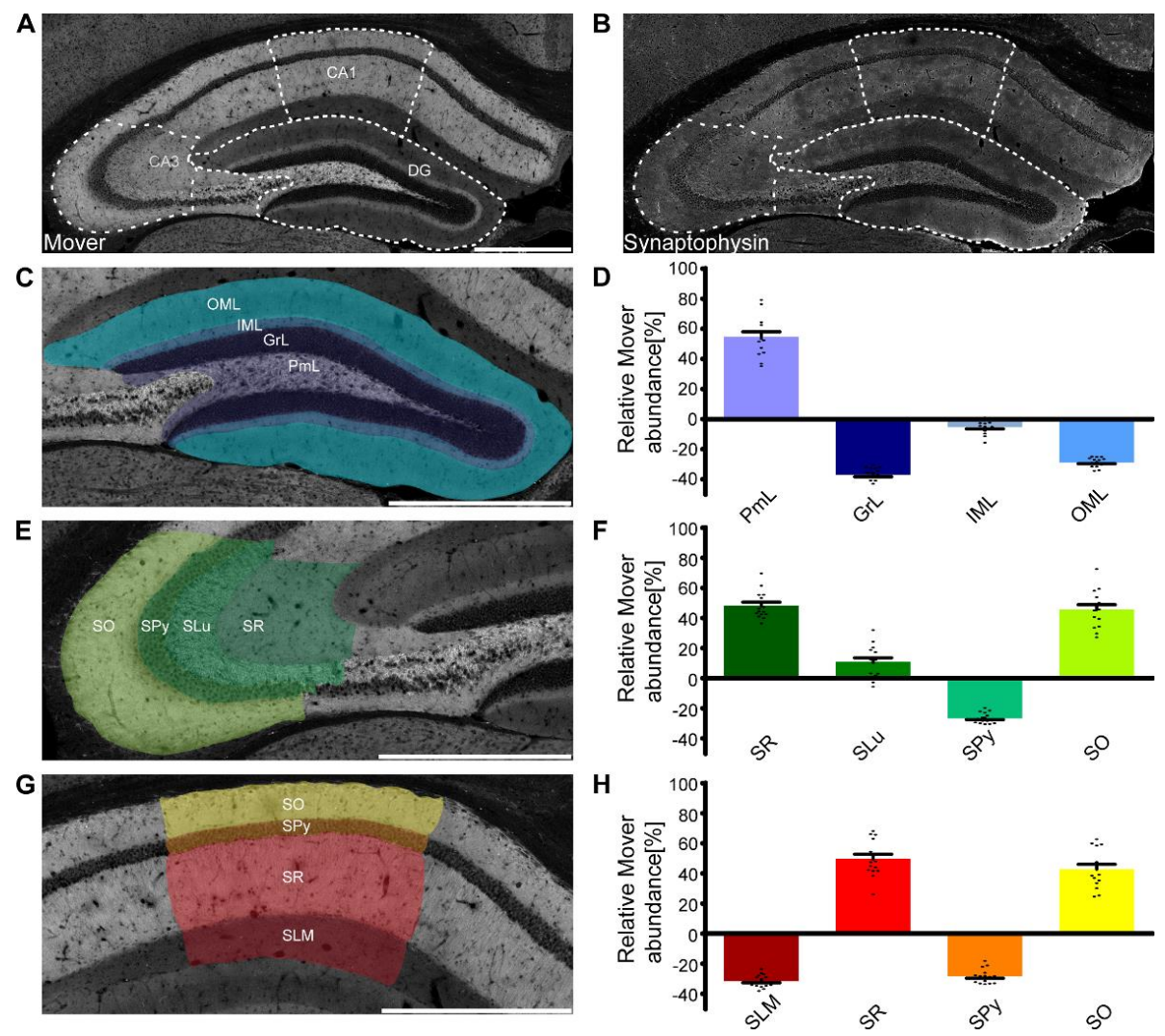

Figure 3: Mover distribution in the mouse hippocampus. Immunofluorescence stainings of coronal slices of the mouse hippocampus. Overview of the hippocampus showing the heterogeneous Mover expression pattern (A) and the corresponding Synaptophysin staining (B). The three regions of interest (DG, Figure 3C; CA3, Figure 3E; CA1, Figure 3G) are delineated with Fig. 3 (contd.): white dotted lines. (D,F,H) Quantification comparing the ratio in the respective layers to the ratio of the corresponding hemisphere. The colors in the bar graphs correspond to the respective shading in panels $\mathbf{C}, \mathbf{E}$, and $\mathbf{G}$. Mover expression is high in levels associated with intra-hippocampal computation (i.e., the polymorph layer of DG, stratum radiatum, lucidum and oriens of $C A 3$, and stratum radiatum and oriens of $C A 1$ ), and low in the main inputand output layers (the inner and outer molecular layer of DG, the pyramidal cell layers of CA3 and CA1, and the stratum lacunosum-moleculare of $\mathrm{CA} 1$ ). OML, outer molecular layer; IML, inner molecular layer; GrL, granular layer; PmL, polymorph layer/hilus; SO, stratum oriens; SPy, stratum pyramidale; SLu, stratum lucidum; SR, stratum radiatum; SLM, stratum lacunosum-moleculare. Scale bar $=500 \mu \mathrm{m}$. Black dots represent single data points. Bars show the mean \pm SEM. This figure has been modified from Wallrafen and Dresbach $^{1}$.

\section{DISCUSSION:}

The method presented here aims at quantifying the distribution of a protein of interest relative to the abundance of a marker protein with a known distribution. Immunofluorescence staining can show a high variability of staining intensities between different slices. The quantification approach described here circumvents this problem by determining the ratio of the protein of interest to the average across the hemisphere. Therefore, different staining intensities across slices are cancelled out and allow for a quantitative description.

As with every immunofluorescence protocol, qualitative or quantitative, several factors can influence the success and thereby confound the analysis. Therefore, special attention should be 
paid to critical steps of the protocol. First, a proper fixation of the tissue is needed. This fixation can usually be achieved by a successful transcardial perfusion. The quality of perfusion can be verified by checking the liver shortly after washing out the blood. A first indicator for a successful perfusion is the clearing of the liver and extremities ${ }^{28}$.

The presence of blood clots can indicate that the perfusion might have been too slow and should be performed faster next time. Some proteins require different fixation protocols, as chemical fixation with PFA can cause epitope blockage ${ }^{30}$. In this case, freeze fixation or fixation with a different chemical, such as methanol, should be considered. Second, after sectioning, it is critical to stain the brain slices as quickly as possible, preferably on the same or the next say. Longer storage in PB can lead to bacterial infection, and while adding $\mathrm{NaN}_{3}$ can prevent this to some extent, the tissue quality usually deteriorates with storage time. Third, during the staining procedure, it is important to perform washing steps well-by-well to avoid drying of the slices. When the slices dry out, background fluorescence can increase and thus cause a bias in the analysis. Fourth, after application of the secondary antibody, it is vital to perform all following steps in the dark. The fluorophores are light-sensitive, and light exposure can severely distort the fluorescence signal.

The optimization of the staining procedure, including the selection of adequate primary and secondary antibodies, optimal antibody concentration, and exposition time, is a prerequisite to achieve the best signal-to-noise ratio and to carry out a reliable quantitative immunofluorescence analysis. Antibodies verified in knock out tissue should be the preferred choice, albeit not always available. Always make sure to perform proper control experiments to exclude crosstalk between different antibodies. The amount of background fluorescence arising from autofluorescence and unspecific binding of the secondary antibody can be estimated by imaging the slices in which the primary antibody was not applied. It is not trivial to establish how much higher the intensities of the signal need to be when compared to the background to have an acceptable signal-to-noise ratio. However, based on empirical observations, the authors would suggest aiming for having a signal at least 2-fold stronger than background in order to reliably estimate the protein distribution. In case the background fluorescence is high in control conditions (without the presence of the primary antibody), the average background fluorescence should be subtracted from the experiment images.

The major advantage of our approach is its internal reference: the immunofluorescence intensity of the target protein (i.e., Mover) in a region of interest is compared to a reference marker (i.e., Synaptophysin) and to the overall intensity of these proteins across the entire hemisphere. Thus, from the calculation we perform, one can unequivocally conclude that the abundance of the 
target protein is $\mathrm{x}$ fold higher/lower in a certain region of interest than the abundance of the reference protein relative to the distribution of the proteins across the entire hemisphere at this level relative to Bregma ${ }^{31}$. This allows for the comparison of results using different antibodies, different microscopes, or even across different studies. This consistency across different samples comes from the comparative nature of this method: variability is compensated for by taking the ratio between the fluorescence in the area of interest and that of the hemisphere. Therefore, dissimilarities in absolute values arising from technical differences are nullified. Another major advantage of this technique is the fact that the areas of interest can be as small as you want them to be, only limited by the resolution of the microscope. Quantifying protein levels with biochemical methods, for example Western Blot or mass spectrometry ${ }^{6-9}$, requires a dissection of the tissue into the area of interest. This dissection is hard for regions of the brain, such as the primary somatosensory cortex, and becomes virtually impossible when aiming for subregions, such as the different layers of the cortex or the hippocampus.

A caveat of the approach is that the different levels in the brain cannot directly be compared with each other. Hemispheres with many regions rich in the protein of interest will have a different average value than hemispheres with only few protein-rich regions. Values of $20 \%$ above average, for example, will therefore reflect a different absolute quantity of protein in one level relative to Bregma as compared to a second one. One has to keep in mind as well that this method does not allow the determination of absolute protein levels, only the relative abundance compared to the internal reference marker and the average across the hemisphere.

This method can be easily adapted to determine the distribution of the protein of interest relative to markers for different neuronal compartments, not only presynaptic sites. It can also be easily adapted for tissues other than the brain and - with suitable antibodies - other model systems than mice ${ }^{32,33}$. While the use of a confocal microscope is the authors' method of choice, a combination of epifluorescence microscopy and deconvolution software should yield the same data quality and thus expand the usability of the protocol. Additionally, the same stainings can be used to determine the subcellular distribution, for example with super-resolution microscopy.

\section{DISCLOSURES:}

The authors have nothing to disclose.

\section{ACKNOWLEDGMENTS:}


We thank Irmgard Weiss for excellent technical assistance. The authors acknowledge support by Hermes Pofantis and Andoniya Petkova. The authors also thank the European Neuroscience Institute for the usage of the LSM800 and technical assistance, especially by Dr. Nils Halbsgut. This work was funded by the University Medical Center Göttingen. JSV acknowledges support by the Center for Nanoscale Microscopy and Molecular Physiology of the Brain (CNMPB).

\section{REFERENCES:}

1. Wallrafen, R., Dresbach, T. The Presynaptic Protein Mover Is Differentially Expressed Across Brain Areas and Synapse Types. Frontiers in Neuroanatomy 12, 58 (2018).

2. Augustin, I., Betz, A., Herrmann, C., Jo, T., Brose, N. Differential expression of two novel Munc13 proteins in rat brain. Biochemical Journal 337, 363-371 (1999).

3. Rosenmund, C. et al. Differential Control of Vesicle Priming and Short-Term Plasticity by Munc13 Isoforms. Neuron 33, 411-424 (2002).

4. Breustedt, J. et al. Munc13-2 differentially affects hippocampal synaptic transmission and plasticity. Cerebral cortex 20, 1109-20 (2010).

5. Chen, Z., Cooper, B., Kalla, S., Varoqueaux, F., Young, S. M. The Munc13 Proteins Differentially Regulate Readily Releasable Pool Dynamics and Calcium-Dependent Recovery at a Central Synapse. The Journal of Neuroscience 33, 8336-8351 (2013).

6. Taylor, S. C., Berkelman, T., Yadav, G., Hammond, M. A Defined Methodology for Reliable Quantification of Western Blot Data. (2013). doi:10.1007/s12033-013-9672-6

7. Charette, S. J., Lambert, H., Nadeau, P. J., Landry, J. Protein quantification by chemiluminescent Western blotting: Elimination of the antibody factor by dilution series and calibration curve. Journal of Immunological Methods 353, 148-150 (2010).

8. Heidebrecht, F., Heidebrecht, A., Schulz, I., Behrens, S., Bader, A. Improved semiquantitative Western blot technique with increased quantification range. Journal of Immunological Methods 345, 40-48 (2009).

9. Toki, M. I., Cecchi, F., Hembrough, T., Syrigos, K. N., Rimm, D. L. Proof of the quantitative potential of immunofluorescence by mass spectrometry. Laboratory Investigation 97, 329-334 (2017).

10. Wilhelm, B. G. et al. Composition of isolated synaptic boutons reveals the amounts of vesicle trafficking proteins. Science 344, 1023-1028 (2014).

11. Navone, F. et al. Protein p38: An integral membrane protein specific for small vesicles of neurons and neuroendocrine cells. Journal of Cell Biology 103, 2511-2527 (1986).

12. Gundelfinger, E. D., Reissner, C., Garner, C. C. Role of Bassoon and Piccolo in Assembly 
and Molecular Organization of the Active Zone. Frontiers in Synaptic Neuroscience 7, (2016).

13. Ziegler, D. R., Cullinan, W. E., Herman, J. P. Distribution of vesicular glutamate transporter mRNA in rat hypothalamus. Journal of Comparative Neurology 448, 217-229 (2002).

14. Chaudhry, F. A. et al. The vesicular GABA transporter, VGAT, localizes to synaptic vesicles in sets of glycinergic as well as GABAergic neurons. Journal of Neuroscience 18, 97339750 (1998).

15. Aoki, C. et al. Electron Microscopic Immunocytochemical SAP-97 at Postsynaptic, Presynaptic, and Nonsynaptic Sites of Adult and Neonatal Rat Visual Cortex. Synapse 257, 239-257 (2001).

16. Valtschanoff, J. G. et al. Expression of NR2 Receptor Subunit in Rat Somatic Sensory Cortex: Synaptic Distribution and Colocalization With NR1 and PSD-95. Journal of Comparative Neurology 611, 599-611 (1999).

17. Hunt, A., Schenker, L. J., Kennedy, B. PSD-95 Is Associated with the Postsynaptic Density and Not with the Presynaptic Membrane at Forebrain Synapses. Journal of Neuroscience 76, 1380-1388 (1996).

18. Luscher, B., Fuchs, T., Kilpatrick, C. L. GABA A Receptor Trafficking-Mediated Plasticity of Inhibitory Synapses. Neuron 70, 385-409 (2011).

19. Kirby, M. Understanding the molecular diversity of GABAergic synapses. 5, 1-12 (2011).

20. Harris, K. M., Weinberg, R. J., Verrall, A. W. Ultrastructure of Synapses in the Mammalian Brain. Cold Spring Harbor Perspectives in Biology 1-30 (2012).

21. Heise, C. et al. Selective Localization of Shanks to VGLUT1-Positive Excitatory Synapses in the Mouse Hippocampus. Frontiers in Cellular Neuroscience 10, (2016).

22. Peters, P. J., Yuan, L. C., Biology, C., Branch, M. Localization of TGN38 to the trans-Golgi network: involvement of a cytoplasmic tyrosine-containing sequence. Journal of Cell Biology 120, 1123-1135 (1993).

23. Antonini, D. et al. Tprg, a gene predominantly expressed in skin, is a direct target of the transcription factor p63. Journal of Investigative Dermatology 128, 1676-1685 (2008).

24. Burré;, J. et al. Synaptic vesicle proteins under conditions of rest and activation: Analysis by 2-D difference gel electrophoresis. Electrophoresis 27, 3488-3496 (2006).

25. Kremer, T. et al. Mover is a novel vertebrate-specific presynaptic protein with differential distribution at subsets of CNS synapses. FEBS Letters 581, 4727-4733 (2007).

26. Ahmed, S. et al. Mover Is a Homomeric Phospho-Protein Present on Synaptic Vesicles. 
PLOS ONE 8, (2013).

27. Körber, C. et al. Modulation of Presynaptic Release Probability by the VertebrateSpecific Protein Mover. Neuron 87, 521-533 (2015).

28. Gage, G. J., Kipke, D. R., Shain, W. Whole Animal Perfusion Fixation for Rodents. Journal of Visualized Experiments 1-9 (2012). doi:10.3791/3564

29. Schindelin, J. et al. Fiji: an open-source platform for biological-image analysis. Nature Methods 9, (2012).

30. Schneider Gasser, E. M. et al. Immunofluorescence in brain sections: simultaneous detection of presynaptic and postsynaptic proteins in identified neurons. Nature Protocols 1, 1887-1897 (2006).

31. Paxinos, G., Franklin, K. B. J. The Mouse Brain in Stereotaxic Coordinates. (ACADEMIC PRESS, 2001).

32. Sun, Y., Ip, P., Chakrabartty, A. Simple Elimination of Background Fluorescence in Formalin-Fixed Human Brain Tissue for Immunofluorescence Microscopy. Journal of Visualized Experiments 1-6 (2017).

33. Poole, A. R., Dingle, J. T., Mallia, A. K. The localization of retinol-binding protein in rat liver by immunofluorescence microscopy. Journal of Cell Science 394, 379-394 (1975). 
Wallrafen, 2019 


\section{THE PRESYNAPTIC PROTEIN}

MOVER IS HETEROGENEOUSLY

\section{DISTRIBUTED ACROSS BRAIN AREAS}

\section{AND SYNAPSES TYPES}

Frontiers in Neuroanatomy, published $13^{\text {th }}$ of July 2018

Doi: 10.3389/fnana.2018.00058

\section{ABSTRACT}

"The assembly and function of presynaptic nerve terminals relies on evolutionarily conserved proteins. A small number of presynaptic proteins occurs only in vertebrates. These proteins may add specialized functions to certain synapses, thus increasing synaptic heterogeneity. Here, we show that the vertebrate-specific synaptic vesicle (SV) protein mover is differentially distributed in the forebrain and cerebellum of the adult mouse. Using a quantitative immunofluorescence approach, we compare the expression of mover to the expression of the general SV marker synaptophysin in 16 brain areas. We find that mover is particularly abundant in the septal nuclei (SNu), ventral pallidum (VPa), amygdala and hippocampus. Within the hippocampus, mover is predominantly associated with excitatory synapses. Its levels are low in layers that receive afferent input from the entorhinal cortex, and high in layers harboring intra- hippocampal circuits. In contrast, mover levels are high in all nuclei of the amygdala, and mover is associated with inhibitory synapses in the medioposterior amygdala. Our data reveal a striking heterogeneity in the abundance of mover on three levels, i.e., between brain areas, within individual brain areas and between synapse types. This distribution suggests a role for mover in providing specialization to subsets of synapses, thereby contributing to the functional diversity of brain areas." (Wallrafen \& Dresbach, 2018) 


\section{Authors}

Rebecca Wallrafen, Thomas Dresbach*

Synaptogenesis group, Institute of Anatomy and Embryology, University Medical Center Goettingen, Goettingen, Germany

\section{*Correspondence:}

Corresponding Author

thomas.dresbach@med.uni-goettingen.de

Keywords: mover, synaptic protein, distribution, immunofluorescence, quantification, hippocampus, amygdala

\section{Introduction}

Neurotransmitter release is mediated by a molecular machinery consisting of proteins mediating synaptic vesicle anchoring, priming and fusion at specialized sites of presynaptic nerve terminals called active zones (Fejtova \& Gundelfinger, 2006; Südhof, 2012). The vast majority of presynaptic proteins is evolutionarily conserved, but a remarkably small number of proteins is unique to vertebrates. These include the active zone scaffolding proteins bassoon and piccolo, the motor adaptor syntabulin, and the synaptic vesicle (SV) proteins synuclein and mover (Cai et al., 2007; George, 2002; Gundelfinger et al., 2016; Kremer et al., 2007). It has been suggested that the major role of vertebrate-specific synaptic proteins is to increase the functional heterogeneity of synapses in the brain (Emes et al., 2008; Ryan \& Grant, 2009). Numerous examples have demonstrated heterogeneous release probability and short-term plasticity among synapses in the neocortex, hippocampus and cerebellum (Blackman et al., 2013). While some of this heterogeneity may arise from different combinations of isoforms and posttranslational modifications of the conserved core machinery, vertebrate-specific proteins may add additional versatility to this machinery. In particular, cell- or synapse-specific expression of such proteins may endow certain synapses with special features.

Bassoon is a vertebrate-specific component of active zones that is found at all synapses and may generally stabilize presynaptic boutons by reducing proteasomal degradation (Okerlund et al., 2017; Waites et al., 2013). Bassoon interacts with - among a number of evolutionarily conserved proteins - two of the vertebrate-specific proteins, i.e. syntabulin (Cai et al., 2007) and mover (Thomas Kremer et al., 2007). We had identified mover as a binding partner for bassoon in a 
yeast-2-hybrid assay and found that it is a phosphoprotein of SVs (Ahmed et al., 2013; Kremer et al., 2007). Knockdown of mover at the calyx of Held synapse resulted in increased release probability and short-term depression, suggesting that mover regulates synaptic strength and plasticity at this synapse (Körber et al., 2015). Mover has also been detected in a proteomic analysis of SV fractions, where it was called SVAP-30 (Burré et al., 2006). In addition, mover is called TPRGL. It appears to have co-evolved with a similar protein called TPRG by gene duplication in vertebrates, and mover/TPRGL and TPRG are each located next to a vertebratespecific transcription factor, called p73 and p63, respectively (Antonini, Dentice, Mahtani, De Rosa, et al., 2008).

Interestingly, unlike bassoon, mover appeared to be absent from some synapses: on a qualitative level, we had detected mover in hippocampal mossy fiber terminals and at the calyx of Held, i.e. two glutamatergic synapses, and at inhibitory synapses in the cerebellum. In contrast, we had not detected mover at inhibitory terminals in the stratum lucidum of the hippocampus (Thomas Kremer et al., 2007). To test whether mover is indeed a candidate protein that could increase the functional heterogeneity of synapses we tested its distribution on a quantitative level.

\section{Material and Methods}

\section{Experimental animals}

No experiments involving live animals were conducted for this study. Experiments involving euthanizing of animals to obtain brain samples were approved by the local animal protection authorities (Tierschutzkommission der Universitätsmedizin Göttingen) under the approval number T 10/30.

\section{Immunofluorescence staining}

For this study, three adult male wild-type (wt) $\mathrm{C} 57 \mathrm{BL} / 6$ mice were euthanized by $\mathrm{CO}_{2}$ inhalation and transcardially perfused with $0.9 \%$ saline followed by perfusion with $4 \%$ paraformaldehyde (PFA) in $0.1 \mathrm{M}$ phosphate buffer (PB), pH 7.4. Brains were removed and postfixed in 4\% PFA in $0.1 \mathrm{M} P B$ for 24 hours at $4^{\circ} \mathrm{C}$ and incubated in $30 \%$ sucrose at $4^{\circ} \mathrm{C}$ for 48 hours.

Brains were cut into $25 \mu \mathrm{m}$ thick coronal sections using a freezing microtome. Sections were collected in $0.1 \mathrm{M} \mathrm{PB}$ and stored at $4^{\circ} \mathrm{C}$ until further use. Five positions relative to bregma were selected according to the mouse brain atlas (Paxinos \& Franklin, 2001), and per level, three adjacent slices per brain were stained (bregma ranges: $+1.5 \mathrm{~mm},+1.0 \mathrm{~mm},-2.0 \mathrm{~mm},-3.5 \mathrm{~mm},-$ $6.0 \mathrm{~mm}$ ). Free floating sections were rinsed with PB once and blocked with $2.5 \%$ goat serum 
(Merck KGaA, Darmstadt, Germany), 2.5\% donkey serum (Merck Chemikals GmbH, Darmstadt, Germany), $1 \%$ Triton X-100 in PB for 3 hours at room temperature (RT). The following relevant primary antibodies were applied overnight at $4^{\circ} \mathrm{C}$ : mover (1:1000 rabbit anti-mover polyclonal, Cat. No. 248003, RRID: AB_10804285, Synaptic Systems, Goettingen, Germany), synaptophysin (1:1000 guinea pig anti-synaptophysin polyclonal, Cat. No. 101004, RRID: AB_1210382, Synaptic Systems, Goettingen, Germany), vGlut1 (1:1000 guinea pig anti vGluT1 polyclonal, Cat. No. 135304, RRID: AB_887878, Synaptic Systems, Goettingen, Germany) and vGAT (1:500 chicken anti-vGAT polyclonal, Cat. No. 131006, RRID: AB_2619820, Synaptic Systems, Goettingen, Germany). Sections were washed with $2 \%$ goat serum in PB and incubated with relevant secondary antibodies for $90 \mathrm{~min}$ at RT in the dark: donkey anti-rabbit 647 (1:1000, Alexa Fluor, Invitrogen, Carlsbad, CA, USA), goat anti-guinea pig Cy2, goat anti-chicken Cy3 (1:1000, Jackson ImmunoResearch, West Grove, PA, USA; all antibodies were diluted in $0.5 \%$ goat serum, $0.5 \%$ donkey serum, $0.2 \%$ Triton $\mathrm{X}-100$ in $\mathrm{PB}$ ). Sections were washed with $1 \%$ goat serum in $\mathrm{PB}$, incubated with 4',6-diamidino-2-phenylindole (DAPI; 1:1000 in PB) for $5 \mathrm{~min}$, rinsed again and mounted on Menzel microscope slides. To ensure minimal variability, brains from all animals were cut, stained and treated simultaneously.

\section{Microscopy}

All images were acquired using a Zeiss LSM800 confocal microscope, running the ZEN blue software (version 2.3, Zeiss, Oberkochen, Germany). Laser settings were adjusted so that few pixels were overexposed to ensure maximum distribution of grey values.

Distribution analysis To analyze the distribution of mover throughout the brain, double stainings for mover and synaptophysin were performed and virtual tissues composed of single tiles (1024x1024 px, moderate scan speed, 4-times averaging) were acquired using a 10x objective (air immersion, NA 0.45). The whole brain slice was imaged. Using the corresponding functions of the program, virtual tissues were stitched and exported as TIFF-files.

Colocalization analysis To determine the colocalization of mover with vGluT1 and vGAT, triple stainings were performed and single pictures in the ROI were acquired using a 40x objective (oil immersion, NA 1.3). No adjustments for brightness or contrast were made, and images were exported as TIFF-files.

\section{Quantification}

For quantification, areas of interest were delineated manually using FIJI (ImageJ v.1.51r) with the mouse brain atlas as reference. Mean fluorescence intensity values were determined for one area of interest for the synaptophysin and mover channels. These values were transferred to 
Microsoft Excel for data handling. To determine their ratio, the value from the mover channel was divided by the value from the synaptophysin channel. The ratio in one area of interest was then compared to the ratio in the corresponding hemisphere. We performed these actions for every brain region and slice separately, i.e. always comparing the ratio of mover versus synaptophysin in the area of interest to the ratio of mover versus synaptophysin in the hemisphere on the same slice. If the ratio in one brain region of interest and in the hemisphere were the same, the resulting ratio would be 1 . Next, we determined how much the ratio in one brain area differs from the ratio in the hemisphere. We calculated the percentage by which the ratio of mover versus synaptophysin in the area of interest to the ratio of mover versus synaptophysin in the hemisphere differed from 1 (e.g. an overall ratio of 0.95 would be $5 \%$ below average, an overall ratio of 1.25 would be $25 \%$ above average). We refer to this percentage as the relative abundance of mover. For visualization, values are displayed in a scatter plot including bar charts (indicating average \pm S.E.M., GraphPrism 6).

\section{Colocalization analysis}

To analyze the colocalization of mover with vGluT1 and vGAT, respectively, we subtracted the background staining from the monochromatic image using the "Subtract Background" function of FIJI (Rolling ball radius: 100 pixels for all channels). We then used the "Colocalization Test" plugin to determine the Pearson's correlation coefficient between mover and vGluT1, between mover and vGAT and vGluT1 and VGAT as a means of control (for more information on Pearson's correlation see Adler \& Parmryd, 2010 and Dunn, Kamocka, \& McDonald, 2011). To verify that no random colocalization was measured, we rotated one of the images by $90^{\circ}$ and analyzed colocalization: doing this, no colocalization was observed, and Pearson's correlation values were very low to negative. Values were plotted in scatter plots including bar charts (average \pm S.E.M) and a Student's t-test was performed using GraphPrism 6.

\section{Antibody specificity}

To verify the absence of non-specific immunostaining using the immunofluorescence method, primary antibodies were excluded but the secondary antibody steps were performed to completion. Under these conditions, no cross-reactivity or significant background staining was observed (not shown).

\section{Results}

Mover is heterogeneously expressed throughout the adult mouse brain 

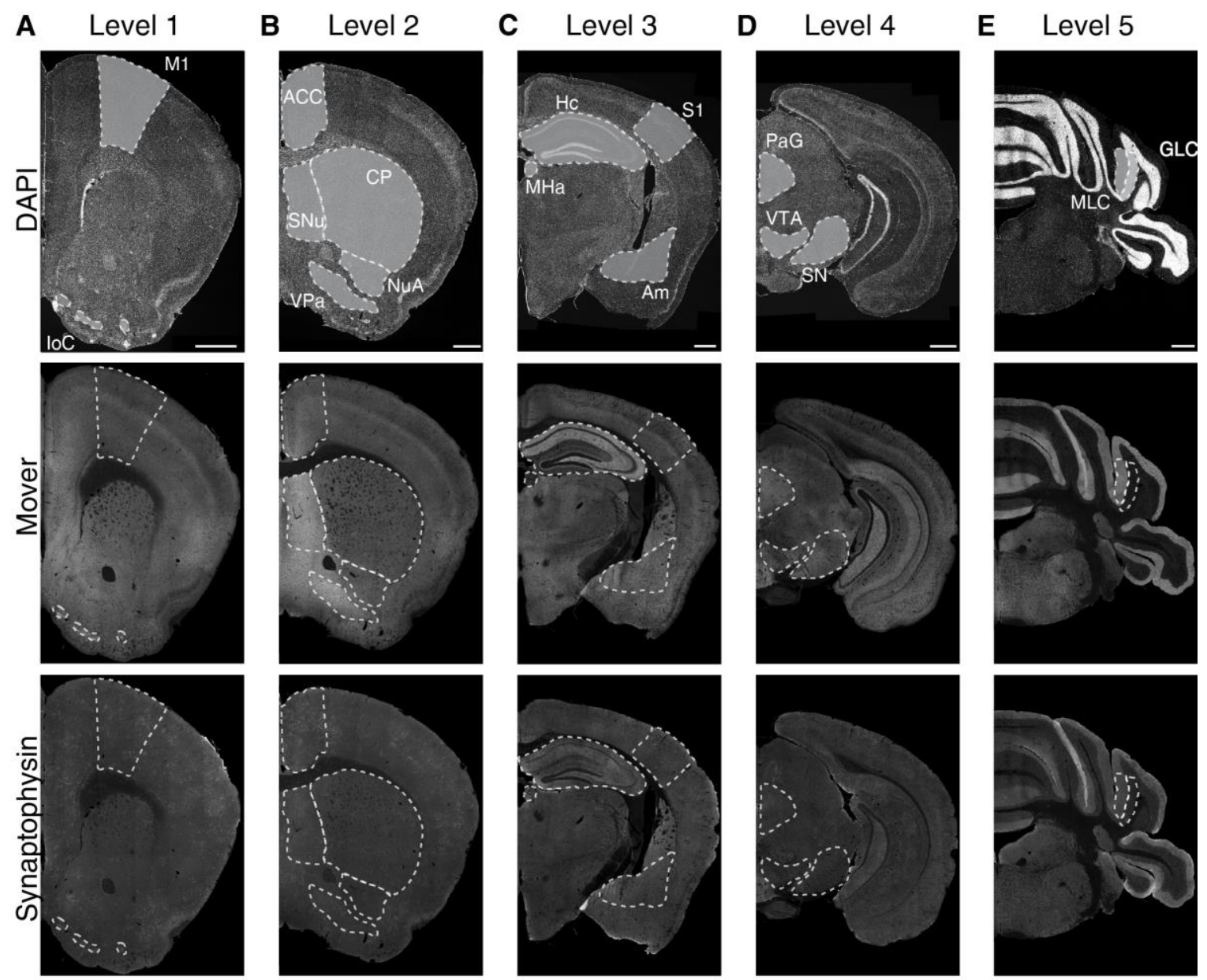

To determine the regional distribution of the synaptic protein mover, we performed immunofluorescence double-stainings on 5 rostro-caudal levels for mover and synaptophysin, an integral membrane protein of synaptic vesicles in all synapses (Navone et al., 1986), and counterstained the slices with DAPI (Figure 1, upper panels). Upon inspection, mover seemed

heterogeneously distributed (Figure 1, middle panels), with areas of obviously high signal intensity, while synaptophysin signals were fairly constant across all areas of the hemispheres

Figure 1: Immunofluorescence images of DAPI, mover and synaptophysin at the 5 rostro-caudal levels. We examined 5 coronal levels of the mouse brain (Level 1-5, A-E) and manually delineated 16 brain regions of interest (delineated with white dotted lines). The upper panels show the DAPI counterstaining, displaying the general anatomy at the plane of sectioning. Note the heterogeneous distribution of mover throughout the levels (middle panels), while the distribution of synaptophysin (lower panels) is rather homogeneous. M1, primary motor cortex; loC, islands of Calleja; ACC, anterior cingulate cortex; SNu, septal nuclei; $\mathrm{VPa}$, ventral pallidum; NuA, nucleus accumbens; CP, caudate putamen; S1, primary somatosensory cortex; $\mathrm{Hc}$, hippocampus; Am, amygdala; $\mathrm{MHa}$, medial habenula; PAG, periaqueductal grey; SN, substantia nigra; VTA, ventral tegmental area; MLC, molecular layer of the cerebellum; GLC, granular layer of the cerebellum. Scale bar $=500 \mu \mathrm{m}$. 
(Figure 1, lower panels). We selected 16 brain regions for quantification of mover: primary motor cortex (M1), islands of Calleja (loC), anterior cingulate cortex (ACC), septal nuclei (SNu), ventral pallidum ( $\mathrm{VPa})$, nucleus accumbens ( $\mathrm{NuA})$, caudate and putamen $(\mathrm{CP})$, primary somatosensory cortex (S1), hippocampus ( $\mathrm{Hc}$ ), amygdala (Am), medial habenula (MHa), periaqueductal grey (PAG), substantia nigra (SN), ventral tegmental area (VTA), molecular layer of the cerebellum (MLC) and granular layer of the cerebellum (GLC). Mover immunofluorescence intensities were particularly high in the septal nuclei, the ventral pallidum and the amygdala, and strikingly low in the granular layer of the cerebellum, the medial habenula and the primary somatosensory cortex.

The difference in mover immunofluorescence intensities between the areas could reflect either of two scenarios: a) areas with increased mover immunofluorescence intensities have synapses with an increased concentration of mover per SV; b) areas with increased mover immunofluorescence intensities have synapses with more SVs. To test whether some synapses have a higher concentration of mover than others, we set out to quantify our observation. To this end, a marker representing the number of SVs per synapse has to be introduced. In our study, the integral SV protein synaptophysin represents this parameter. We first determine the meain fluorescence intensity (MFI) across the whole hemisphere for the two different channels, i.e. mover (Figure 2A) and synaptophysin (Figure 2B).

For every hemisphere, we calculated a ratio, i.e. $\mathrm{MFI}_{\text {mover }}$ divided by $\mathrm{MFI}_{\text {synaptophysin, }}$ at each of the 5 rostro-caudal levels. This ratio represents the abundance of mover across the entire hemisphere for a given level. In addition, from every raw data point shown in Figure $\mathbf{2 C}$ and $\mathbf{D}$, we calculated a ratio, i. e. $\mathrm{MFI}_{\text {mover }}$ divided by $\mathrm{MFI}_{\text {synaptophysin }}$ for the 16 manually delineated brain areas. Next, we divided the ratio of mover to synaptophysin calculated in one brain region by their ratio calculated for the corresponding hemisphere.

The resulting value represents the abundance of mover within that brain region compared to its abundance across the hemisphere, and corrects for the number of SVs per synapse by considering the intensity of synaptophysin staining (Figure 2E). The value exceeds 1 if the ratio of mover versus synaptophysin is increased in a certain brain area compared to the overall ratio obtained for the hemisphere; in contrast, it is lower than 1 if the ratio of mover versus synaptophysin is decreased in a certain brain area compared to the overall ratio obtained for the hemisphere. We calculated the difference from 1 in percent, and refer to this value as the as the relative abundance of mover. Importantly, this procedure corrects for variabilities in staining intensities between sections, and even between mover and synaptophysin: if one specific staining for synaptophysin was weaker or stronger than usual, this change would still be 
observed on the entire hemisphere, and the relative abundance of mover in an area of interest could still be determined. 
A

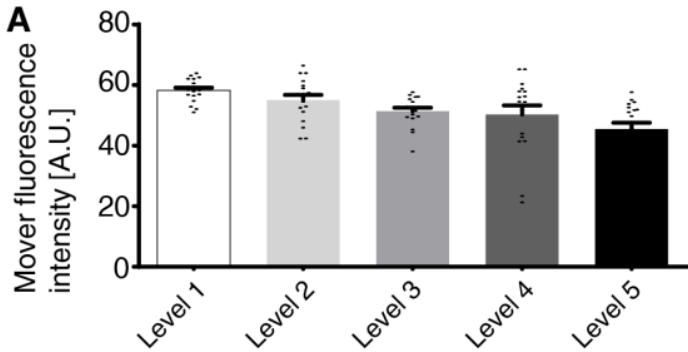

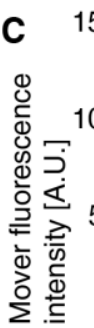

B

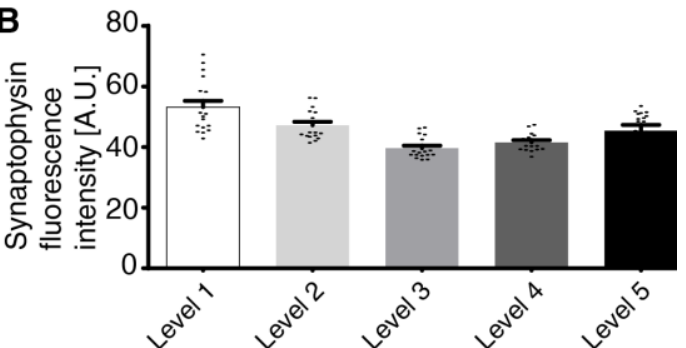

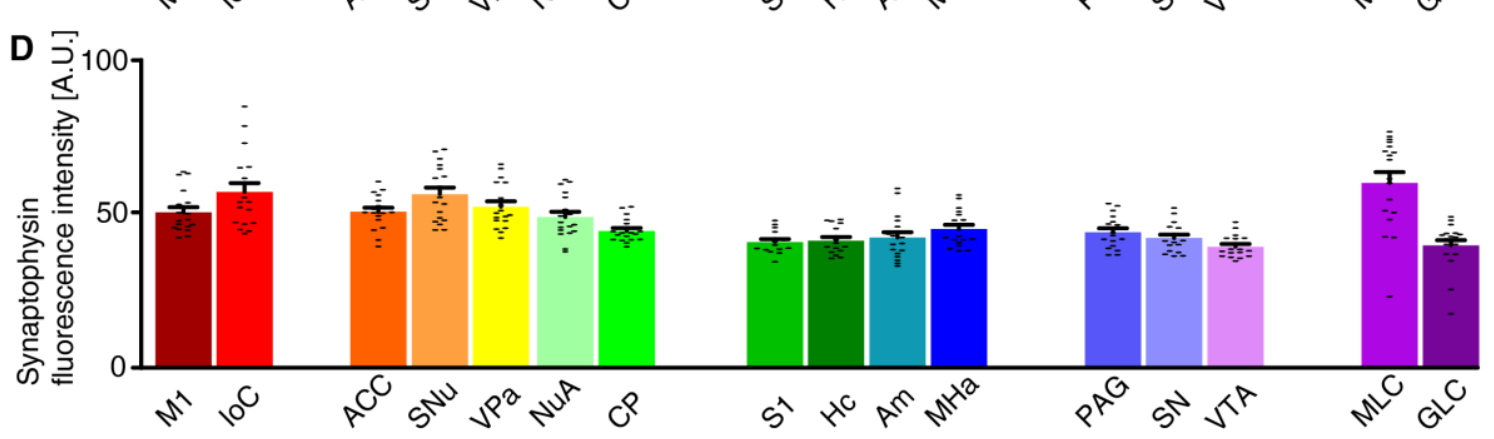

E

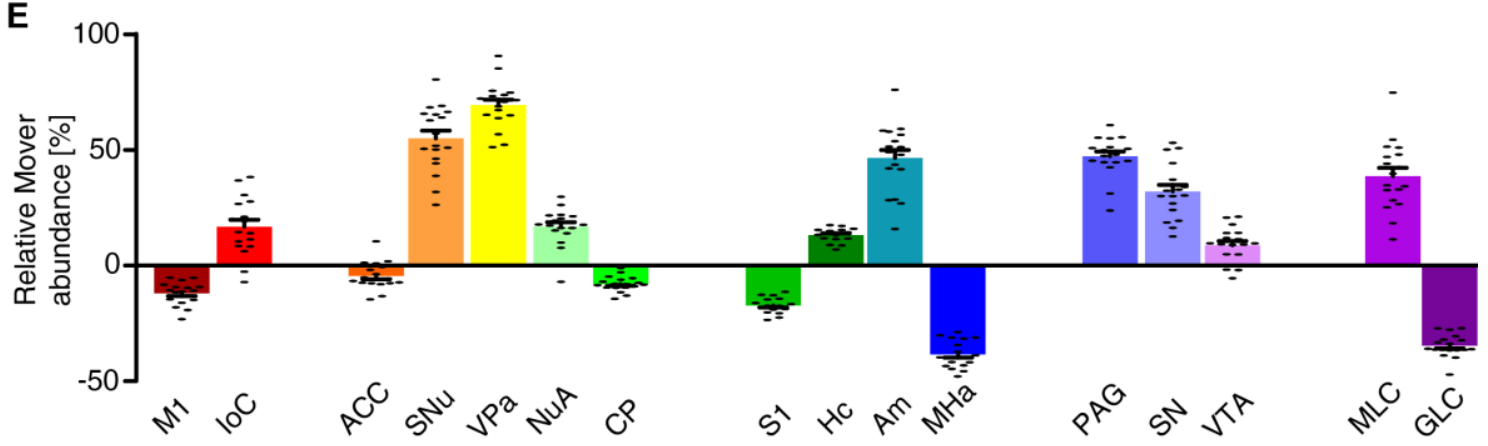

Figure 2: Quantification of the mover distribution across the 5 rostro-caudal levels. (A,B) Mean fluorescence intensity of the mover signal $(A)$ and the synaptophysin signal $(B)$ at the different levels. $(C, D)$ Mean fluorescence intensity of the mover signal (C) and the synaptophysin signal (D) at the 16 manually delineated brain regions. (E) Quantification comparing mover/synaptophysin ratio at the respective region to the ratio of the whole hemisphere. Mover fluorescence is above average in islands of Calleja, but below average in M1. Mover levels are above average in the ventral pallidum and nucleus accumbens and below average in the anterior cingulate cortex and caudate and putamen. Mover levels are above average in the hippocampus and amygdala and below average in S1 and the medial habenula. Mover levels are above average in the periaqueductal grey, substantia nigra and ventral tegmental area. Mover levels are above average in the molecular layer of the cerebellum and below average in the granular layer of cerebellum. M1, primary motor cortex; IoC, islands of Calleja; ACC, anterior cingulate cortex; SNu, septal nuclei; VPa, ventral pallidum; NuA, nucleus accumbens; $\mathrm{CP}$, caudate putamen; S1, primary somatosensory cortex; $\mathrm{Hc}$, hippocampus; Am, amygdala; MHa, medial habenula; PAG, periaqueductal grey; SN, substantia nigra; VTA, ventral tegmental area; MLC, molecular layer of the cerebellum; GLC, granular layer of the cerebellum. 
At bregma $+1.5 \mathrm{~mm}$, the relative abundance of mover was above average of the hemisphere in islands of Calleja (16.89 $\pm 2.83 \%)$, and below average of the hemisphere in primary motor cortex, where it was heterogeneously distributed throughout the layers $(-12.03 \pm 1.12 \%)$. At bregma $+1.0 \mathrm{~mm}$, mover abundance was above average in septal nuclei, ventral pallidum and nucleus accumbens $(55.02 \pm 3.25 \%, 69.45 \pm 2.28 \%$ and $16.91 \pm 1.81 \%$ respectively), and below average in anterior cingulate cortex and caudate putamen $(-4.51 \pm 1.34 \%$ and $-7.99 \pm 0.75 \%)$. More caudally, at bregma $-2.0 \mathrm{~mm}$, high mover levels were detected in hippocampus and amygdala $(13.13 \pm 0.79 \%$ and $46.57 \pm 3.31 \%$ ), while low levels were detected in primary somatosensory cortex and medial habenula $(-17.26 \pm 0.85 \%$ and $-38.41 \pm 1.35 \%)$. In the primary somatosensory cortex, like in the primary motor cortex, we noticed a heterogeneous layer-related distribution of mover. While relative mover abundance overall was below average, some cortical layers, i.e. layer I and layer V, showed higher mover intensity than other layers such as layer IV (Figure 1C, middle panel). At bregma $-3.5 \mathrm{~mm}$, mover was above average in all brain regions, i.e. periaqueductal grey, substantia nigra and ventral tegmental area $(47.31 \pm 1.99 \%, 32.11 \pm 2.71 \%$ and $8.81 \pm 1.74 \%$, respectively), while at the caudal-most level (bregma $-6.0 \mathrm{~mm}$ ), mover levels were again heterogeneous, being high in the molecular layer $(38.75 \pm 3.41 \%)$ and low in the granular layer of the cerebellum $(-34.68 \pm 1.13 \%)$. Overall, quantification revealed areas of increased and areas of decreased ratios of mover to synaptophysin compared to the average across the hemisphere. This corroborates the hypothesis that mover is differentially distributed throughout the adult mouse brain, and prompted us to investigate the pattern of mover in more detail within individual brain regions. 


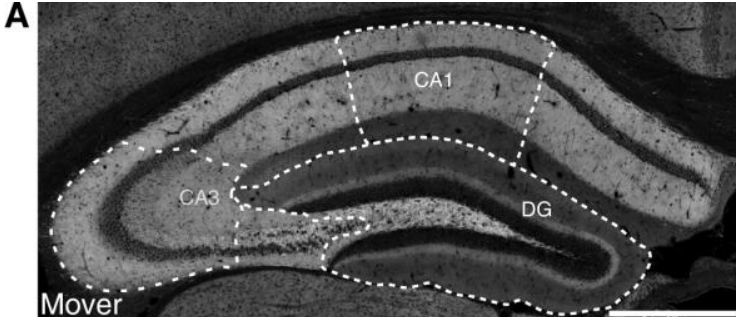

C

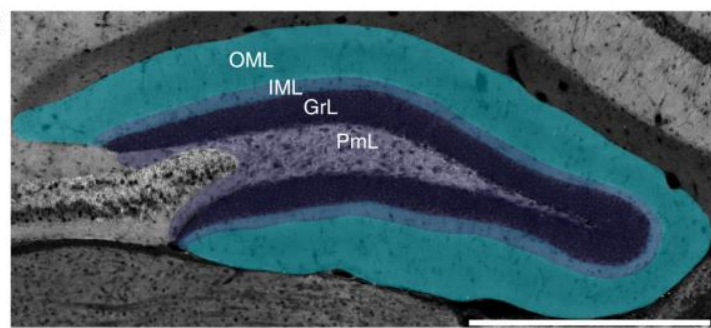

E

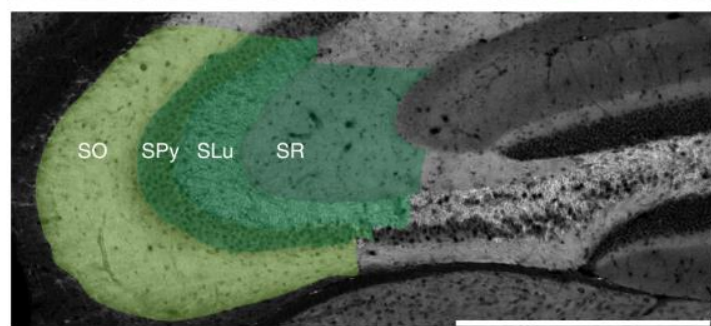

G

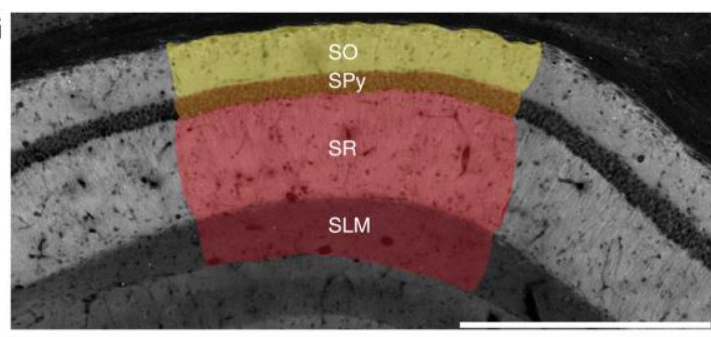

B

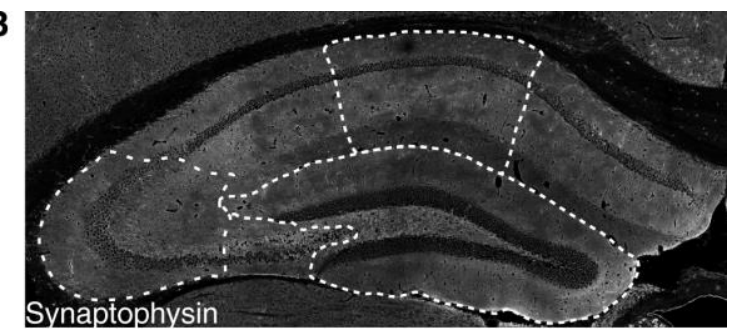

D

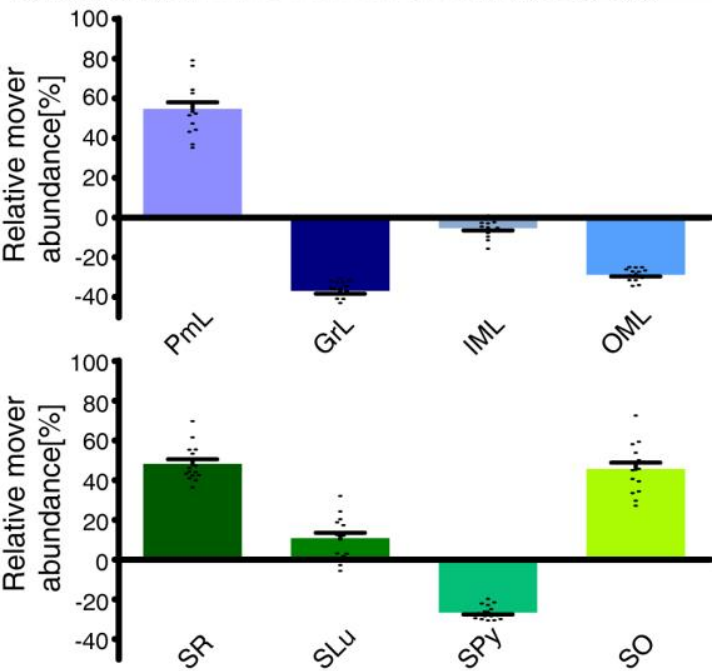

H

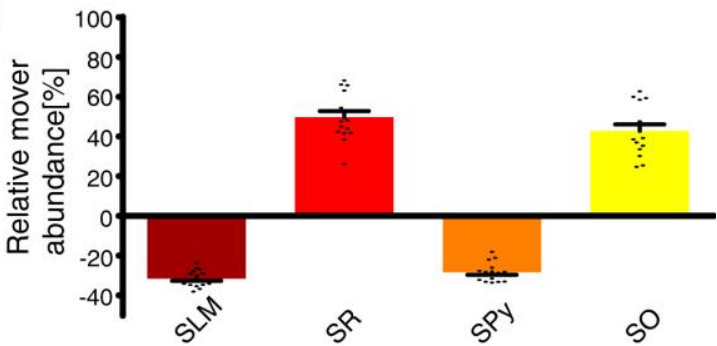

Figure 3: Mover distribution in the mouse hippocampus. Immunofluorescence staining of coronal slices of the mouse hippocampus. (A,B) Overview of the hippocampus showing the heterogeneous mover expression pattern (A) and the corresponding synaptophysin staining (B). The three regions of interest (DG, (C); CA3 (E); CA1 (G)) are delineated with white dotted lines. (D,F,H) Quantification comparing the ratio in the respective layer to the ratio of the whole hemisphere. Mover is especially abundant in the polymorph layer of DG (purple), stratum radiatum, lucidum and oriens of CA3 (dark green/green/neon green) and CA1(red/yellow). Levels of mover below average are detected in the granular, inner and outer molecular layer of DG (dark blue/grey/blue), stratum pyramidale of CA3 (light green) and CA1(orange) and stratum lacunosum-moleculare (dark red). OML, outer molecular layer; IML, inner molecular layer; GrL, granular layer; PmL, polymorph layer; SO, stratum oriens; Spy, stratum pyramidale; SLu, stratum lucidum; $\mathrm{SR}$, stratum radiatum; SLM, stratum lacunosum-moleculare. Scale bar $=500 \mu \mathrm{m}$. 


\section{Mover is differentially distributed in the different layers of the hippocampus}

When analyzing mover abundance in the different brain regions, we were surprised by the relatively low value obtained for the hippocampus: while mover staining seemed especially bright in that region, quantification yielded a value around 13\% above average. We also noted that mover was especially abundant in some layers of the hippocampus, while it seemed absent in others. We therefore determined mover-to-synaptophysin ratios in the different subregions and layers (Förster et al., 2006). While mover distribution was very heterogeneous throughout the hippocampus (Figure 3A), synaptophysin levels were fairly constant, with higher levels only in the big mossy fiber terminals in the polymorph layer of DG, also called the hilus, and stratum lucidum of CA3, and lower levels in the stratum pyramidale (Figure 3B). Analyzing the layers of the DG (Figure 3C), we saw high mover abundance in the polymorph layer ( $\mathrm{PmL} 54.72 \pm 2.90 \%$, purple), which includes mossy fibers. In contrast, mover abundance was below average in the granular and outer molecular layer (GrL $-36.93 \pm 1.27 \%$ and $\mathrm{OML}-28.86 \pm 0.71 \%$ respectively, dark blue/blue), while it was close to average in the inner molecular layer (IML $-5.35 \pm 1.02 \%$, grey; Figure 3D). In the CA3 region (Figure 3E), mover was highly abundant in the stratum radiatum and oriens (SR $48.19 \pm 2.07 \%$ and SO $45.68 \pm 2.79 \%$ respectively, dark green/neon green), while it was below average in the stratum pyramidale (SPy $-26.67 \pm 0.82 \%$, light green). In the stratum lucidum, where the mossy fibers from DG granule cells terminate onto the apical dendrites of CA3 pyramidal cells, mover abundance was $10.75 \pm 2.44 \%$ above average (SLu, green; Figure 3F). A detailed analysis of the CA1 region (Figure $\mathbf{3 G}$ ) yielded high mover ratios in the stratum radiatum, where Schaffer collaterals originating from CA3 pyramidal cells terminate onto pyramidal cells of CA1 (SR $49.70 \pm 2.73 \%$, red). In stratum oriens, mover abundance was also above average (SO $42.80 \pm 2.88 \%$, yellow), while both the stratum lacunosum-moleculare and pyramidale showed low levels of mover (SLM $-31.61 \pm 0.98 \%$ and SPy $-28.43 \pm 1.10 \%$ respectively, dark red/orange; Figure $\mathbf{3 H}$ ). Thus, mover is specifically associated with stratum radiatum, stratum oriens and the polymorph layer of DG, while it is close to average in the inner molecular layer of DG, and strikingly below average in the cell body layers and stratum lacunosummoleculare. 

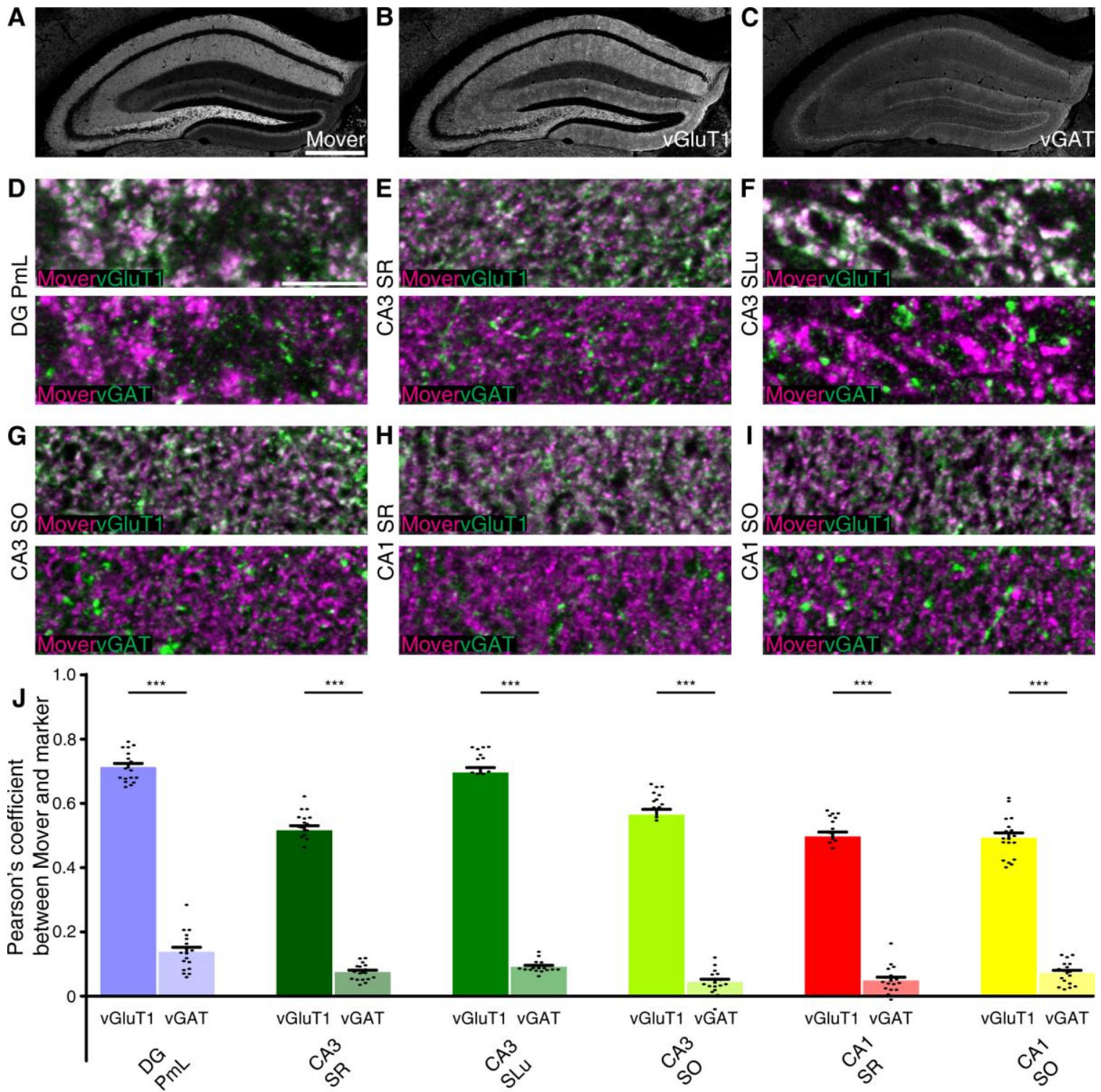

Figure 4: Mover colocalization with presynaptic markers vGlut1 and vGAT in the mouse hippocampus. Immunofluorescence triple labeling of the mouse hippocampus. (A-C) Overview showing the heterogeneous mover expression pattern (A) and the corresponding vGluT1 (B) and vGAT staining (C). (DI) Overlay of mover (magenta) and vGluT1 staining (green, upper panel) and mover (magenta) and vGAT (green, lower panel) in regions of the hippocampus where mover immunofluorescence was above average. (D) High colocalization of mover with vGluT1 in polymorph layer of DG, low colocalization of mover with vGAT. (E) High colocalization of mover with vGluT1 in stratum radiatum of CA3, low colocalization of mover with vGAT. (F) High colocalization of mover with vGluT1 in stratum lucidum of CA3, low colocalization of mover with vGAT. (G) High colocalization of mover with vGluT1 in stratum oriens of CA3, low colocalization of mover with vGAT. (H) High colocalization of mover with vGluT1 in stratum radiatum of CA1, low colocalization of mover with vGAT. (I) High colocalization of mover with vGluT1 in stratum oriens of CA1, low colocalization of mover with VGAT. (J) Visualization of the quantification of colocalization with the different markers using the Pearson's correlation coefficient. Bars show average \pm S.E.M; ${ }^{* * * P}<0.001$. PmL, polymorph layer of dentate gyrus; $S R$, stratum radiatum (of either CA3 or CA1); SLu, stratum lucidum of CA3; SO, stratum oriens (of either CA3 or CA1). Scale bar: (AC) $=500 \mu \mathrm{m},(\mathrm{D}-\mathrm{I})=10 \mu \mathrm{m}$ 


\section{Mover is present at excitatory synapses in the hippocampus}

To test whether this distributional heterogeneity also applies to synapse types, we triple-stained the hippocampus. We used the vesicular glutamate transporter 1 (vGluT1) as a marker for excitatory synapses (Ziegler et al., 2002). As a marker for inhibitory nerve terminals we used the vesicular y-aminobutyric acid (GABA) transporter (vGAT; Chaudhry et al., 1998). We focused on regions with high mover-to-synaptophysin ratios, and applied colocalization analysis using Pearson's correlation coefficient as a read-out. If two markers colocalize perfectly, Pearson's correlation coefficient would be 1 ; random distribution of signals or avoidance will yield low values, i.e. close to 0 or negative. As a means of quality check for our stainings, we performed the colocalization analysis between vGluT1 and VGAT, and detected low to very low Pearson's correlation coefficients (DG PmL: 0.169, Figure S1A; CA3 SR: 0.055, Figure S1B; CA3 SLu: 0.103, Figure S1C; CA3 SO: 0.027, Figure S1D; CA1 SR: 0.052, Figure S1E; CA1 SO: 0.074, Figure S1F; comparison of all values Figure S1G). The low Pearson's correlation values corroborate the quality of the staining and make colocalization analysis between mover and the different markers feasible (Figure 4). The rather high values in polymorph layer of DG (0.169) and stratum lucidum of CA3 (0.103) can be explained by the complex intermingling of GABAergic synapses and the extraordinarily large, excitatory mossy fiber terminals found in these regions. We therefore assume that in these regions, even these relatively high values reflect a lack of colocalization. Low magnification images of the individual channels of our triple stainings indicated similarities between the mover distribution (Figure 4A) and vGluT1 (Figure 4B). Accordingly, colocalization analysis of high magnification images yielded high values in all regions (DG PmL: 0.713, Figure 4D and S2A; CA3 SR: 0.516, Figure 4E and S2B; CA3 SLu: 0.696, Figure 4F and S2C; CA3 SO: 0.565, Figure 4G and S2D; CA1 SR: 0.497, Figure 4H and S2E; CA1 SO: 0.493, Figure $\mathbf{4 I}$ and S2F; comparison of all values Figure $\mathbf{4 J}$ ). The distribution patterns of mover and VGAT (Figure 4C) differed substantially from each other, which was also reflected in the low Pearson's correlation coefficient in all regions (DG PmL: 0.138, Figure 4D and S2A; CA3 SR: 0.075, Figure 4E and S2B; CA3 SLu: 0.091, Figure 4F and S2C; CA3 SO: 0.044, Figure 4G and S2D; CA1 SR: 0.049, Figure 4H and S2E; CA1 SO: 0.072, Figure 4 I and S2F; comparison of all values Figure 4J). 


\section{A}

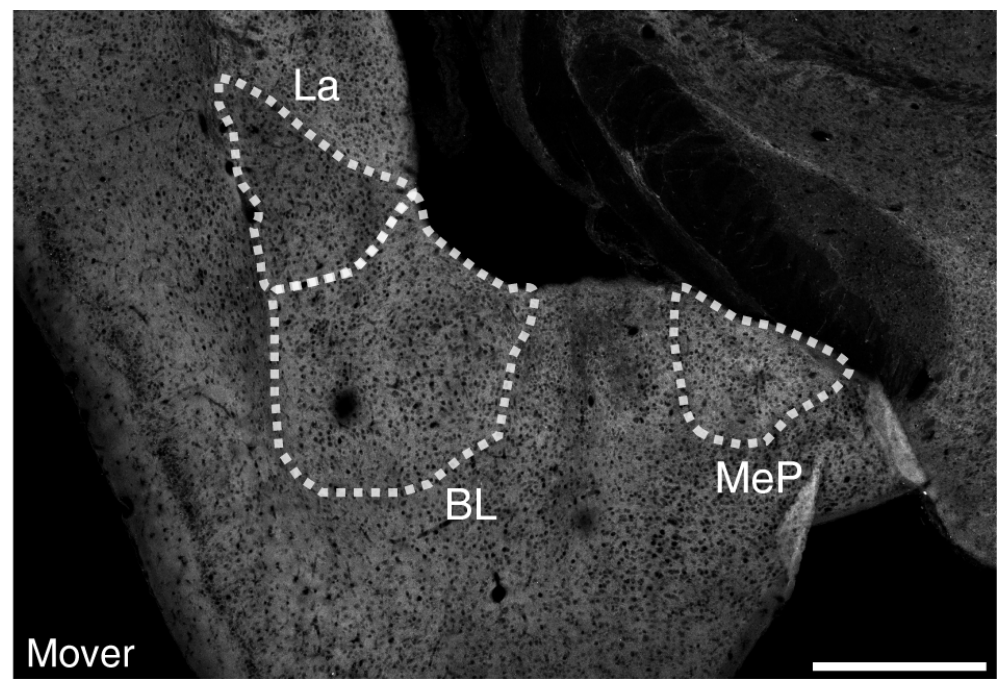

B
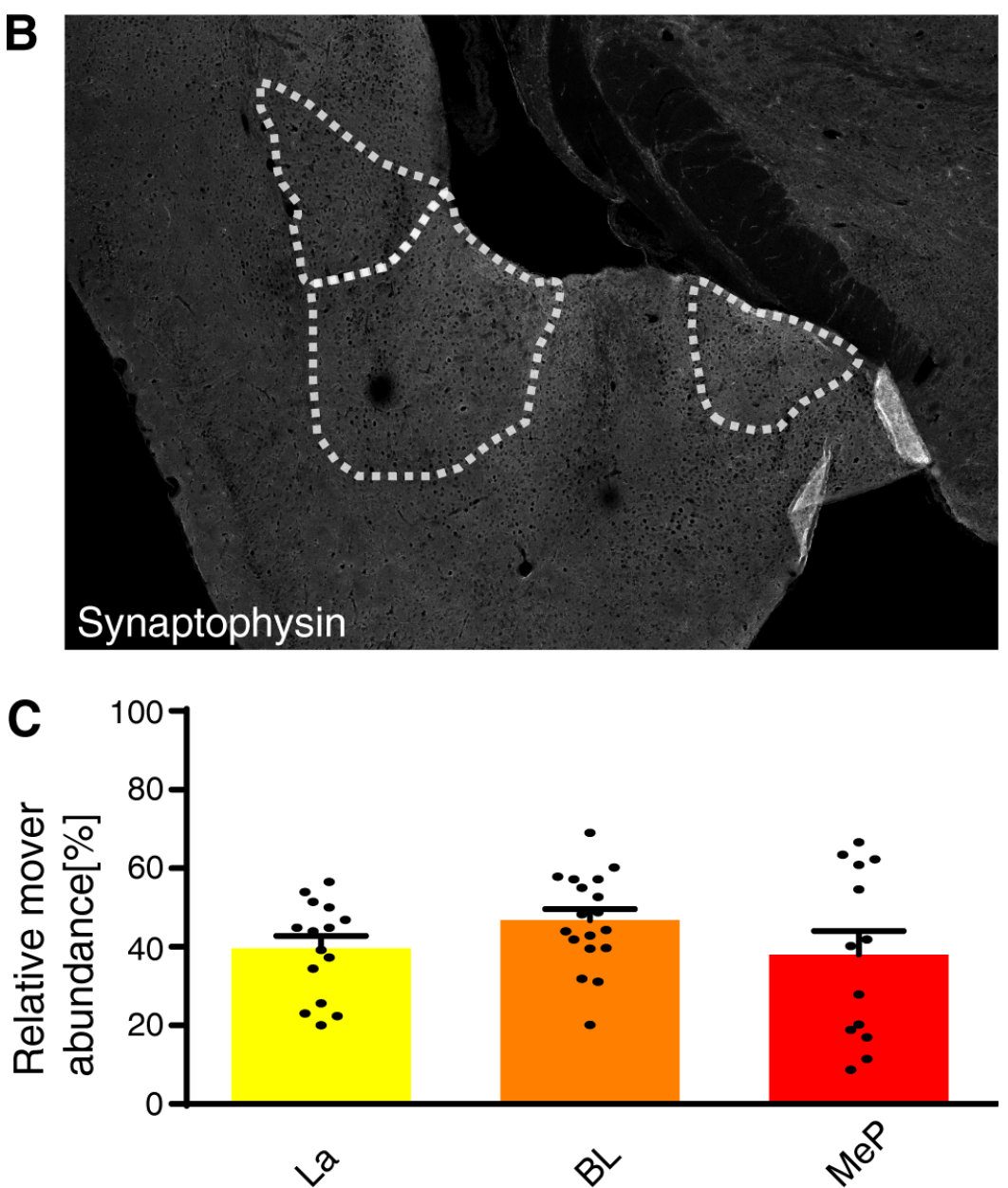

Figure 5: Mover distribution in the mouse amygdala. Immunofluorescence staining of coronal slices of the mouse amygdala. (A,B) Overview showing the heterogeneous mover expression pattern $(\mathbf{A})$ and the corresponding synaptophysin staining (B). The three regions of interest (La, BL, MeP) are delineated with white dotted lines. (C) Quantification comparing the ratio in the respective nuclei to the ratio of the whole hemisphere. High mover expression is detected in all three amygdaloid nuclei (La, yellow; BL, orange; MeP, red). Bars show average \pm S.E.M. La, lateral nuclei of the amygdala; BL, basolateral nuclei of the amygdala; MeP, medioposterior nuclei of the amygdala. Scale bar $=500 \mu \mathrm{m}$. 
The Pearson's correlation coefficient in DG PmL (0.138) did not exceed the value of vGluT1 and VGAT, and therefore likely indicated lack of colocalization. Colocalization between mover and vGluT1 was significantly higher than colocalization between mover and vGAT in all areas ( $p$ values: DG PmL: $4.3^{*} 10^{-27}$; CA3 SR: $1.37^{*} 10^{-25}$; CA3 SLu: $9.75^{*} 10^{-30}$; CA3 SO: $1.16^{*} 10^{-24}$; CA1 SR: $1.76 * 10^{-24}$; CA1 SO: $3.72 * 10^{-23}$ ). Taken together, these data suggest that in the hippocampus, mover is enriched at excitatory synapses, while it is absent from inhibitory synapses.

\section{Mover is enriched in the different amygdaloid nuclei}

Mover was even more abundant in the amygdala compared to the hippocampus (Figure 2E,F). Within the amygdala, mover and synaptophysin were homogeneously distributed among the different nuclei (Figure 5A,B). In the lateral amygdaloid nuclei (La), which receive glutamatergic input from sensory systems (Davis \& Whalen, 2001), mover abundance was high (39.61 $\pm 2.75 \%$, yellow, Figure $5 \mathrm{C}$ ). The same held true for the basolateral amygdala ( $\mathrm{BL} ; 46.73 \pm 2.75 \%$, orange), which receives input mainly from the auditory system, the hippocampus and the prefrontal cortex (Baars \& Gage, 2010; McDonald et al., 1996; McGarry \& Carter, 2017), and medioposterior amygdaloid nuclei (MeP; $37.98 \pm 4.92 \%$, red; Figure $5 \mathrm{C}$ ), which receives input from the main and accessory olfactory bulbs (Keshavarzi et al., 2014). In contrast to hippocampus, mover abundance was homogeneously high throughout the amygdala.

\section{Mover is present at both excitatory and inhibitory synapses in the amygdala}

As input into the amygdala is so diverse, we analyzed the colocalization of mover with vGluT1 and vGAT. Here again we checked for the Pearson's correlation coefficient between vGluT1 and VGAT as a means of quality control, and detected very low correlation values (La: 0.088, Figure S3A; BL: 0.073, Figure S3B; MeP: 0.074, Figure S3C; comparison of all values Figure S3D). Mover (Figure 6A) and vGluT1 (Figure 6B) colocalized in all three regions (La: 0.386, Figure 6D, and S4A; BL: 0.456 Figure 6E and S4B, MeP: 0.359; Figure 6F and S4C; comparison of all values Figure 6D), while colocalization with vGAT (Figure 6C) was strong in medioposterior nuclei $(0.419$, Figure $6 \mathrm{~F}$ and S4C), but weak in lateral (0.162, Figure 6D and S4A) and basolateral nuclei (0.140, Figure 6E and exceeded colocalization between mover and vGAT significantly ( $p$-values: La: $2.72 * 10^{-8}$; BL: $3.97^{*} 10^{-15}$ ), while in the medioposterior nucleus, colocalization between mover and VGAT was significantly higher than colocalization between mover and vGluT1 (p-value 0.043). Like in the hippocampus, mover is enriched at excitatory synapses throughout the amygdala. In contrast, synapses of the medioposterior nucleus display prominent mover S4B). 

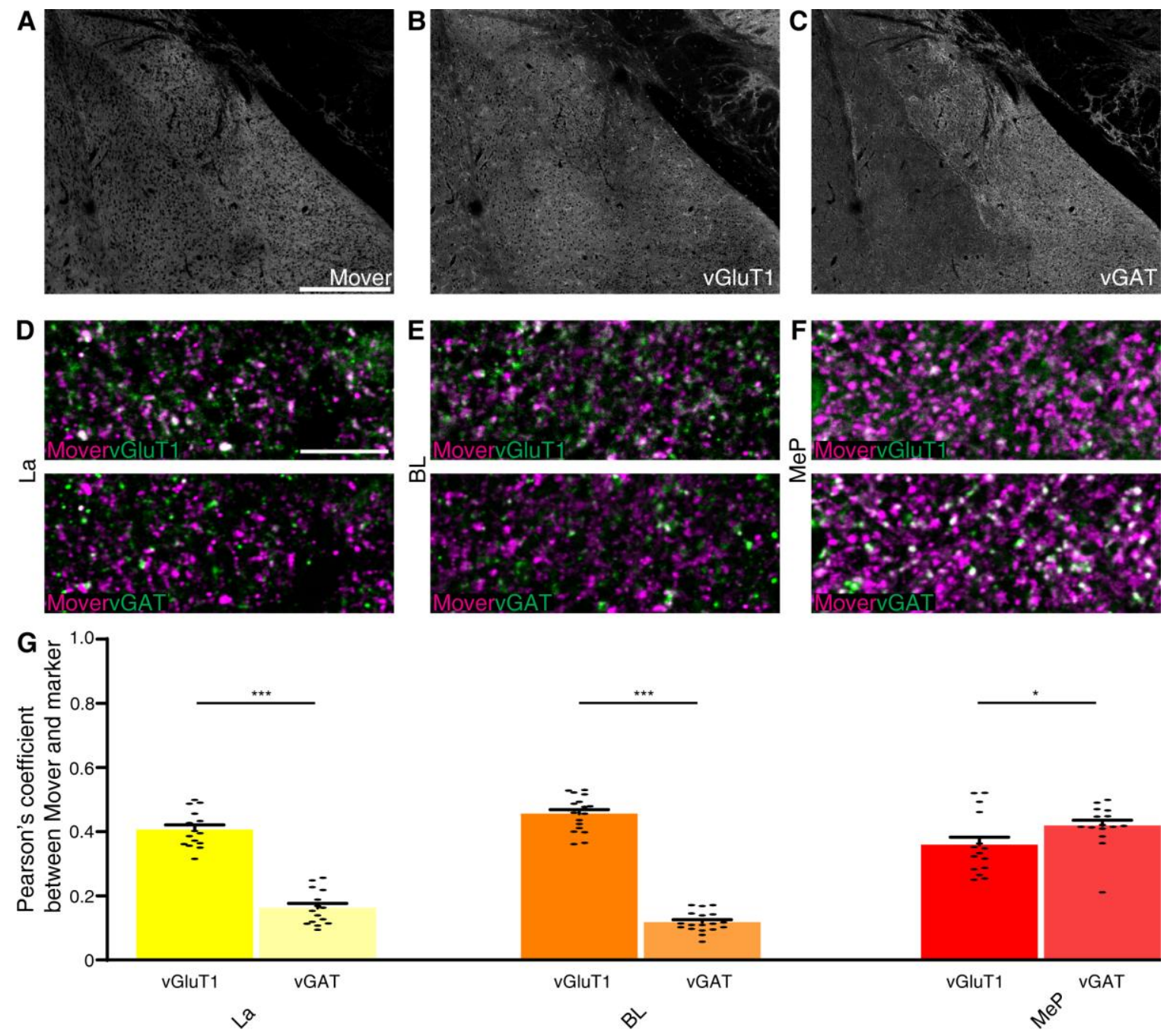

Figure 6: Mover colocalization with presynaptic markers vGlut1 and vGAT in the mouse amygdala. Immunofluorescence triple labeling of the mouse amygdala. (A-C) Overview showing the heterogeneous mover expression pattern (A) and the corresponding vGluT1 (B) and vGAT staining (C). (D-F) Overlay of mover (magenta) and vGluT1 staining (green, upper panel) and mover (magenta) and vGAT (green, lower panel) in the different nuclei of the amygdala. (D) Moderate colocalization of mover with vGlut1 in lateral amygdaloid nuclei, low colocalization of mover with vGAT. (E) Moderate colocalization of mover with vGlut1 in basolateral amygdaloid nuclei, low colocalization of mover with vGAT. (F) Moderate colocalization of mover with vGlut1 in medioposterior amygdaloid nuclei, moderate colocalization of mover with vGAT. (G) Visualization of the quantification of colocalization with the different markers using the Pearson's coefficient. Bars show average \pm S.E.M; ${ }^{*} P<0.05 ; * * *<0.001$. La, lateral nuclei of the amygdala; BL, basolateral nuclei of the amygdala; MeP, medioposterior nuclei of the amygdala. Scale bar: $(A-C)=500 \mu \mathrm{m},(D-F)=10 \mu \mathrm{m}$. 
In the lateral and basolateral nuclei, colocalization between mover and vGlutT1 staining. Overall, these data indicate that even though mover levels are high throughout the amygdala, mover is differentially distributed across synapse types in this brain region.

\section{Discussion}

With this study, we provide the first quantitative description of the distribution of mover, a vertebrate-specific synaptic vesicle protein we identified as a binding partner for the presynaptic scaffolding molecule bassoon (Ahmed et al., 2013; Thomas Kremer et al., 2007). A qualitative description had suggested that mover might be present at some synapses and absent from others. Here, we analyzed the protein levels and localization of mover in the adult mouse brain quantitatively. We found an unusually heterogeneous distribution, with high levels of mover in some regions, e.g. hippocampus and amygdala, and lower levels in other regions. Additionally, we analyzed the extent of colocalization of mover with vGluT1, a marker for excitatory synapses, and VGAT, a marker for inhibitory synapses. We discovered that also in respect to synapse types, mover shows a differential distribution. In particular, our study yielded three key observations:

First, mover is heterogeneously distributed among 16 brain regions. It is especially prominent in the ventral pallidum, septal nuclei and the amygdala. Second, mover is heterogeneously distributed within the hippocampus. Mover levels, compared to average in the hemisphere, are particularly high in the stratum radiatum and oriens, while mover is absent from the pyramidal cell layers and the stratum lacunosum-moleculare. On a synapse level, in all subregions of the hippocampus tested, mover is present at excitatory synapses, and absent from inhibitory synapses. Third, unlike its distribution in the hippocampus, mover levels are homogeneously high throughout the amygdala. On a synapse level, mover is present at excitatory synapses in all subregions of the amygdala, but differentially distributed among inhibitory synapses: a high Pearson's value for mover and VGAT (0.419) indicates the presence of mover at inhibitory synapses in the medioposterior nucleus, while low Pearson's values indicate that mover is absent from most inhibitory synapses in the lateral and basolateral amygdala (0.162 and 0.140 respectively). Overall, our study reveals that mover is indeed differentially distributed among synapses in the adult mouse brain, and that its association with inhibitory synapses differs between brain regions and even within one brain region, i.e. the amygdala. These data raise the possibility that mover may act as a region-specific and synapse-specific regulator of synaptic transmission.

To determine the relative levels of mover protein we immunostained brain sections with a polyclonal mover antiserum used previously for western blotting, co-immunoprecipitation and 
immunogold labelling of synaptic vesicles (Ahmed et al., 2013). Determining absolute protein amounts using this antibody is impossible with indirect immunofluorescence techniques. Confocal microscopy on the other hand allowed us to readily obtain a quantitative readout of the relative levels of mover in a large number of brain regions and its association with excitatory and inhibitory synapses. Therefore, this is the ideal technique for testing the hypothesis that mover is differentially distributed among brain regions and synapse types.

By using synaptophysin as a reference marker we accounted for two principle caveats of our approach: first, the overall intensity of immunofluorescence varies between experiments. This is a general feature of immunofluorescence. Second, increased mover immunofluorescence in a certain brain region compared to its surroundings may represent increased levels of mover, or simply reflect an increased density of synapses or increased number of synaptic vesicles per terminal in that region. We chose synaptophysin as a reference marker, as it is present at every synapse and likely on all synaptic vesicles. Thus, synaptophysin levels within a single synapse are directly proportional to the size (i.e. the synaptic vesicles content) of this synapse (Navone et al., 1986). Because of these features, synaptophysin is widely used as a general synapse marker (Barak et al., 2010; Micheva et al., 2010). We cannot exclude the possibility that, unexpectedly, the abundance of synaptophysin itself is particularly high or low in certain brain areas. However, our immunostainings do not support this possibility, as the synaptophysin staining intensity was indeed rather uniform across the entire hemispheres, as expected. We conclude that by determining the ratio between mover and synaptophysin we can correct for differences in synapse density and synapse size in a certain brain area. In addition, comparing the ratio obtained for an individual brain region to the ratio in the whole hemisphere quantifies the abundance of mover relative to average in the hemisphere and corrects for differences in overall staining intensity between experiments. Note that we do not compare brain areas located at distinct levels along the rostro-caudal axis, because the average amount of mover differs at the different levels and depends on the number of regions with high mover intensity. For example, at a level with few high intensity regions, the lowest mover to synaptophysin ratio might be $25 \%$ compared to average, representing the complete absence of mover, while absence is represented by a value of $-40 \%$ at a level with more high intensity regions. Comparing different levels is thus not meaningful. We therefore exclusively compare brain areas and their subregions at a certain level to the average of the hemisphere at the same level. Using this stringent approach, we found that at the first level that we analyzed (the most rostral level; see Figure 1) mover was particularly abundant in islands of Calleja; at the second level, it was most abundant in the septal nuclei and ventral pallidum; at the third level, it was highest in the amygdala; at the 
two most caudal levels, it was highest in the periaqueductal grey and the molecular layer of the cerebellum. All levels except the level including the periaqueductal grey, substantia nigra and the ventral tegmental area also contained regions where mover was below average, further emphasizing its heterogeneous distribution.

To quantify colocalization we determined the Pearson's correlation coefficient (further methodological discussion see Suppl. Info). Pearson's coefficients for VGluT1 and vGAT were between 0.027 and 0.169 , depending on the brain area and subregions. To apply a maximally stringent criterion, we used the Pearson's value for vGluT1 and VGAT from each individual subregions as a threshold, and we considered mover as "not colocalized" with a marker when the Pearson's value was the same or below the value for vGluT1 and vGAT in the same region. Using this criterion, mover is clearly present at excitatory synapses throughout the hippocampus and amygdala (Pearson's values were between 0.359 and 0.713 for the subregions). In addition, mover is present at inhibitory synapses in the amygdala, with the highest colocalization with vGAT in the medioposterior amygdala (Pearson's value of 0.419 ). In contrast, mover is clearly absent from inhibitory synapses in most subregions of the hippocampus, and likely absent in the stratum radiatum and oriens of the CA3, too. In these two CA3 layers, the Pearson's coefficient for mover and vGAT was strikingly low 0.075 in the stratum radiatum and 0.044 in the stratum oriens). But since the Pearson's coefficient for vGlut1 and vGAT was even lower in these regions ( 0.055 in the stratum radiatum and 0.027 in the stratum oriens) we cannot exclude that there is some degree of colocalization for mover with vGAT in these two areas. Note, however, that these Pearson's values are very low, indicating that even in these regions only a small fraction of inhibitory synapses, if any, have mover. Overall, these data suggest that mover primarily regulates excitatory synaptic transmission in the hippocampus.

In the hippocampus, the abundance of mover varies strikingly among the layers, suggesting that mover may be particularly important for certain hippocampal synapses. Throughout the hippocampus, the levels of mover compared to synaptophysin are very low in the cell body layers, i.e. in the stratum granulosum of the dentate gyrus and the stratum pyramidale of the CA3 and CA1. This is consistent with its absence from inhibitory terminals, which are arranged as perisomatic synapses in the cell body layers. Interestingly, mover levels are equally low in the outer molecular layer of the dentate gyrus and the stratum lacunosum-moleculare of the CA1. These are layers of the hippocampus that receive input from the entorhinal cortex, i.e. from outside the hippocampus. In contrast, mover levels compared to synaptophysin are most strikingly above average in the polymorph layer of the dentate gyrus, also called the hilus, as well as the stratum radiatum and the stratum oriens of the CA3 and CA1. These are three layers 
that contain axons and axon terminals arising from the principle cells of the hippocampus: the polymorph layer contains the glutamatergic axons of dentate gyrus granule cells, called mossy fibers. Collaterals of these mossy fibers make synapses within the polymorph layer, by targeting excitatory mossy cells and inhibitory basket cells. The mossy cell axons synapse in the inner molecular layer of the dentate gyrus, the basket cells synapse on the granule cell somata. Thus, through mossy fibers collaterals, dentate gyrus granule cells trigger both excitatory and inhibitory feedback onto themselves. The high levels of mover in the polymorph region suggest a role for mover in mossy fiber axon collaterals, i.e. in presynaptic terminals targeting basket cells or mossy cells. In each case, mover would be important for the regulation of feedback loops within the dentate gyrus. The stratum radiatum and oriens contain the glutamatergic axons of the hippocampal pyramidal cells: these axons are called associational/commissural fibers when they target pyramidal cell dendrites within either CA3 or CA1, and they are called Schaffer collaterals when they run from the CA3 to the CA1 region. The fact that mover levels compared to synaptophysin are high in these regions, while they are low in the outer molecular layer of the dentate gyrus and the stratum lacunosum-moleculare, suggest that mover may be more important for the regulation of intra-hippocampal information flow and processing than for the entry of signals into the dentate gyrus and hippocampus.

Mover is present in mossy fiber terminals in the stratum lucidum of the CA3 (Thomas Kremer et al., 2007), and its abundance is slightly above average in these synapses, as indicated by our current study. Mossy fiber terminals are specialized nerve endings with a low release probability and strong capacity for facilitation (Nicoll \& Schmitz, 2005). Their plasticity is regulated by an evolutionarily conserved presynaptic protein called tomosyn: knockdown of tomosyn in mossy fibers reduces presynaptic short-term and long-term potentiation, presumably by increasing basal release probability (Ben-Simon et al., 2015). Tomosyn is present in mossy fiber terminals, but absent from inhibitory terminals (Barak et al., 2010). The absence of mover from inhibitory terminals and presence at mossy fiber terminals is reminiscent of the distribution of tomosyn. In addition, mover regulated release probability and short-term plasticity at the calyx of Held, a specialized axo-somatic synapse located in the brainstem: knockdown of mover at the calyx of Held increases short-term depression and release probability (Körber et al., 2015). If mover had a similar role at mossy fiber terminals, it could add a vertebrate-specific function in regulating presynaptic plasticity to the established role of the conserved protein tomosyn. Knockout studies should throw light on the function of mover at these specialized synapses, and on its potential role for spatial learning and memory. 
A similar layer-specific distribution of mover was detectable in neocortical fields, such as the primary somatosensory cortex. The primary somatosensory cortex, also called barrel cortex, is a highly organized structure that mediates touch and pain sensation from the whisker pad. The barrels are organized in columns and rows (Schubert et al., 2007). Whiskers are represented somatotopically, meaning that one barrel in a row represents the corresponding whisker in that row in the whisker pad (Welker et al., 1974). Input into the barrel cortex originates from two distinct thalamic nuclei, the ventral posteromedial nucleus (VPm) and the posteromedial nucleus (POm). Fibers from the VPm form the lemniscal pathway and mainly project to the barrels in layer IV, while fibers from POm, which form the paralemniscal pathway, target layer Va pyramidal cells and layer I neurons to a smaller extent (Bosman et al., 2011). While the touchmediating function of the lemniscal pathway has been described in detail (e.g. Nicolelis, 2005; Yu et al., 2006), the whole extent of functions of the paralemniscal pathway remains unclear, ranging from modulation of the lemniscal pathway (Ahissar et al., 2000) to pain sensation (Frangeul et al., 2014). Our stainings revealed higher mover intensities in layer $V$ of the primary somatosensory cortex and lower mover intensity in layer IV. This suggests a pathway-specific expression of mover in the paralemniscal pathway, which can potentially be used as a marker specific for this pathway, something that has been missing so far. In future experiments it remains to be seen whether mover also exhibits a pathway-specific function, such as modulating pain sensation or fine-tuning of touch sensation. Functional experiments, such as electrophysiological measurements and (in vivo) calcium imaging should shed light on this question and determine mover's function in this pathway.

Unlike its heterogeneous distribution within the hippocampus and the neocortex, mover levels were homogeneously high, i.e. above average of the hemisphere, in the amygdala. Input into the different nuclei is diverse, just like the function of the amygdala. It has been connected to fear conditioning (LeDoux et al., 1990) hormone secretion (Eleftheriou \& Zolovick, 1967) and emotional and sexual behavior (Kondo, 1992). In humans, the amygdala has even been described to be involved in psychiatric disorders, such as posttraumatic stress disorder (PTSD; Mahan \& Ressler, 2012). The amygdala has been described as a very plastic structure (Sangha \& Maren, 2015). The medial amygdaloid nucleus receives input from the main and accessory olfactory bulbs, which is involved in mediating socio-sexual behavior (Fernandez-Fewell \& Meredith, 1994). Within this nucleus, glutamatergic and GABAergic neurons can be found. Both cell types project to the hypothalamus, but there is a subpopulation of GABAergic neurons which function as local circuit interneurons and most likely provide feedforward inhibition onto the excitatory neurons in the medioposterior amygdala. The high abundance of mover and high 
Pearson's values for colocalization with vGAT suggest that mover might be involved in this local circuitry, shaping the input from the olfactory bulb to the medial amygdala and its output to the hypothalamus.

Most strikingly, mover is associated with inhibitory synapses in the amygdala, while it is absent from inhibitory synapses in the hippocampus. This raises the possibility that mover may act as a regulator of synapse function and plasticity with particular importance for the heterogeneity of inhibitory synapses, and may thus contribute to a proper excitation-inhibition balance.

Except for its association with the hippocampus and amygdala, mover was especially abundant in the ventral pallidum and septal nuclei of the adult mouse brain. Both areas have been connected to reward and reinforcement, and the ventral pallidum has also been suggested to be involved in addiction. High levels of mover in these regions could suggest a role for mover in these processes, and warrant further investigation of mover's role in reward and addiction.

We found mover as an interaction partner for the active zone scaffolding protein bassoon in a yeast-2-hybrid assay (Thomas Kremer et al., 2007) and later showed that it is associated with SVs (Ahmed et al., 2013). Interestingly, probing purified SVs with immunogold electron microscopy, mover was associated with only 16 percent of the SVs, while synaptophysin was associated with virtually all SVs (Ahmed et al., 2013). The strong association of synaptophysin with SVs lends further support to our assumption that synaptophysin is a faithful marker for SVs. The association of mover with only a fraction of purified SVs may indicate that mover is selectively attached to a subset of SVs in a given synapse, but it is also consistent with our observation that some synapses have very low levels of mover, or even no mover.

Mover and bassoon are two of a remarkably small number of proteins that are not evolutionarily conserved but rather evolved in a manner unique to vertebrates. Among these proteins are the active zone scaffolding piccolo, the motor adaptor syntabulin and the synaptic vesicle protein synuclein (Gundelfinger et al., 2016; Cai et al., 2007; George, 2002). Whether these proteins confer certain vertebrate-specific functions to the conserved core machinery of neurotransmitter release is an open question. Double knockdown of bassoon and piccolo leads to disassembly of synapses, suggesting that these two multi-domain scaffolding proteins stabilize vertebrate synapses (Waites et al., 2013). Other vertebrate-specific proteins are thought to increase the functional heterogeneity of synapses in the brain (Emes et al., 2008; Ryan \& Grant, 2009). A heterogeneous expression, such as that revealed for mover here, is expected as a key feature of such modulatory proteins. At the Calyx of Held synapse, short term depression and release probability are increased after knockdown of mover, suggesting that at 
least one of the roles of mover is to regulate release probability and short-term plasticity (Körber et al., 2015). Release probability was also increased at the endbulb of Held synapse in mice expressing mutant bassoon (Mendoza Schulz et al., 2014). In contrast, release probability was unaffected in bassoon knockout mice at the cerebellar mossy fiber synapse, while SV reloading was impaired (Stefan Hallermann et al., 2010). Our observation that mover is heterogeneously expressed, in combination with the interaction of mover with bassoon (Thomas Kremer et al., 2007) and its association with a subset of SVs (Ahmed et al., 2013), raises the possibility that the levels of mover may regulate the interaction of bassoon with SVs at active zones, thus contributing to presynaptic heterogeneity. Functional studies involving knock-out and knock-in models of mover, employing electrophysiological and biochemical methods, are required to analyze the presynaptic pathways modulated by mover.

While high mover abundance could indicate crucial mover functions, regions with low expression levels of mover should not be disregarded. Mover abundance in the anterior cingulate cortex, for example, was below average in the adult mouse brain. However, in human schizophrenic patients, mover has been shown to be upregulated in the anterior cingulate cortex (Clark et al., 2006), raising the possibility that mover may be regulated by neuronal activity. For example, aberrant neuronal activity associated with schizophrenia could upregulate mover. If mover dampens presynaptic release, as suggested by knockdown at the calyx of Held (Körber et al., 2015), its activity-dependent upregulation could occur as a protective mechanism to confine runaway excitation.

Further studies should reveal whether activity-dependent expression contributes to the remarkably heterogeneous distribution of mover. In any case, its differential association with synapses on the level of brain areas, subregions and types of synapses renders it a candidate for a protein that generates synaptic heterogeneity.

\section{Conflict of Interest}

The authors declare that the research was conducted in the absence of any commercial or financial relationships that could be construed as a potential conflict of interest.

\section{Author Contributions}

RW performed experiments, analyzed data, contributed to experimental design and prepared the manuscript. TD designed the studies and the entire project and also prepared the manuscript. Both authors discussed the results, read and approved the final manuscript. 


\section{Funding}

This study was funded by the DFG Research Center for Nanoscale Microscopy and Molecular Physiology of the Brain (CNMPB, B1-1, to T.D.).

\section{Acknowledgements}

We thank Irmgard Weiß for expert technical assistance. We also thank the European Neuroscience Institute (ENI-G, Goettingen, Germany) and especially Dr. Nils Halbsgut for providing the LSM800 and support and technical assistance with the setup. Additionally, we thank Dr. Julio Viotti for helpful advice and fruitful discussions.

\section{References}

Adler, J., \& Parmryd, I. (2010). Quantifying colocalization by correlation: The pearson correlation coefficient is superior to the Mander's overlap coefficient. Cytometry Part A, 77(8), 733742.

Ahissar, E., Sosnik, R., \& Haldarilu, S. (2000). Transformation from temporal to rate coding in a somatosensory thalamocortical pathway. Nature, 406(6793), 302-306.

Ahmed, S., Wittenmayer, N., Kremer, T., Hoeber, J., Kiran Akula, A., Urlaub, H., ... Dresbach, T. (2013). Mover Is a Homomeric Phospho-Protein Present on Synaptic Vesicles. PLoS $O N E, 8(5)$.

Antonini, D., Dentice, M., Mahtani, P., De Rosa, L., Gatta, G. Della, Mandinova, A., ... Missero, C. (2008). Tprg, a gene predominantly expressed in skin, is a direct target of the transcription factor p63. Journal of Investigative Dermatology, 128(7), 1676-1685.

Baars, B. J., \& Gage, N. M. (2010). Cognition, Brain and Consciousness(2nd ed.). Burlington, MA 01803, USA: Elsevier Inc.

Barak, B., Williams, A., Bielopolski, N., Gottfried, I., Okun, E., Brown, M. A., ... Ashery, U. (2010). Tomosyn Expression Pattern in the Mouse Hippocampus Suggests Both Presynaptic and Postsynaptic Functions. Frontiers in Neuroanatomy, 4(12), 1-11.

Ben-Simon, Y., Rodenas-Ruano, A., Alviña, K., Lam, A. D., Stuenkel, E. L., Castillo, P. E., \& Ashery, U. (2015). A Combined Optogenetic-Knockdown Strategy Reveals a Major Role of Tomosyn in Mossy Fiber Synaptic Plasticity. Cell Reports, 12(3), 396-404.

Blackman, A. V., Abrahamsson, T., Costa, R. P., Lalanne, T., \& Sjöström, P. J. (2013). Target-cellspecific short-term plasticity in local circuits. Frontiers in Synaptic Neuroscience, 5(12), 113. 
Bosman, L. W. J., Houweling, A. R., Owens, C. B., Tanke, N., Shevchouk, O. T., Rahmati, N., ... De Zeeuw, C. I. (2011). Anatomical Pathways Involved in Generating and Sensing Rhythmic Whisker Movements. Frontiers in Integrative Neuroscience, 5(12), 1-28.

Burré, J., Beckhaus, T., Corvey, C., Karas, M., Zimmermann, H., \& Volknandt, W. (2006). Synaptic vesicle proteins under conditions of rest and activation: Analysis by 2-D difference gel electrophoresis. Electrophoresis,27(17), 3488-3496.

Cai, Q., Pan, P.-Y., \& Sheng, Z.-H. (2007). Syntabulin-Kinesin-1 Family Member 5B-Mediated Axonal Transport Contributes to Activity-Dependent Presynaptic Assembly. Journal of Neuroscience, 27(27), 7284-7296.

Chaudhry, F. a., Reimer, R. J., Bellocchio, E. E., Danbolt, N. C., Osen, K. K., Edwards, R. H., \& Storm-Mathisen, J. (1998). The vesicular GABA transporter, VGAT, localizes to synaptic vesicles in sets of glycinergic as well as GABAergic neurons. Journal of Neuroscience, 18(23), 9733-9750.

Clark, D., Dedova, I., Cordwell, S., \& Matsumoto, I. (2006). A proteome analysis of the anterior cingulate cortex gray matter in schizophrenia. Molecular Psychiatry, 11, 459-470.

Davis, M., \& Whalen, P. J. (2001). The amygdala: Vigilance and emotion. Molecular Psychiatry, $6(1), 13-34$.

Dunn, K. W., Kamocka, M. M., \& McDonald, J. H. (2011). A practical guide to evaluating colocalization in biological microscopy. Am J Physiol Cell Physiol, 300, C723-C742.

Eleftheriou, B. E., \& Zolovick, A. J. (1967). Effect of amygdaloid lesions on plasma and pituitary levels of luteinizing hormone. J. Reprod. Fert., 14, 33-37.

Emes, R. D., Pocklington, A. J., Anderson, C. N. G., \& Bayes, A. (2008). Evolutionary expansion and anatomical specialization of synapse proteome complexity. Nat Neuroscience, 11(7), 799-806.

Fejtova, A., \& Gundelfinger, E. (2006). Molecular Organization and Assembly of the Presynaptic Active Zone of Neurotransmitter Release. In Cell Communications in Nervous and Immune System. Results and Problems in Cell Differentiation. Springer, Berlin, Heidelberg.

Fernandez-Fewell, G. D., \& Meredith, M. (1994). C-Fos Expression in Vomeronasal Pathways of Mated or Pheromone-Stimulated Male Golden Hamsters: Contributions From Vomeronasal Sensory Input and Expression Related To Mating Performance. Journal of Neuroscience, 14(6), 3643-3654.

Förster, E., Zhao, S., \& Frotscher, M. (2006). Laminating the hippocampus. Nature Reviews Neuroscience, 7(4), 259-267. 
Frangeul, L., Porrero, C., Garcia-Amado, M., Maimone, B., Maniglier, M., Clascá, F., \& Jabaudon, D. (2014). Specific activation of the paralemniscal pathway during nociception. European Journal of Neuroscience, 39(9), 1455-1464.

George, J. M. (2002). The synucleins. Genome Biology, 3(1), 1-6.

Gundelfinger, E. D., Reissner, C., \& Garner, C. C. (2016). Role of Bassoon and Piccolo in Assembly and Molecular Organization of the Active Zone. Frontiers in Synaptic Neuroscience, 7(1).

Hallermann, S., Fejtova, A., Schmidt, H., Weyhersmüller, A., Silver, R. A., Gundelfinger, E. D., \& Eilers, J. (2010). Bassoon speeds vesicle reloading at a central excitatory synapse. Neuron, 68(4), 710-723.

Keshavarzi, S., Sullivan, R. K. P., lanno, D. J., \& Sah, P. (2014). Functional Properties and Projections of Neurons in the Medial Amygdala. Journal of Neuroscience, 34(26), 86998715.

Kondo, Y. (1992). Lesions of the medial amygdala produce severe impairment of copulatory behavior in sexually inexperienced male rats. Physiology\&Behavior, 51(5), 939-943.

Körber, C., Horstmann, H., Venkataramani, V., Herrmannsdörfer, F., Kremer, T., Kaiser, M., ... Kuner, T. (2015). Modulation of Presynaptic Release Probability by the Vertebrate-Specific Protein Mover. Neuron, 87(3), 521-533.

Kremer, T., Kempf, C., Wittenmayer, N., Nawrotzki, R., Kuner, T., Kirsch, J., \& Dresbach, T. (2007). Mover is a novel vertebrate-specific presynaptic protein with differential distribution at subsets of CNS synapses. FEBS Letters, 581(24), 4727-4733.

LeDoux, J. E., Cicchetti, P., Xagoraris, A., \& Romanski, L. M. (1990). The Lateral Amygdaloid in Fear Conditioning Nucleus : Sensory Interface Amygdala. Journal of Neuroscience, 10(4), 1062-1069.

Mahan, A. L., \& Ressler, K. J. (2012). Fear conditioning, synaptic plasticity, and the amygdala: implications for posttraumatic stress disorder. Trends Neurosci., 35(1), 24-35.

McDonald, A. J., Mascagni, F., \& Guo, L. (1996). Projections of the medial and lateral prefrontal cortices to the amygdala: A Phaseolus vulgaris leucoagglutinin study in the rat. Neuroscience, 71(1), 55-75.

McGarry, L. M., \& Carter, A. G. (2017). Prefrontal Cortex Drives Distinct Projection Neurons in the Basolateral Amygdala. Cell Reports,21(6), 1426-1433.

Mendoza Schulz, A., Jing, Z., María Sánchez Caro, J., Wetzel, F., Dresbach, T., Strenzke, N., ... Moser, T. (2014). Bassoon-disruption slows vesicle replenishment and induces homeostatic plasticity at a CNS synapse. EMBO Journal, 33(5), 512-527. 
Micheva, K. D., Busse, B., Weiler, N. C., O’Rourke, N., \& Smith, S. J. (2010). Single-synapse analysis of a diverse synapse population: Proteomic imaging methods and markers. Neuron, 68(4), 639-653.

Navone, F., Jahn, R., Gioia, G. Di, Stukenbrok, H., Greengard, P., \& Camilli, P. De. (1986). Protein p38: An integral membrane protein specific for small vesicles of neurons and neuroendocrine cells. Journal of Cell Biology, 103(6), 2511-2527.

Nicolelis, M. A. L. (2005). Computing with thalamocortical ensembles during different behavioural states. Journal of Physiology, 566(1), 37-47.

Nicoll, R. A., \& Schmitz, D. (2005). Synaptic plasticity at hippocampal mossy fibre synapses. Nature Reviews Neuroscience, 6(11), 863-876.

Okerlund, N. D., Schneider, K., Leal-Ortiz, S., Montenegro-Venegas, C., Kim, S. A., Garner, L. C., ... Garner, C. C. (2017). Bassoon Controls Presynaptic Autophagy through Atg5. Neuron, 93(4), 897-913.e7.

Paxinos, G., \& Franklin, K. B. J. (2001). The Mouse Brain in Stereotaxic Coordinates(2nd editio). San Diego: ACADEMIC PRESS.

Ryan, T. J., \& Grant, S. G. N. (2009). The origin and evolution of synapses. Nature Reviews Neuroscience, 10(11), 701-712.

Sangha, S., \& Maren, S. (2015). Plasticity of Fear and Safety Neurons of the Amygdala in Response to Fear Extinction. Frontiers in Behavioral Neuroscience, 9(12), 1-10.

Schubert, D., Kötter, R., \& Staiger, J. F. (2007). Mapping functional connectivity in barrel-related columns reveals layer- and cell type-specific microcircuits. Brain Structure and Function, 212(2), 107-119.

Südhof, T. C. (2012). The presynaptic active zone. Neuron,75(1), 11-25.

Waites, C. L., Leal-Ortiz, S. A., Okerlund, N., Dalke, H., Fejtova, A., Altrock, W. D., ... Garner, C. C. (2013). Bassoon and Piccolo maintain synapse integrity by regulating protein ubiquitination and degradation. EMBO Journal, 32(7), 954-969.

Welker, C. (1971). Microelectrode delineation of fine grain somatotopic organization of (Sml) cerebral neocortex in albino rat. Brain Res., 26(2), 259-75.

Welker, C., \& Woolsey, T. A. (1974). Structure of layer IV in the somatosensory neocortex of the rat: Description and comparison with the mouse. Journal of Comparative Neurology, 158(4), 437-453.

Yu, C., Derdikman, D., Haidarliu, S., \& Ahissar, E. (2006). Parallel thalamic pathways for whisking and touch signals in the rat. PLoS Biology, 4(5), 819-825. 
Ziegler, D. R., Cullinan, W. E., \& Herman, J. P. (2002). Distribution of vesicular glutamate transporter mRNA in rat hypothalamus. Journal of Comparative Neurology, 448(3), 217229.

(This page intentionally left blank) 


\title{
4 MOVER IS DIFFERENTIALLY
}

\section{EXPRESSED AT ENDBULBS OF HELD}

\author{
AND INHIBITORY SYNAPSES
}

\section{TARGETING BUSHY CELLS IN THE}

\section{VCN}

Manuscript

\section{ABSTRACT}

"Bushy cells (BCs) in the ventral cochlear nucleus (VCN) receive excitatory endbulbs of Held from auditory nerve fibers (ANFs) and inhibitory synapses from the dorsal cochlear nucleus. This network forms the first relay station of the binaural that mediates sound encoding and ultimately enables us to hear. We investigated the presence and structural effect of the vertebrate-specific synaptic vesicle (SV) protein Mover that has been implied in the modulation of synaptic release probability and plasticity in the calyx of Held and the hippocampal mossy fibers. We find that Mover is differentially expressed across excitatory and inhibitory synapses targeting BCs and that a knockout of Mover induces opposite effects in these two synapse types. Thereby, Mover has a diametrically opposed function in inhibitory synapses compared to excitatory synapses." (Wallrafen et al., in preparation) 


\section{Authors}

Rebecca Wallrafen, Thomas Dresbach

Synaptogenesis group, Institute of Anatomy and Embryology, University Medical Center Göttingen, Kreuzbergring 36, 37075 Göttingen, Germany

Running title: Mover in the mouse VCN

Keywords: Auditory brainstem/Endbulbs of Held/inhibitory synapses/bushy cells/Mover/

\section{Introduction}

Hearing relies on precise and rapid processing of auditory input. Sound reaches the cochlea, gets transferred to the brain stem and reaches the cortex. Along the way, signals get integrated and refined along a well-described, conserved pathway, called the binaural pathway. The first site of synaptic integration is the ventral cochlear nucleus (VCN; Young \& Oertel, 2003, 2010), where the auditory nerve fibers (ANFs), which receive their input from hair cells in the cochlea, synapse onto stellate and bushy cells (BCs), the latter of which will be in the focus of this study. BCs in the VCN are organized in a tonotopic manner, with cells coding low frequencies located in the ventral rostral part of the VCN, and cells coding high frequencies more located caudal and dorsal (Young \& Oertel, 2003). Myelinated type I AN fibers form big glutamatergic synapses onto BC somata, called endbulbs of Held. BCs also receive somatic inhibitory input, mainly from the dorsal cochlear nucleus (DCN).

BCs project to the ipsi- and contralateral superior olivary complex (Alibardi, 1998; Suneja et al., 1995). They target, among others, the principal cells of the medial nucleus of the trapezoid body (MNTB), with which they form the giant glutamatergic synapses known as the calyx of Held (Borst \& Soria van Hoeve, 2012). One characteristic of endbulb synaptic physiology is that it is highly plastic because of its strong depression (Yang \& Xu-Friedman, 2009). Additionally, endbulbs show a strong synaptic reliability, and are specialized to fire at high frequencies.

Synaptic processing both in the endbulbs of Held as well as the somatic inhibitory synapses relies on a highly complex machinery of - mostly - evolutionarily conserved proteins. A remarkably small number of presynaptic proteins can only be found in vertebrates, though. Among these are the AZ scaffolding proteins Bassoon and Piccolo, the motor adaptor Syntabulin, and the synaptic vesicle (SV) proteins Synuclein and Mover/SVAP30/TPRGL (Burré et al., 2006; Cai et al., 
2007; George, 2002; Gundelfinger et al., 2016; Thomas Kremer et al., 2007). Literature suggests that the main function of vertebrate-specific proteins is to increase the functional heterogeneity of synapses in the brain (Emes et al., 2008; Ryan \& Grant, 2009).

Mover is one of the very small number of vertebrate-specific proteins. It is a small phosphorprotein that is attached to SVs (Ahmed et al., 2013). Interestingly, its expression levels have been shown to be activity dependent (Kremer, 2008). We have recently described the heterogeneous distribution of the SV protein Mover in a quantitative manner (Rebecca Wallrafen \& Dresbach, 2018). We find a striking heterogeneity in the distribution on three distinct levels: across brain areas, within single brain areas and across synapse types. Knockdown of Mover at the calyx of Held, i.e. the synapse formed by BC axons onto principal cells of the MNTB, resulted in increased release probability and short-term depression (Körber et al., 2015). To test whether Mover might also influence synaptic input to BCs, we set out to characterize the distribution of Mover in synapses targeting BCs in the VCN and determine the structural effects of knocking out Mover.

\section{Results}

The vertebrate-specific protein Mover is heterogeneously expressed across the mouse brain (Rebecca Wallrafen \& Dresbach, 2018). To determine its effect on auditory processing, we first characterized the distribution of Mover in the VCN, where auditory nerve fibers terminate onto $B C s$, in detail.
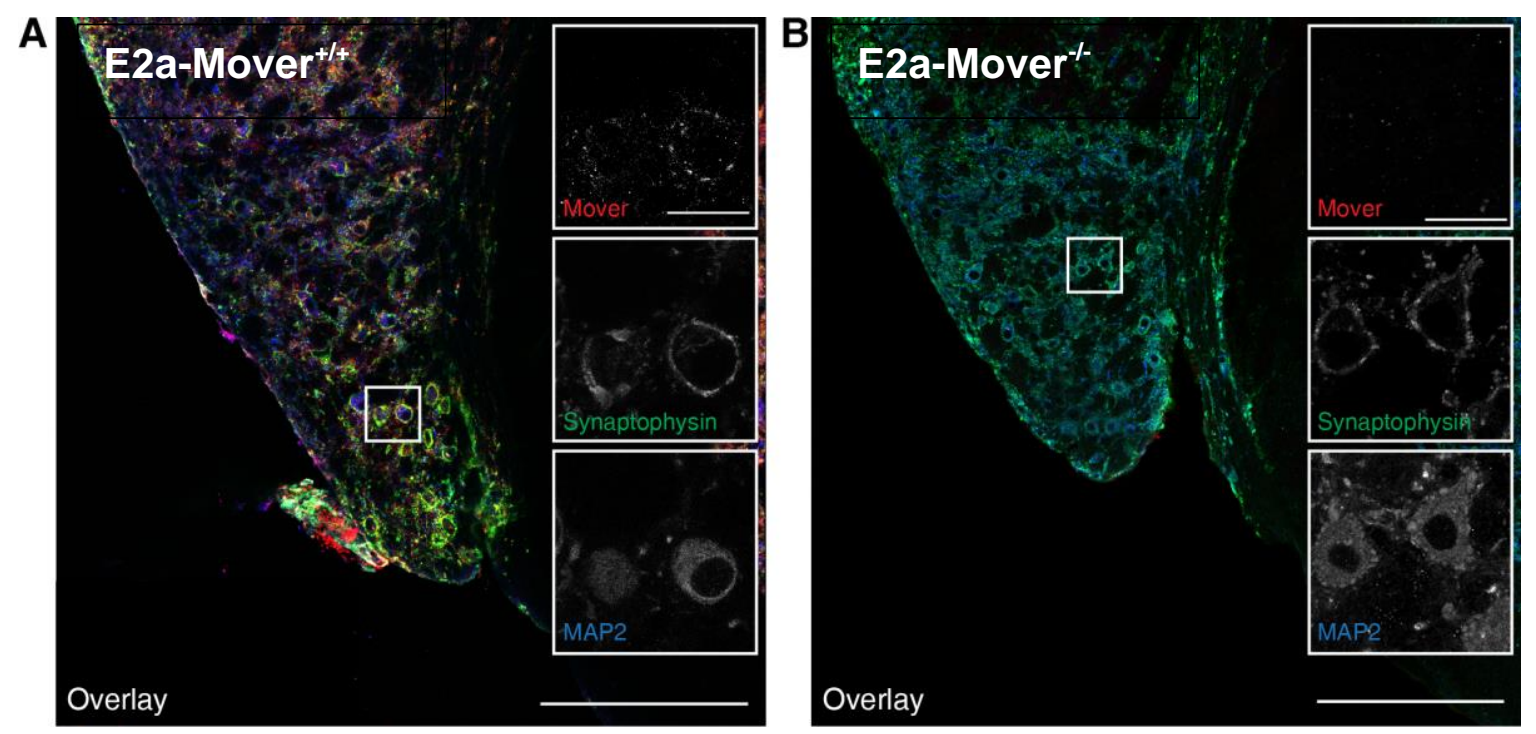

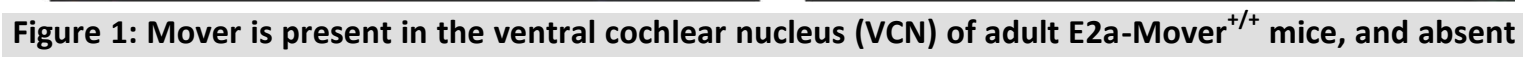

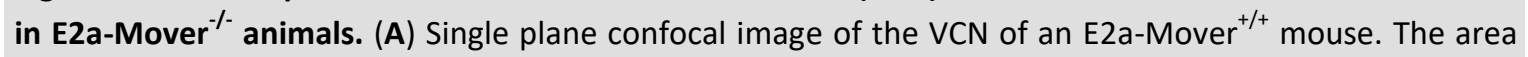
indicated by the white box is magnified in the panels on the right side of the image. Mover shows a punctate staining pattern (red in overview image, upper-most insert), similar to Synaptophysin (green, 
middle insert). MAP2 shows the bushy cell body (blue, lower insert). (B) Mover staining is absent in the VCN of the E2a-Mover ${ }^{--}$mouse. Scale bar $200 \mu \mathrm{m}$ in overview images, $20 \mu \mathrm{m}$ in magnification images.

Mover is present in the VCN of adult E2a-Mover ${ }^{+/+}$mice, and absent in E2a-Mover ${ }^{-/-}$animals.

We validated the presence of Mover in this brain region by immunofluorescence stainings using antibodies against Mover, the general synapse marker Synaptophysin and the somatodendritic marker microtubule-associated protein 2 (MAP2). We confirm that Mover is indeed present in the VCN of E2a-Mover ${ }^{+/+}$animals (Fig. 1A) and shows a punctate pattern at synaptic terminals surrounding $B C$ somata (magnification images on the right of Fig. 1A). The same triple staining in the E2a-Mover ${ }^{-/}$VCN revealed a normal synapse-staining of Synaptophysin, but no immunofluorescence for Mover (Fig. 1B).

\section{Mover colocalizes with presynaptic markers at inhibitory and excitatory synapses contacting} BCs.

Next, we set out to quantify the colocalization of Mover with Synaptophysin as a general synapse marker (Navone et al., 1986), the vesicular glutamate transporter 1 (vGluT1) as a marker for excitatory synapses (Ziegler et al., 2002), and the vesicular $\gamma$-aminobutyric acid (GABA) transporter (vGAT, Chaudhry et al., 1998) for inhibitory synapses (Fig. 2). We performed triple stainings for Mover, Synaptophysin and either vGlut1 (Fig. 2A) or vGAT (Fig 2B). On visual inspection, Mover nearly always colocalized with Synaptophysin. At vGAT-positive terminals, Mover intensity seemed brighter as compared to vGluT1-positive terminals. This visual impression was corroborated by the quantification of colocalization, where we employed Pearson's correlation analysis. While colocalization between Mover and vGluT1 was not particularly high (0.27, light grey, Fig. $2 \mathrm{C}$ ), colocalization with the inhibitory marker vGAT and Synaptophysin was significantly higher (0.48, medium grey, and 0.46 , dark grey, respectively; pvalues: Mover+vGluT1/Mover+vGAT: $10^{*} 10^{-12}$, Mover+vGluT1/Mover+Synaptophysin: $8 * 10^{-12}$, Fig. 2C). This indicates that Mover in enriched in inhibitory terminals, while it is also present at excitatory Endbulbs.

\section{Synaptic vesicles at Endbulbs of Held have more Mover per SV than inhibitory synapses contacting BCs.}

Upon visual inspection, we found higher Mover intensities at inhibitory terminals and the same seemed to be the case for the Synaptophysin intensity. We therefore quantified the intensities specifically at excitatory or inhibitory terminals, respectively. As background staining would 
influence the average fluorescence intensity across the image and thereby might falsify results, we used a thresholding approach. The vGluT1 (Fig. 3A) and vGAT (Fig. 3B) channel of the

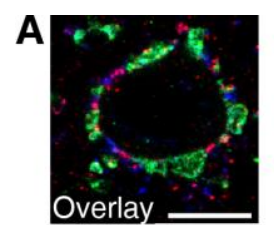

B

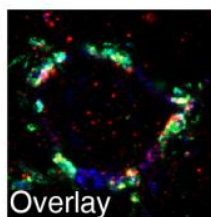

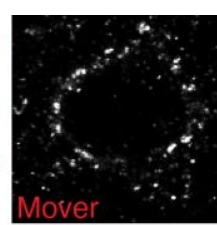

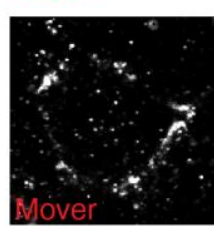

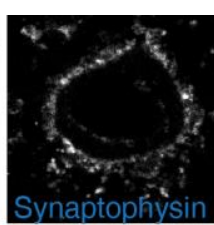

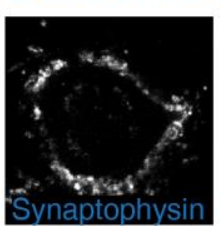

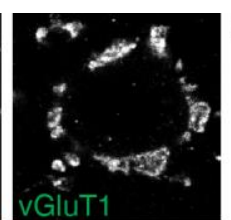

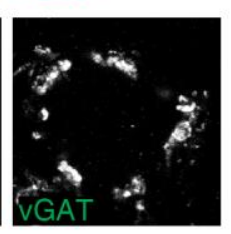

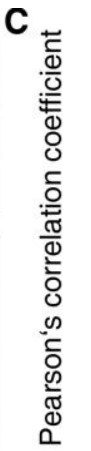

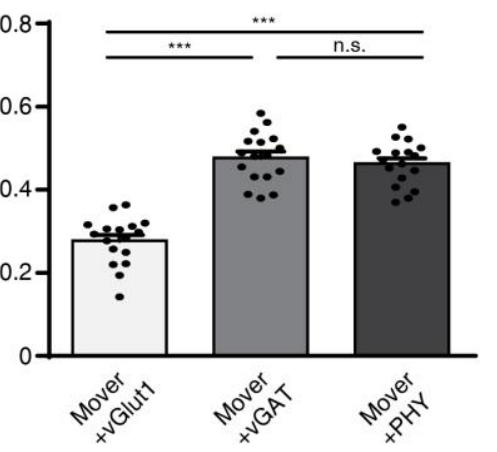

Figure 2: Mover colocalizes with presynaptic markers at synapses contacting bushy cells. (A) Single plane confocal image of a triple staining of Mover (left greyscale image), Synaptophysin (middle greyscale image) and vGluT1 (right greyscale image) at one bushy cell. The left-most panel shows the overlay of the three markers (Mover: red, Synaptophysin: blue, vGluT1: green). Note that many Mover-puncta do not colocalize with vGlut1, while the majority of vGluT1-puncta are Synaptophysin-positive. (B) Triple staining of Mover (left greyscale image), Synaptophysin (middle greyscale image) and VGAT (right greyscale image) at one bushy cell. The overlay-picture (Mover: red, Synaptophysin: blue, vGAT: green) shows higher Mover intensities at VGAT-positive synapses, all of which are also Synaptophysin-positive. (C) Quantification of the colocalization through Pearson's correlation coefficient. While colocalization between Mover and vGAT and Mover and Synaptophysin is equally high ( 0.48 and 0.46 , respectively; $p=0.46$ ) colocalization between Mover and vGluT1 is significantly lower (0.27, Mover+vGluT1/Mover+vGAT: $p=10^{*} 10^{-}$ ${ }^{12}$ Mover+vGluT1/Mover+Synaptophysin: $\mathrm{p}=8 * 10^{-12}$; Oneway ANOVA, multiple comparisons were corrected for with the Bonferroni method). Scalebar $10 \mu \mathrm{m}$, bars show mean \pm S.E.M., black dots represent single measurements. $N=3, n=18$.

respective triple staining was converted to a binary mask using a threshold. This threshold was arbitrarily chosen in a way that pixels that clearly belonged to a synaptic terminal where within threshold, while pixels that were obviously background staining were left out. The resulting binary mask was then applied to the Mover and Synaptophysin channel, so that pixels that were outside of the mask were black (i.e. intensity value 0), and pixels within the mask displayed the Mover or Synaptophysin staining. We then determined the average fluorescence intensity of Mover (Fig. 3C) and Synaptophysin (Fig. 3D) at excitatory and inhibitory terminals.

Both Mover and Synaptophysin were significantly more abundant in inhibitory terminals (Mover:

59.7 a.u. Fig. 3C, orange; Synaptophysin: 57.4 a.u., Fig. 3D, light blue) than in excitatory synapses

(Mover: 45.6 a.u., Fig. 3C, red; Synaptophysin: 38.1 a.u., Fig. 3D, dark blue; p-values Mover: 0.02, Synaptophysin: 0.004). This corroborated the visual impression. To determine the abundance of Mover relative to the number of SVs, i.e. the amount of Mover per SV, we calculated the ratio of 
Mover to Synaptophysin (Fig. 3E), working under the assumption that the Synaptophysin intensity scales proportionately to the number of vesicles. Surprisingly, excitatory terminals had higher Mover-to-Synaptophysin ratios (1.79, Fig. 3E, dark grey) than inhibitory terminals (1.38, Fig $3 E$, light grey, $p=0.04$ ), indicating that while inhibitory synapses targeting $B C s$ have higher absolute Mover values, endbulbs of Held have more Mover per SV.

To check whether BCs receive homogeneous input in respect to the Mover abundance in excitatory and inhibitory terminals, we performed a frequency distribution analysis of the Mover-to-Synaptophysin-ratio across all excitatory and inhibitory terminals at one $B C$, respectively (Fig. 3F). We compared the frequency distributions for the single BCs with each other (i.e. all excitatory or inhibitory inputs one $\mathrm{BC}$ receives, compared to another $\mathrm{BC}$ ), and did not find any differences, neither for excitatory terminals nor inhibitory ones. This suggests that the population of $B C$ inputs in regard to the Mover abundance per SV is homogeneous. We did, however, find differences between the average frequency distributions of excitatory (Fig. 3F, grey line) and inhibitory (Fig. 3F, black line) inputs. While both types of terminal showed a Gaussian distribution of the Mover-to-Synaptophysin ratio, the curve for inhibitory inputs was slightly shifted to the left, i.e. towards a lower Mover-to-Synaptophysin ratio. This corroborates the finding that endbulbs of Held have more Mover per SV than inhibitory synapses targeting BCs.

\section{Mover localizes more to the $A Z$ in inhibitory synapses.}

As a next step we decided to take a closer look at the subcellular distribution of Mover in both excitatory and inhibitory terminals. We performed triple stainings for Mover, vGluT1 or vGAT and Bassoon as a marker for the AZ. We employed our thresholding approach, using vGluT1 (Fig. 4A) or VGAT (Fig. 4B) as a binary mask and applied the mask to the Mover and Bassoon channels. We then used the Pearson's correlation analysis to determine the colocalization between Mover and 

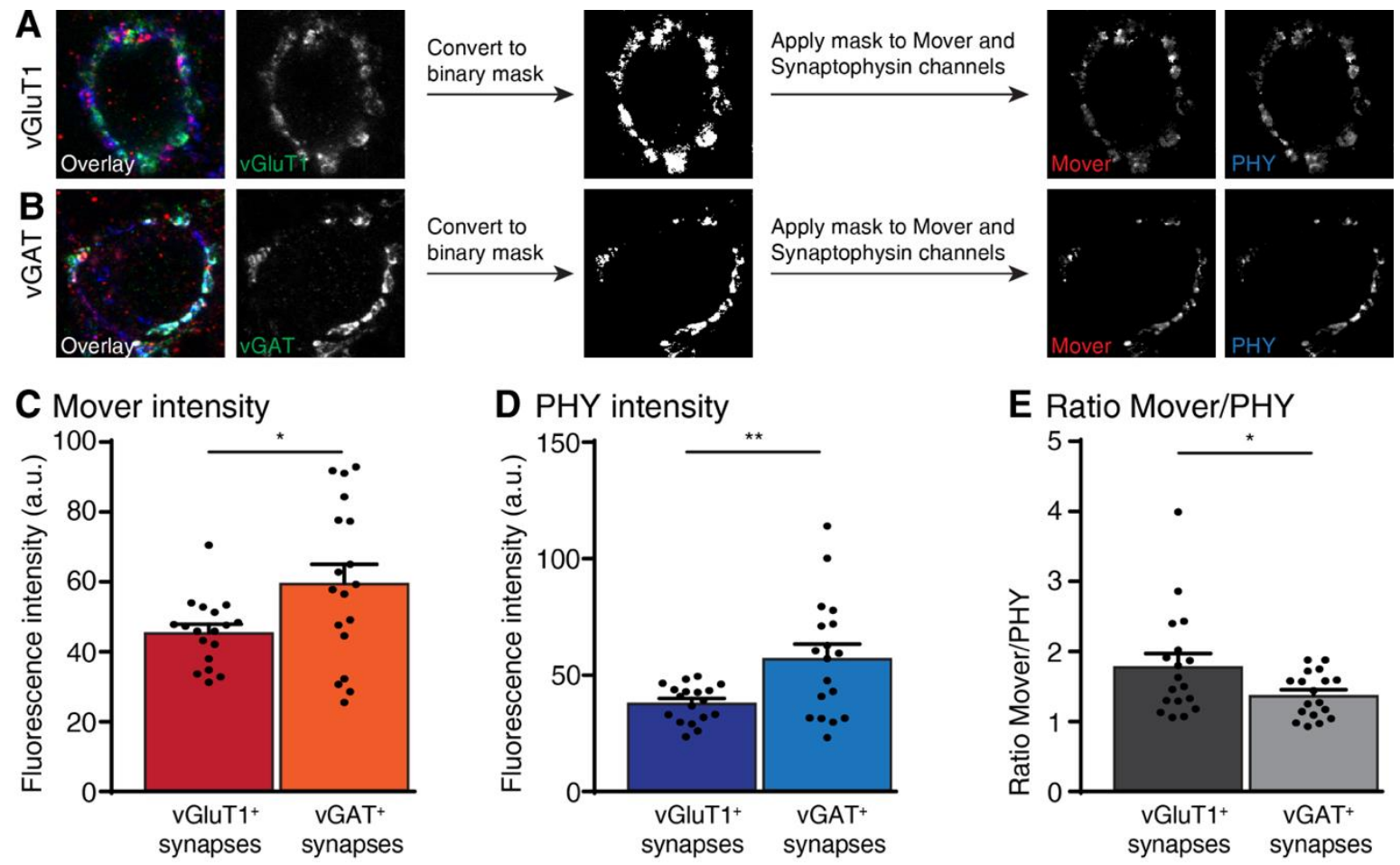

F Average frequency distribution of Mover/PHY ratio

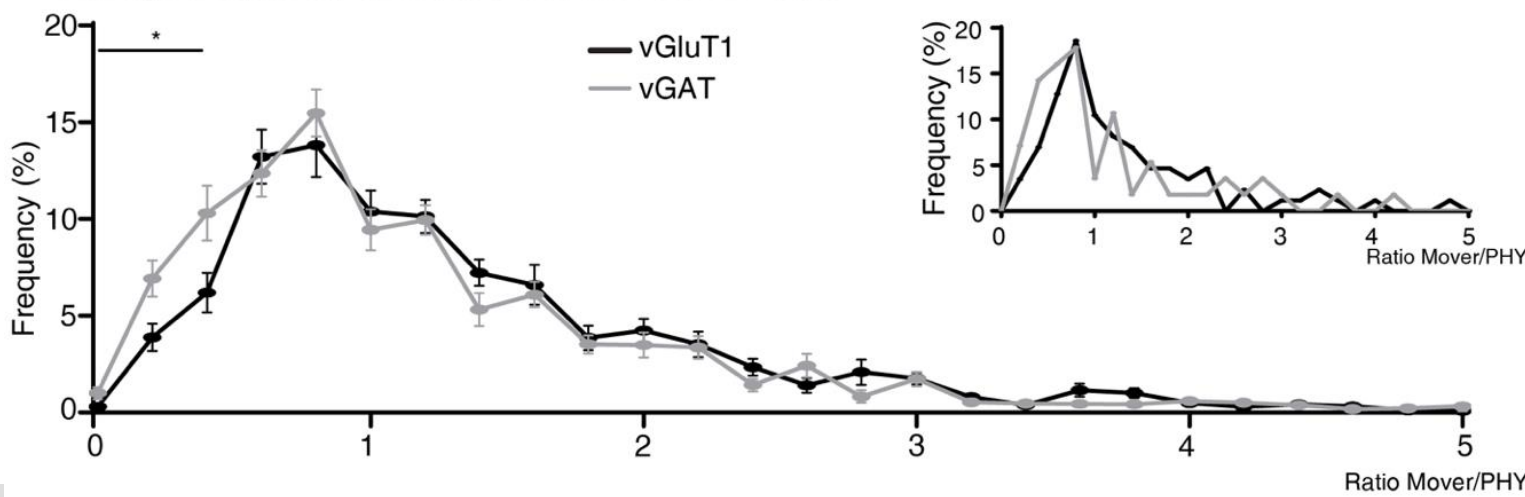

Figure 3: Synaptic vesicles at endbulbs of Held have more Mover per SV than inhibitory synapses contacting bushy cells. (A,B) Visualization of the quantification approach. Greyscale images of all vGluT1 (A)- or VGAT (B)-positive terminals targeting one bushy cell were subjected to thresholding and converted into a binary mask. This mask was then applied to the Mover and Synaptophysin channels of the same triple staining, respectively. This approach leaves only the staining of Mover and Synaptophysin in vGluT1/vGAT-positive areas. (C) After applying the mask, the absolute Mover fluorescence intensity (a.u.) was determined. These values were significantly higher at inhibitory synapses (59.7 a.u.) than at excitatory synapses (45.6 a.u.; $p=0.02$ ). The same was true for the Synaptophysin fluorescence intensity (D): values were higher at inhibitory synapses (57.4 a.u.) than at excitatory synapses (38.1 a.u.; $p=0.004)$. This indicates a higher abundance of synaptic vesicles (SVs) at inhibitory synapses. (E) The ratio of Mover to Synaptophysin, a measure of the amount of Mover relative to the number of SVs, is significantly higher in excitatory synapses (1.79) than in inhibitory synapses $(1.38, p=0.04)$, indicating that excitatory Endbulbs of Held have more Mover per SV. (F) Analysis of the average frequency distribution of the Mover-toSynaptophysin ratio across synapses targeting one single bushy cell reveals homogeneous populations of 
Fig. 3 (contd.): inhibitory and excitatory synapses. Of the inhibitory synapses, a significantly bigger portion shows low values of Mover to Synaptophysin (ratio 0-0.4, $p<0.05$, Student's $t$-test), corroborating results presented in (E). Main graph shows average values of 18 separate bushy cells. The grey line represents inhibitory synapses, the black line shows values for excitatory synapses. The insert shows a single example with the same color code. Bars in (C)-(E) show mean \pm S.E.M., black dots represent single measurements. Data points in (F) show mean \pm S.E.M. $N=3, n=18$.

Bassoon at excitatory (Fig. 4C, white) and inhibitory (Fig. 4C, grey) terminals. Upon visual inspection, Mover seemed to colocalize less frequently with Bassoon in excitatory synapses than in inhibitory ones, which was corroborated by the Pearson's analysis (colocalization in excitatory synapses: 0.142 ; inhibitory synapses: $0.238 ; p=0.007)$. Even though both values are fairly small, the difference between the localization of Mover to AZs in inhibitory versus excitatory synapses might indicate differential functions of Mover in the different terminals.
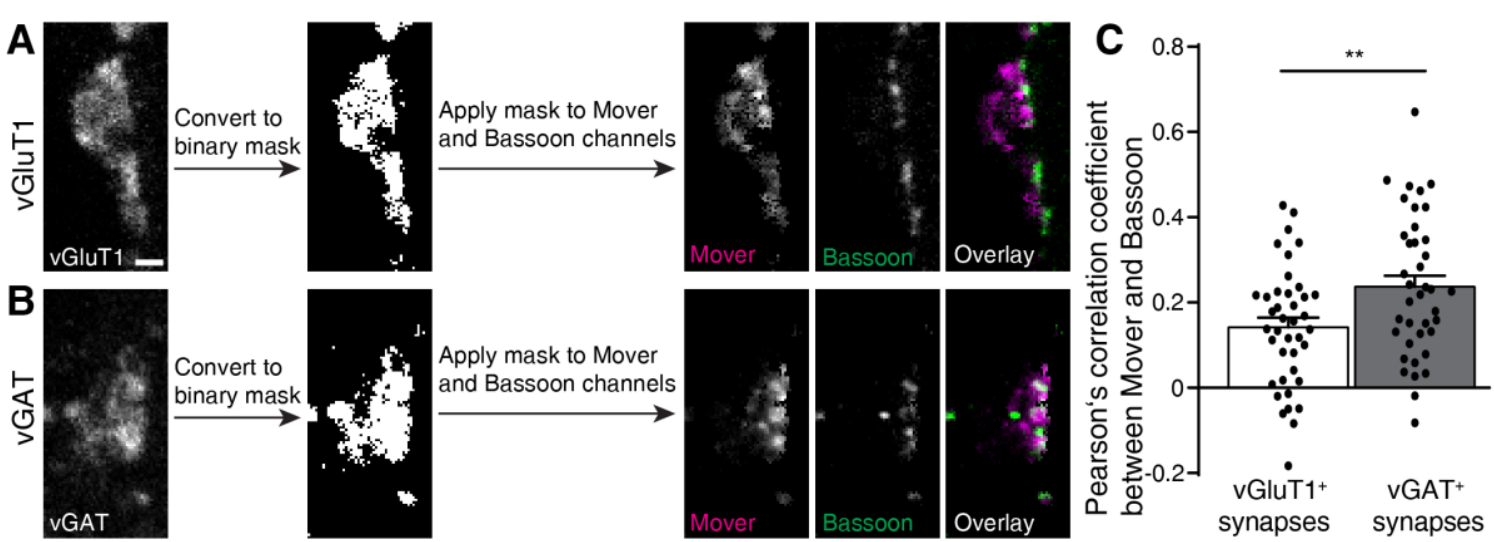

Figure 4: Mover localizes more to the AZs in inhibitory synapses. (A,B) Visualization of the quantification approach. Greyscale images of single vGluT1 (A)- or vGAT (B)-positive terminals were subjected to thresholding and converted into a binary mask. This mask was then applied to the Mover and Bassoon channels of the same triple staining, respectively. This approach leaves only the staining of Mover and Bassoon in vGluT1/vGAT-positive areas. (C) Quantification of the colocalization through Pearson's correlation coefficient. Colocalization between Mover and Bassoon was higher in inhibitory synapses (0.237) than in excitatory synapses $(0.142 ; p=0.007$; Student's $t$-test). Scalebar $=1 \mu \mathrm{m}$. Bars show mean \pm S.E.M., black dots represent single measurements. $N=3, n=36$.

\section{Discussion}

We investigated the distribution of the vertebrate-specific synaptic vesicle protein Mover in the VCN of the adult mouse brain. We find that Mover is present in both excitatory endbulbs of Held and inhibitory synapses targeting BCs. In the knock out model we use (Viotti et al., unpublished), Mover is completely absent from all synapses, which we confirm by immunofluorescence. Structural investigation of excitatory and inhibitory synapses targeting BCs reveals a differential 
distribution of Mover across the synapse types in the wt condition and distinct effects in KO synapses.

\section{Mover is differentially expressed in inhibitory and excitatory synapses targeting BCs.}

Immunofluorescent stainings revealed that Mover is present in both excitatory and inhibitory synapses targeting BCs in the VCN. Previous research from our lab has shown that the distribution of Mover throughout the mouse brain and across synapse types is unusually heterogeneous, and that its presence at both synapse types is not a given: in the hippocampus, Mover is only present at excitatory synapses involved in intrahippocampal computation (Rebecca Wallrafen \& Dresbach, 2018).

In the cerebellum, however, Mover was found only at inhibitory synapses in the cerebellar glomeruli in the granular cell layer (Kremer et al., 2007), while it was present at both excitatory and inhibitory synapses in the medioposterior nucleus of the amygdala (Rebecca Wallrafen \& Dresbach, 2018). This differential distribution suggests a role for Mover in increasing the functional diversity of brain areas by specifically modulating certain subsets of synapses. 
We find that absolute expression levels of Mover are higher in inhibitory synapses than in endbulbs. However, we also find more SVs at inhibitory synapses than excitatory endbulbs. We therefore calculated the ratio of Mover to Synaptophysin to determine the amount of Mover relative to the number of SVs. With this calculation we find that endbulbs have more Mover per SV than inhibitory synapses, in spite of the lower absolute levels.

Mover might function as an adapter between Bassoon and SVs at the endbulb of Held.

Mover, which consists of 266 amino acids and weighs $26 \mathrm{kDA}$, was initially described as a binding partner of the AZ scaffolding molecule Bassoon (Kremer et al., 2007). Bassoon is a 420kDA protein that shows a stretched conformation at the synaptic terminal, with the $\mathrm{N}$-terminus closer to the presynaptic membrane, and the C-terminus sticking into the synaptic bouton (Dani et al., 2010; Gundelfinger \& Fejtova, 2012; Gundelfinger et al., 2016; Ivanova et al., 2015; Limbach et al., 2011; Sanmartí-Vila et al., 2000). The putative interaction site of Mover and Bassoon lies in the C-terminus of Bassoon, i.e. further away from the synaptic cleft. The antibody against Bassoon used in this study was raised against amino acids 756-1001 (of a total of 3938 aa). Low colocalization values, as we find them in our study, are therefore not surprising and can be explained by the fact that the epitopes in the Bassoon and Mover molecules are just too far apart to show high Pearson's correlation values. Super-resolution microscopy using nanobodies directed against Mover and different epitopes in the Bassoon molecule should yield information on the precise binding site of Mover to Bassoon.

\section{Mover might be involved in SV docking in inhibitory synapses}

At inhibitory terminals, things look different. While we find higher absolute Mover levels, the ratio of Mover to Synaptophysin fluorescence intensity is lower, indicating lower levels of Mover per SV. This in itself does not mean much, but it adds another level of heterogeneity, which we always encounter when studying Mover. While Bassoon disruption resulted in downregulation of Mover at excitatory synapses, Mover levels in inhibitory synapses targeting BCs remain unchanged (Mendoza Schulz et al., 2014). On first glance this seems contradictory to our results, showing higher colocalization between Mover and Bassoon in inhibitory synapses. On second glance, it allows for the speculation of a Bassoon-independent Mover-function in inhibitory synapses. Further, inhibitory synapse-specific studies, should reveal whether this hypothesis can be confirmed.

\section{Input to BCs is homogeneous in respect to Mover levels.}

Endbulbs are highly plastic synapses, displaying short-term depression (Yang \& Xu-Friedman, 2009). Hitherto it remains unclear what the functional purpose of the depression is, and how it 
influences information processing. A study using voltage-clamp recordings showed that endbulbs were rather heterogeneous regarding their plasticity: the amount of depression varied significantly between endbulbs, while sibling endbulbs (i.e. endbulbs originating from the same ANF) displayed similar depression strengths (Yang \& Xu-Friedman, 2009). As Mover is believed to influence synaptic plasticity and (depression) strength, we investigated whether the overall input to one $\mathrm{BC}$ is homogeneous in regard to the amount of Mover per SV. We therefore binned the Mover-to-Synaptophysin ratio and determined the frequency distribution across all endbulbs or inhibitory synapses contacting one BC. Interestingly, we found that input to BCs is indeed homogeneous, i.e. that the overall input to one BC does not differ significantly from other BCs. This was true for both excitatory and inhibitory inputs to BCs. While this analysis tells us that the overall input is the same for every $B C$, it does not reveal whether single endbulbs differ from each other in respect to their relative Mover levels. Further studies analyzing single endbulbs terminating onto one $B C$, potentially combined with tracing studies to determine ANF origin, should shed light on the question whether differences in depression in single endbulbs can potentially be explained by heterogeneous Mover levels present in these endbulbs.

\section{Materials and Methods}

\section{Experimental animals}

No experiments involving live animals were conducted for this study. Experiments involving euthanizing of animals to obtain brain samples were approved by the local animal protection authorities (Tierschutzkommission der Universitätsmedizin Göttingen) under the approval number T $10 / 30$.

\section{Immunofluorescence staining}

For immunofluorescence stainings, three adult male E2a-Mover ${ }^{+/+}(w t)$ and three adult male E2a-Mover ${ }^{-1-}$ mice were euthanized by $\mathrm{CO}_{2}$ inhalation and transcardially perfused with $0.9 \%$ saline followed by perfusion with $4 \%$ paraformaldehyde (PFA) in $0.1 \mathrm{M}$ phosphate buffer (PB), $\mathrm{pH}$ 7.4. Brains were removed and postfixed in $4 \% \mathrm{PFA}$ in $0.1 \mathrm{M}$ PB for 24 hours at $4^{\circ} \mathrm{C}$.

Brains were cut into $40 \mu \mathrm{m}$ thick coronal sections using a vibrating microtome. Sections were collected in $0.1 \mathrm{M} \mathrm{PB}$ and stored at $4^{\circ} \mathrm{C}$ until further use. Sections containing the VCN were selected according to the mouse brain atlas (Paxinos \& Franklin, 2001), and per antibody combination, three slices per brain containing the VCN were stained. Free floating sections were rinsed with PB once and blocked with 2.5\% goat serum (Merck KGaA, Darmstadt, Germany), 2.5\% donkey serum (Merck Chemikals GmbH, Darmstadt, Germany), 1\% Triton X-100 in PB for 3 hours at room temperature (RT). The following relevant primary antibodies were applied 
overnight at $4^{\circ} \mathrm{C}$ : Mover (1:1000 rabbit anti-Mover polyclonal, Cat. No. 248003, RRID: AB_10804285, Synaptic Systems, Germany), Synaptophysin 1 (1:1000 mouse anti-Synaptophysin monoclonal, Cat. No. 101011, RRID: AB_887824, Synaptic Systems, Germany), MAP2 (1:2000 gp anti-MAP2 polyclonal, Cat. No. 188004, Synaptic Systems, Germany), vGlut1 (1:5000 guinea pig anti-vGluT1 polyclonal, Cat. No. 135304, RRID: AB_887878, Synaptic Systems, Germany), vGAT (1:500 chicken anti-vGAT polyclonal, Cat. No. 131006, RRID: AB_2619820, Synaptic Systems, Germany) and Bassoon (1:2000 mouse anti-Bassoon monoclonal, clone SAP7F407, Cat. No. ADIVAM-PS003, RRID: AB_10618753, Enzo Life Sciences, USA). Sections were washed with 2\% goat serum in PB and incubated with relevant secondary antibodies for 90 min at RT in the dark: donkey anti-rabbit 647 (1:2000, Alexa Fluor, Invitrogen, USA), goat anti-guinea pig Cy2 (1:1000), goat anti-chicken Cy3 (1:1000), donkey anti-mouse Cy3 (1:2000, Jackson ImmunoResearch, USA; all antibodies were diluted in $0.5 \%$ goat serum, $0.5 \%$ donkey serum, $0.2 \%$ Triton $\mathrm{X}-100$ in PB). Sections were washed with $1 \%$ goat serum in PB, incubated with 4',6-diamidino-2-phenylindole (DAPI; 1:1000 in PB) for $5 \mathrm{~min}$, rinsed again and mounted on Menzel microscope slides. To ensure minimal variability, brains from all animals were cut, stained and treated simultaneously.

\section{Microscopy}

All images were acquired using a Zeiss LSM800 confocal microscope, running the ZEN blue software (version 2.3, Zeiss, Oberkochen, Germany). Laser settings were adjusted so that few pixels were overexposed to ensure maximum distribution of grey values.

For overview images, triple stainings for Mover, MAP2 and Synaptophysin were performed and virtual tissues composed of single tiles (1024x1024 px, moderate scan speed, 4-times averaging) were acquired using a 20x objective (air immersion, NA x). Using the corresponding functions of the program, virtual tissues were stitched and exported as TIFF-files.

To determine the colocalization of Mover with vGluT1, vGAT, Synaptophysin and Bassoon, triple stainings were performed and single images were acquired using a $63 x$ objective (oil immersion, NA 1.4. No adjustments for brightness or contrast were made, and images were exported as TIFF-files.

\section{Colocalization analysis}

To analyze the colocalization of Mover with other synaptic markers, we subtracted the background staining from the monochromatic image using the "Subtract Background" function of FIJI (Rolling ball radius: 50 pixels for all channels). We then used the "Colocalization Test" plugin to determine the Pearson's correlation coefficient between Mover and vGluT1, between Mover and vGAT and vGluT1 and vGAT as a means of control (for more information on Pearson's 
correlation see Adler \& Parmryd, 2010 and Dunn, Kamocka, \& McDonald, 2011). To verify that no random colocalization was measured, we rotated one of the images by $90^{\circ}$ and analyzed colocalization: doing this, no colocalization was observed, and Pearson's correlation values were very low to negative. Values were plotted in scatter plots including bar charts (average \pm S.E.M). For statistical testing, a Oneway ANOVA with Bonferroni correction for multiple comparisons was performed using GraphPrism 6.

\section{Synapse-specific analysis}

To analyze the abundance of Mover and Synaptophysin and the colocalization between Mover and Bassoon in excitatory and inhibitory terminals separately, we used the vGluT1 and VGAT staining as masks. For that, we applied an arbitrary threshold to the greyscale vGluT1 and vGATchannels, respectively. The threshold was chosen in a way so that pixels that clearly belonged to a synaptic terminal where within threshold, while pixels that were obviously background staining were left out. The images were converted into a binary mask and applied to the Mover and Synaptophysin (fig. 3) and the Mover and Bassoon channels (fig. 4), respectively. In the resulting single-channel images (showing the Mover/Synaptophysin/Bassoon intensities at vGluT1 or vGAT-positive pixels), we determined the average fluorescence intensity of Mover and Synaptophysin, and the colocalization of Mover and Bassoon (for detailed explanation see "Colocalization analysis"). The ratio of Mover to Synaptophysin (fig. 3E) was determined (as previously described in Wallrafen \& Dresbach, 2018) by dividing the average fluorescence intensity in the Mover channel of one single image by that in the Synaptophysin channel. Values were plotted in scatter plots including bar charts (average \pm S.E.M). For statistical testing, a Student's $t$-test was performed using GraphPrism 6.

To determine the population homogeneity of excitatory and inhibitory inputs to one $B C$ in respect to the Mover abundance relative to the number of SVs, we performed a frequency distribution analysis of the Mover-to-Synaptophysin-ratio across all excitatory and inhibitory terminals at one $B C$, respectively. In images showing only one $B C$, we determined the ratio for every vGluT1 and VGAT-positive pixel separately and analyzed the frequency distribution with GraphPrism 6. We binned the values in 0.2-step sizes and displayed the single data points (average \pm S.E.M) in a line graph. Data points for the same bin sizes between vGluT1 and vGATpositive terminals were compared using a Student's $t$-test.

\section{Antibody specificity}

To verify the absence of non-specific immunostaining using the immunofluorescence method, primary antibodies were excluded but the secondary antibody steps were performed to 
completion. Under these conditions, no cross-reactivity or significant background staining was observed (not shown).

\section{Acknowledgements}

We thank Irmgard Weiß for excellent technical assistance. Hermes Pofantis for valuable input regarding the auditory system. Asha Akula for KO mouse.

\section{Author contributions}

RW performed immunofluorescence experiments, analyzed data and wrote manuscript. TD conceived immunofluorescence experiments (partly with RW) and prepared the manuscript.

\section{Conflict of interest}

The authors declare that the research was conducted in the absence of any commercial or financial relationships that could be construed as a potential conflict of interest.

\section{References}

Adler, J., \& Parmryd, I. (2010). Quantifying colocalization by correlation: The pearson correlation coefficient is superior to the Mander's overlap coefficient. Cytometry Part A, 77(8), 733742.

Alibardi, L. (1998). Ultrastructural and immunocytochemical characterization of neurons in the rat ventral cochlear nucleus projecting to the inferior colliculus. Annals of Anatomy, 180(5), 415-426. http://doi.org/10.1016/S0940-9602(98)80102-7

Borst, J. G. G., \& Soria van Hoeve, J. (2012). The Calyx of Held Synapse: From Model Synapse to Auditory Relay. Annual Review of Physiology, 74(1), 199-224. http://doi.org/10.1146/annurev-physiol-020911-153236

Burré, J., Beckhaus, T., Corvey, C., Karas, M., Zimmermann, H., \& Volknandt, W. (2006). Synaptic vesicle proteins under conditions of rest and activation: Analysis by 2-D difference gel electrophoresis. Electrophoresis, 27(17), 3488-3496.

Cai, Q., Pan, P.-Y., \& Sheng, Z.-H. (2007). Syntabulin-Kinesin-1 Family Member 5B-Mediated Axonal Transport Contributes to Activity-Dependent Presynaptic Assembly. Journal of Neuroscience, 27(27), 7284-7296.

Chaudhry, F. a., Reimer, R. J., Bellocchio, E. E., Danbolt, N. C., Osen, K. K., Edwards, R. H., \& Storm-Mathisen, J. (1998). The vesicular GABA transporter, VGAT, localizes to synaptic vesicles in sets of glycinergic as well as GABAergic neurons. Journal of Neuroscience, 18(23), 9733-9750. 
Dani, A., Huang, B., Bergan, J., Dulac, C., \& Zhuang, X. (2010). Superresolution Imaging of Chemical Synapses in the Brain. Neuron, 68(5), 843-856. http://doi.org/10.1016/j.neuron.2010.11.021

Dunn, K. W., Kamocka, M. M., \& McDonald, J. H. (2011). A practical guide to evaluating colocalization in biological microscopy. Am J Physiol Cell Physiol, 300, C723-C742.

Emes, R. D., Pocklington, A. J., Anderson, C. N. G., \& Bayes, A. (2008). Evolutionary expansion and anatomical specialization of synapse proteome complexity. Nat Neuroscience,11(7), 799-806.

George, J. M. (2002). The synucleins.Genome Biology, 3(1), 1-6.

Gundelfinger, E. D., \& Fejtova, A. (2012). Molecular organization and plasticity of the cytomatrix at the active zone. Current Opinion in Neurobiology, 22(3), 423-430. http://doi.org/10.1016/j.conb.2011.10.005

Gundelfinger, E. D., Reissner, C., \& Garner, C. C. (2016). Role of Bassoon and Piccolo in Assembly and Molecular Organization of the Active Zone. Frontiers in Synaptic Neuroscience, 7(1).

Ivanova, D., Dirks, A., Montenegro-venegas, C., Schöne, C., Altrock, W. D., Marini, C., ... Fejtova, A. (2015). Synaptic activity controls localization and function of CtBP 1 via binding to Bassoon and Piccolo, 34(8).

Jahn, R., \& Südhof, T. C. (1994). Synaptic Vesicles and Exocytosis. Annual Review of Neuroscience, 17, 219-46.

Jean, P., de la Morena, D. L., Michanski, S., Tobón, L. M. J., Chakrabarti, R., Picher, M. M., ... Moser, T. (2018). The synaptic ribbon is critical for sound encoding at high rates and with temporal precision. ELife, 7, 1-39. http://doi.org/10.7554/eLife.29275

Jung, S., Oshima-Takago, T., Chakrabarti, R., Wong, A. B., Jing, Z., Yamanbaeva, G., ... Moser, T. (2015). Rab3-interacting molecules $2 \alpha$ and $2 \beta$ promote the abundance of voltage-gated $\mathrm{Ca}$ $\checkmark 1.3 \mathrm{Ca}{ }^{2+}$ channels at hair cell active zones. Proceedings of the National Academy of Sciences, 112(24), E3141-E3149. http://doi.org/10.1073/pnas.1417207112

Karlsson, U., \& Schultz, R. (1965). Fixation of the Central Nervous System from Electron Microscopy by Aldehyde Perfusion. I. Preservation with Aldehyde Perfusates versus direct Perfusion with Osmium Tetroxide with special Reference to Membranes and the Extracellular Space. Journal of Ultrastructure Research, Feb(12), 160-86.

Körber, C., Horstmann, H., Venkataramani, V., Herrmannsdörfer, F., Kremer, T., Kaiser, M., ... Kuner, T. (2015). Modulation of Presynaptic Release Probability by the Vertebrate-Specific Protein Mover. Neuron, 87(3), 521-533. 
Kremer, J. R., Mastronarde, D. N., \& McIntosh, J. R. (1996). Computer visualization of threedimensional image data using IMOD. Journal of Structural Biology, 116(1), 71-76. http://doi.org/10.1006/jsbi.1996.0013

Kremer, T., Kempf, C., Wittenmayer, N., Nawrotzki, R., Kuner, T., Kirsch, J., \& Dresbach, T. (2007). Mover is a novel vertebrate-specific presynaptic protein with differential distribution at subsets of CNS synapses. FEBS Letters, 581(24), 4727-4733.

Limbach, C., Laue, M. M., Wang, X., Hu, B., Thiede, N., Hultqvist, G., \& Kilimann, M. W. (2011). Molecular in situ topology of Aczonin/Piccolo and associated proteins at the mammalian neurotransmitter release site. Proceedings of the National Academy of Sciences, 108(31), E392-E401. http://doi.org/10.1073/pnas.1101707108

Mastronarde, D. N. (2005). Automated electron microscope tomography using robust prediction of specimen movements. Journal of Structural Biology, 152(1), 36-51. http://doi.org/10.1016/j.jsb.2005.07.007

Mendoza Schulz, A., Jing, Z., María Sánchez Caro, J., Wetzel, F., Dresbach, T., Strenzke, N., ... Moser, T. (2014). Bassoon-disruption slows vesicle replenishment and induces homeostatic plasticity at a CNS synapse. EMBO Journal, 33(5), 512-527.

Navone, F., Jahn, R., Gioia, G. Di, Stukenbrok, H., Greengard, P., \& Camilli, P. De. (1986). Protein p38: An integral membrane protein specific for small vesicles of neurons and neuroendocrine cells. Journal of Cell Biology, 103(6), 2511-2527.

Paxinos, G., \& Franklin, K. B. J. (2001). The Mouse Brain in Stereotaxic Coordinates(2nd editio). San Diego: ACADEMIC PRESS.

Ryan, T. J., \& Grant, S. G. N. (2009). The origin and evolution of synapses. Nature Reviews Neuroscience,10(11), 701-712.

Sanmartí-Vila, L., Tom Dieck, S., Richter, K., Altrock, W., Zhang, L., Volknandt, W., ... Dresbach, T. (2000). Membrane association of presynaptic cytomatrix protein Bassoon. Biochemical and Biophysical Research Communications, 275(1), 43-46. http://doi.org/10.1006/bbrc.2000.3256

Suneja, S. K., Benson, C. G., Gross, J., \& Potashner, S. J. (1995). Evidence for Glutamatergic Projections from the Cochlear Nucleus to the Superior Olive and the Ventral Nucleus of the Lateral Lemniscus. Journal of Neurochemistry, 64(1), 161-171. http://doi.org/10.1046/j.1471-4159.1995.64010161.x

Wallrafen, R., \& Dresbach, T. (2018). The Presynaptic Protein Mover Is Differentially Expressed Across Brain Areas and Synapse Types. Frontiers in Neuroanatomy, 12, 58. http://doi.org/10.3389/fnana.2018.00058 
Wong, A. B., Rutherford, M. A., Gabrielaitis, M., Pangršič, T., Göttfert, F., Frank, T., ... Moser, T. (2014). Developmental refinement of hair cell synapses tightens the coupling of Ca2+influx to exocytosis. EMBO Journal, 33(3), 247-264. http://doi.org/10.1002/embj.201387110

Yang, H., \& Xu-Friedman, M. A. (2009). Impact of Synaptic Depression on Spike Timing at the Endbulb of Held. Journal of Neurophysiology, 102(3), 1699-1710. http://doi.org/10.1152/jn.00072.2009

Young, E. D., \& Oertel, D. (2003). Cochlear nucleus. In G. M. Shepherd (Ed.), The synaptic organization of the brain(pp. 125-163). New York: Oxford University Press.

Young, E. D., \& Oertel, D. (2010). Cochlear nucleus. In G. M. Shepherd \& S. Grillner (Eds.), Handbook of brain microcircuits(pp. 215-223). New York: Oxford University Press.

Ziegler, D. R., Cullinan, W. E., \& Herman, J. P. (2002). Distribution of vesicular glutamate transporter mRNA in rat hypothalamus. Journal of Comparative Neurology, 448(3), 217229. 


\section{DISCUSSION}

We first established an immunofluorescence approach, allowing for the quantitative description of the distribution of Mover relative to the number of synaptic vesicles (SVs) in a certain brain area. We compare the ratio in one brain area to that across the whole hemisphere, to compensate for the inherent variability of immunofluorescent stainings. This approach allows for a quantitative description of the Mover abundance relative to the number of SVs in a certain brain area and even within. Hitherto, quantitative studies generally relied on biochemical methods such as western blotting and mass spectrometry (Charette et al., 2010; Heidebrecht et al., 2009; Taylor et al., 2013; Toki et al., 2017) rather than immunofluorescence. In some cases, several methods are used to complement each other (i.e. Wilhelm et al., 2014). We describe for the first time an immunofluorescence approach that can be employed without the need for biochemical methods, which yields certain advantages: the protein of interest is detected in its physiological surroundings, allowing for a combination of quantification analysis and (subcellular) localization.

In our first study, we employ this quantitative immunofluorescence approach to show that the Mover distribution is unusually heterogeneous on three levels: (1) Mover is differentially expressed across different brain areas. Some areas, such as the primary motor cortex or the granular layer of the cerebellum have low levels of Mover relative to Synaptophysin, while other areas, such as the ventral pallidum, molecular layer of the cerebellum and amygdala show high levels of Mover expression. (2) The Mover expression within single brain areas can be quite heterogeneous as well: in the hippocampus, for example, Mover is most abundant in regions involved in intrahippocampal computation, while expression levels are low in the main input and output regions. (3) Even between synapse types, the distribution of Mover is by no means homogeneous: in the hippocampus, Mover is exclusively associated with excitatory synapses. In the different amygdaloid nuclei, Mover is differentially associated with either only excitatory synapses, such as in the basal and lateral nuclei, while it is present at both types of synapses in the medial nucleus.

In the second manuscript we describe Mover at the first relay station of the auditory pathway, i.e. in the ventral cochlear nucleus (VCN). We find that also there, Mover is differentially expressed across excitatory and inhibitory synapses targeting busyh cells (BCs). Through structural analysis of Mover wild type synapses compared to a complete knockout of Mover we 
find that Mover has differential effects on SV-to-active-zone-distances, also called coupling distance, in excitatory and inhibitory synapses. Based on these findings we propose two distinct working mechanisms for Mover, which will be discussed in chapter 5.3.

\subsection{MOVER IS A VERTEBRATE-SPECIFIC PRESYNAPTIC PROTEIN}

While most of the proteins of the core machinery mediating presynaptic processes are evolutionarily conserved, a remarkably small number occurs only in vertebrates. So far, only a handful of vertebrate-specific presynaptic proteins have been discovered: Bassoon, Piccolo, Synuclein, Doc2 and Mover.

Bassoon and Piccolo are multi-domain scaffolding molecules that are part of the CAZ. They are thought to stabilize vertebrate synapses, as double-knockdown of these two proteins leads to the disassembly of synapses (Waites et al., 2013). Interestingly, when only knocking out Piccolo, the phenotype is rather mild, suggesting that the functions of Bassoon and Piccolo are, at least to some extent, redundant (Mukherjee et al., 2010).

Like with Bassoon and Piccolo, the physiological function of Synuclein remains largely unknown. Mutations in the $\alpha$-Synuclein gene, however, have been described to be causative of early-onset familial Parkinson's disease (Goedert, 2001; Mezey et al., 1998). Protein aggregates of Synuclein have also been described in other neurodegenerative diseases, such as Alzheimer's and Lewy body disease.

In contrast, the function of the vertebrate-specific presynaptic protein Doc2 has been described: it supports spontaneous synaptic transmission (also called mini release; Groffen et al., 2010; Pang et al., 2011). Whether it does so in a $\mathrm{Ca}^{2+}$ - dependent or -independent manner is unclear though.

In general, the main function of vertebrate-specific proteins is believed to increase the functional heterogeneity of the brain (Emes et al., 2008; Ryan \& Grant, 2009). Interestingly, although most of the functions remain undiscovered, they seem to be involved in different processes, ranging from stabilization of synapses to mediating $\mathrm{Ca}^{2+}$-independent mini release. Mutations in these proteins seem to be associated with neurodegenerative and psychological disorders.

The fact that Mover is so heterogeneously distributed throughout the brain and across synapses types suggests that its modulatory function is even more specialized and applies to only a subset of synapses. Interestingly, Mover has also been associated with psychological disorders: in 
postmortem brains of human schizophrenic patients (Clark et al., 2006), Mover was strongly upregulated, while mice that globally lack Mover show reduced anxiety levels (Viotti et al., unpublished). Taken together, the involvement of vertebrate-specific proteins in neurodegeneration make them an interesting target for brain research.

\subsection{MOVER IS HETEROGENEOUSLY EXPRESSED ACROSS BRAIN AREAS}

We applied our quantification approach to 16 brain areas in the adult mouse brain. We show that Mover is heterogeneously expressed across these areas. Mover is particularly abundant in the ventral pallidum, the septal nuclei and the amygdala. The former two regions have both been associated with addiction and reward behavior. High levels of Mover in these regions suggest that these processes might be influenced by the presence or absence of Mover, and that such behaviors might be altered in the Mover KO mouse model.

In the amygdala, we detected high levels of Mover throughout the different nuclei. Interestingly, our Mover KO mouse model shows an altered anxiety-related phenotype. Details of this phenotype and putative Mover involvement will be discussed below.

The absence of Mover could also have functional implications. In the mouse brain we find Mover levels below average the anterior cingulate cortex (ACC). In human schizophrenic patients, however, Mover is upregulated in the ACC (Clark et al., 2006). Therefore, not only a lack of Mover (in regions that have higher Mover expression under physiological conditions) can have behavioral effects, but also the increased presence of Mover (in regions where it should be absent) might modulate and influence normal brain function.

Overall, the heterogeneous Mover distribution suggests that Mover might provide specialization to brain regions in a differential manner, thereby contributing to the functional diversity of brain areas.

\subsection{MOVER CAN BE DIFFERENTIALLY EXPRESSED WITHIN BRAIN AREAS}

In addition to its heterogeneous distribution across different brain areas, Mover also shows a striking heterogeneity within single areas. In some regions, such as the hippocampus and the somatosensory cortex, Mover is heterogeneously distributed, with layers with high Mover levels and layers with Mover levels below detection limit. In other regions, such as the amygdala, Mover is homogeneously abundant, despite the heterogeneity of the amygdaloid nuclei. In the following, I will discuss the heterogeneous distribution of Mover within single brain regions, 
focusing on the three aforementioned areas: the hippocampus and the somatosensory cortex as examples for brain regions with heterogeneous Mover levels, and the amygdala as an example for a region with homogeneous Mover expression levels.

\subsubsection{Mover is heterogeneously expressed within the Hippocampus}

Within the hippocampus, we find a striking heterogeneity in the Mover distribution across the different layers. We find low Mover relative to Synaptophysin in all cell body layers of the hippocampus, i.e. in the granular layer of the DG and the pyramidal cell layers of CA3 and CA1. In these layers, mainly inhibitory synapses target the cell bodies of the pyramidal and granule cells. Low Mover levels in these layers are thus consistent with its absence from inhibitory terminals in the hippocampus (Kremer et al., 2007; Wallrafen \& Dresbach, 2018). Additionally, we find low levels of Mover in the molecular layers of the DG and the stratum lacunosum-moleculare of the CA1. These are the layers that receive input from and project back to the entorhinal cortex. The fact that Mover is absent from these layers suggests that it does not influence in- or output of the hippocampus but is rather involved in intrahippocampal computation. In the inner molecular layer of the DG, Mover levels relative to Synaptophysin are slightly higher than those in the outer molecular layer. This is consistent with the typical mossy fiber projections, which not only target the apical dendrites of CA3 pyramidal neurons, but also back-project to the inner molecular layer, where they modulate themselves (Amaral et al., 2007).

In layers which are involved in intrahippocampal computation, Mover levels relative to the number of SVs are strikingly above average. This is the case in the polymorph layer (or hilus) of the DG, stratum radiatum and oriens of CA3 and CA1 and in the stratum lucidum of CA3. The polymorph layer contains some mossy fibers projections from the granule cells, which also project to the stratum lucidum, where they form giant excitatory synaptic terminals with the apical dendrites of the pyramidal neurons, which are called mossy fiber terminals. Additionally, the polymorph layer of the DG also contains terminals of the second type of DG principal cells: so-called hilar mossy cells (Scharfman et al., 2013). These project to the inner molecular layer of the ipsi- and contralateral DG. From there, they regulate basket cell activity (Sloviter, 1994). The higher Mover abundance in the inner molecular layer compared to the outer molecular layer indicates that Mover might also function in the hilar mossy cell terminals, thereby (indirectly) influencing basket cell activity and thus GABAergic signaling. Mossy fiber collaterals from the granule cells also project onto inhibitory basket cells, which in turn synapse on the granule cell somata. The projection pattern of granule mossy fiber collaterals and hilar mossy cells thus 
triggers both excitatory and inhibitory feedback onto themselves, in addition to the projection onto CA3 pyramidal neurons. High levels of Mover in these collaterals therefore suggest an involvement of Mover in modulating intrahippocampal feedback loops.

Interestingly, the distribution of Mover in excitatory mossy fiber terminals and its absence from inhibitory synapses in the stratum lucidum is reminiscent of another - evolutionarily conserved - presynaptic protein that influences mossy fiber synaptic plasticity: Tomosyn (Barak et al., 2010). Mossy fiber terminals are specialized nerve endings with a low release probability and strong capacity for facilitation (Nicoll \& Schmitz, 2005). Knockdown of Tomosyn decreases presynaptic short-term and long-term potentiation, presumably by increasing basal release probability (Ben-Simon et al., 2015). In another highly plastic synapse, the calyx of Held, Mover knockdown led to an increase in synaptic release probability (Körber et al., 2015). In the mossy fibers synapses in the stratum lucidum of CA3, however, loss of Mover only alters synaptic shortterm plasticity: while basic neuronal properties, such as release probability, are unchanged, lack of Mover results in increased frequency facilitation in an age- and calcium-dependent manner (Viotti, 2017; Viotti et al., unpublished). This change is thought to be mediated by Mover's interaction with Calmodulin, thereby influencing the cAMP-pathway (Viotti, 2017; Viotti et al., unpublished). Therefore, we assume that Mover could have a vertebrate-specific function in regulating presynaptic plasticity, which is added to the established role of the conserved protein Tomosyn.

Other layers with notably high Mover-to-Synaptophysin levels are the stratum radiatum and oriens, both of CA3 and CA1. These layers contain mainly glutamatergic axons of the hippocampal pyramidal cells. Among these are the Schaffer collaterals, that are formed by CA3 pyramidal neurons which synapse onto CA1 pyramidal neurons and associational and commissural fibers, which run within either CA3 or CA1. High Mover levels in these regions suggest that Mover may be important for intra-hippocampal information flow and processing, while in- and output are likely unaffected by Mover, due to the low expression levels in the layers associated with these processes.

\subsubsection{Mover shows a layer-specific distribution in the somatosensory system}

In our quantification study we found that Mover was enriched in layer Va of the somatosensory cortex, and virtually absent from layer IV. This distribution pattern is reminiscent of the two different input pathways to the somatosensory cortex, i.e. the lemniscal and the paralemeniscal 
paths. The differential distribution of Mover in these pathways could suggest that Mover specifically functions only in the paralemniscal pathway. In this way, Mover might indirectly influence the lemniscal input coming from the whiskers, as one proposed function of the paralemniscal path is the modulation of information transmitted via the lemniscal path (Ahissar et al., 2000; Liu et al., 2014). In this modulatory function, the paralemniscal pathway has been described to integrate input information from multiple whiskers, as compared to the whiskerspecific input from the lemniscal path. The combination and integration of both pathways is necessary to mediate precise object recognition (Liu et al., 2014). So far, the function of Mover in POm-layer Va synapses has not been described. Therefore, we can only speculate which role Mover might play in the modulation of somatosensory input. Assuming that Mover might have a "buffering"-function in this thalamocortical pathway comparable to that in the hippocampus (Viotti, 2017), Mover might dampen paralemniscal modulation of touch sensation, thereby increasing the importance of lemniscal input. Knockout of Mover might shift this balance by increasing paralemniscal modulation, thereby decreasing the somatotopy of whisker input, i.e. increasing the relative weight of the input from multiple whiskers. This in turn might influence the precision of object recognition. A combination of behavioral assays (for example whisking behavior in a gap crossing test) and electrophysiological analysis of network dynamics is needed to test these hypotheses and determine the effect of Mover in touch sensation.

Interestingly, the paralemniscal pathway has also been associated with nociception (Frangeul et al., 2014). During my work, I noticed that Mover KO animals seemed to react stronger to painful stimuli (unquantified observation). In collaboration with the lab of Dr. Manuela Schmidt (MaxPlanck-Institute for Experimental Medicine, Göttingen, Germany) we found that Mover is expressed along the nociceptive pathway, i.e. dorsal root ganglion neurons and the dorsal horn of the spinal cord, where it colocalized with markers specific for nociceptive processes (Peripherin, IB4; unpublished data). Taken together, these observations could indicate that Mover is involved in pain sensation. Behavioral tests relating to pain sensation should yield information on whether nociception is indeed altered in Mover KO animals. Mover might modulate pain sensation in a way, that nociceptive input is "buffered". Knockout of Mover might thus lead to an increased pain sensation, which would explain the observed reactions of KO mice to painful stimuli. As the KO mice also display decreased anxiety levels (Viotti et al., unpublished), stress induced analgesia might be less prominent for them, which in turn could increase pain sensitivity. 


\subsubsection{Mover levels are homogeneously high across the different} amygdaloid nuclei

While Mover is heterogeneously expressed within the hippocampus, expression levels in the different amygdaloid nuclei is homogeneously high (lateral nucleus: $\sim 40 \%$, basolateral nucleus: 47\%, medioposterior nucleus: 38\%; Wallrafen \& Dresbach, 2018). This is rather surprising, considering the diverse input and function of the different nuclei.

While Mover is mainly associated with excitatory synapses in the lateral and basolateral nuclei of the amygdala, in the medioposterior nucleus (MeP), Mover was detected at both excitatory and inhibitory synapses (Wallrafen \& Dresbach, 2018). This implies that in the MeP, Mover is also involved in GABAergic transmission. This is especially noteworthy, as many inputs related to socio-sexual behavior are processed in the MeP.

In the MeP, several types of neurons can be found. Among these are glutamatergic and GABAergic neurons. While both types can project to the hypothalamus, there is a subpopulation of GABAergic neurons that function as local interneurons and thereby modulate in- and output to and from the MeP (Keshavarzi et al., 2014). The presence of Mover at these synapses suggest that Mover might function in the processing and regulation of socio-sexual behaviors. And indeed, when knocking out Mover, mice show a phenotype related to this: mating homozygous KO mice with each other so far was unsuccessful, as females do not conceive, most likely due to a lack of interest in mating (observation by the animal care taker, personal communication). This lack of interest could be due to faulty processing of olfactory cues, such as pheromones, in the MeP, which receives its input from the main and accessory olfactory bulb (Kondo, 1992). Additionally, as the amygdala is also involved in the regulation of hormone secretion (Eleftheriou \& Zolovick, 1967), Mover KO animals might just not have enough hormone secretion to stimulate mating behavior. This hypothesis can also be corroborated by the fact that Mover - in addition to its expression in the nervous system - was found on Leydig cells in the testes of male mice (Antonini et al., 2008). Leydig cells produce testosterone under the influence of luteinizing hormone, and lack of Mover in these cells may interfere with normal testosterone production. Interestingly, the combination of one homozygous $\mathrm{KO}$ and one heterozygous animal, leads to offspring. The litters are usually smaller than in wt- or heterozygous matings, and females conceive less frequently. This in turn can also be explained by altered hormone levels, as the complex hormonal interplay before and during pregnancy is crucial for successful mating and influences litter size (Barkley \& Geschwind, 1979). 
As mentioned before, Mover expression is also high in the basolateral amygdala (BL). This nucleus has been widely connected to fear conditioning and anxiety-related behavior (Davis \& Whalen, 2001; McGarry \& Carter, 2017; McGaugh, 2004; Richardson, 1973). Mice that lack Mover indeed also show an anxiety-related phenotype: in standard behavioral tests (i.e. Morris Water maze, elevated plus maze, open field test), they move more and faster and spend more time in the open (i.e. unsheltered) parts of the maze than their WT littermates (Viotti et al., unpublished). These behaviors correspond to a reduction in anxiety levels. This reduction in anxiety might be related to the lack of Mover. As described above, Mover absence has been related to an increase in frequency facilitation in the hippocampal mossy fiber synapse (Viotti, 2017; Viotti et al., unpublished). The amygdala was described as a highly plastic structure itself, especially in the context of fear-conditioning and anxiety (Sangha \& Maren, 2015). Loss of Mover might - just like in the hippocampus - increase short term plasticity in the amygdala, resulting in the altered anxiety levels observed in the KO.

Whether the anxiety phenotype is really due to loss of Mover in the (basolateral) amygdala could be tested by generating a BL-specific, i.e. conditional, KO mouse and observing the behavior in the aforementioned tests.

Interestingly, anxiety is not the only psychological behavior Mover has been related to. In a proteome analysis of human postmortem brains, Mover was found to be significantly upregulated in the ACC in patients suffering from schizophrenia (Clark et al., 2006). Whether the upregulation in this brain region is causative of the disease, or a consequence of it, remains unknown. In the adult mouse brain, we find very low levels of Mover in the ACC ( 4.5\% below average; Wallrafen \& Dresbach, 2018). Assuming that the same is true for humans who do not suffer from schizophrenia, we can hypothesize that the upregulation of Mover in the ACC might serve as a protective mechanism: in the calyx of Held, synaptic release was dampened by knocking down Mover (Körber et al., 2015), and an upregulation of Mover in the ACC could be an attempt to confine runaway excitation. This hypothesis is corroborated by the fact that Mover expression is activity dependent, and that schizophrenia has been connected to an increase of neuronal activity in the ACC (Adam \& David, 2007).

\subsection{MOVER IN THE AUDITORY SYSTEM}

In the auditory pathway, Mover was hitherto only described at the calyx of Held, the second relay station of the binaural pathway. Knocking down Mover in the calyx changed the presynaptic release probability (Körber et al., 2015). We investigated the distribution of Mover 
in the first relay station, the VCN. There, Mover is expressed both at excitatory synapses, which arise from the auditory nerve fibers and are called endbulbs of Held, and in inhibitory synapses targeting BCs. Across these synapses, Mover again is differentially expressed, with higher absolute levels in inhibitory synapses, but higher levels relative to the number of SVs in endbulbs (Wallrafen et al., in preparation). The higher absolute values of Mover despite the lower Moverto-SV-ratios in inhibitory synapses can be explained by the fact that inhibitory synapses have more SVs than excitatory synapses.

The endbulb of Held has been subject of many studies, especially due to its strong short-term depression and thus highly plastic behavior (Yang \& Xu-Friedman, 2009). However, the exact functional purpose of this depression and its influence on information processing remain unclear. Voltage-clamp recordings show that endbulbs are rather heterogeneous in terms of the amount of depression they display. They can either be strongly depressing or not. However, sibling endbulbs - i.e. endbulbs originating from different ANF but targeting the same BCs show similar depression strengths (Yang \& Xu-Friedman, 2009). As Mover was shown to increase synaptic release probability and short-term depression, we hypothesized that the differential release probability across endbulbs corresponds to differential Mover expression levels. As a first step we therefore determined the amount of Mover per SV across all endbulbs targeting one $\mathrm{BC}$. We find that the overall input to one $\mathrm{BC}$ is homogeneous in regard to Mover levels per SV (Wallrafen et al., in preparation). As a next step, an analysis of the single endbulb terminals is necessary to determine whether the differences in depression correlate with difference in Mover expression levels. Ideally, this approach should be combined with tracing studies and functional experiments, to not only relate differences in depression behavior with difference in Mover expression, but to also determine the effect of absolute levels on endbulb synaptic physiology.

We verified the absolute lack of Mover in the KO mouse model we use (Viotti et al., unpublished) through immunofluorescence stainings and detected no fluorescence signal in either excitatory or inhibitory synapses (Wallrafen et al., in preparation).

\section{Localization of Mover and Bassoon at the AZ}

Mover was initially discovered in a yeast-2-hybrid assay using Bassoon as bait (Kremer et al., 2007). Bassoon is a vertebrate specific AZ scaffolding molecule with a molecular weight of 420kDA, which shows an extended conformation at the synaptic terminal. In its stretched conformation, it is about $80 \mathrm{~nm}$ long. The C-terminus is located around $40 \mathrm{~nm}$ from the AZ membrane (Dani et al., 2010; Gundelfinger \& Fejtova, 2012; Gundelfinger et al., 2016; Ivanova et 
al., 2015; Limbach et al., 2011; Sanmartí-Vila et al., 2000). This corresponds exactly to the diameter of one SV. While the exact binding site of Mover in the Bassoon molecule is not known, findings suggest that it is found in the C-terminal region of Bassoon. Their relative localization to each other, and the fact that Mover is associated with SVs (Ahmed et al., 2013), render it likely that Mover could function as an adapter between SVs and Bassoon. We performed colocalization analysis between Mover and Bassoon using the Pearson's correlation analysis and found surprisingly low values both in excitatory endbulbs as well as inhibitory synapses. One possible explanation for these results is the localization of the antibody epitope relative to Mover's putative binding site: the antibody used for our study was raised against amino acids 756-1001 of Bassoon, i.e. close to the N-terminus. As we are only using confocal microscopy, however, this interpretation has to be handled with care. Super-resolution microscopy, for example STED or STORM analysis, has to be employed to determine the exact localization of Mover relative to Bassoon in the presynaptic terminal of endbulbs and inhibitory synapses targeting $\mathrm{BCs}$.

\subsection{OUTLOOK}

The present study shows that Mover is heterogeneously expressed on three levels: (1) across brain areas, (2) within single brain regions and (3) between synapse types. The heterogeneous distribution lets us speculate on area- and synapse-specific functions of Mover across the mouse brain.

In the hippocampus, we find high levels of Mover in layers associated with intra-hippocampal computation. Layer-specific KO of Mover could yield information on the function of Mover at the different synapses, ultimately leading to a deeper understanding of processes such as memory formation and transition from short-term to long-term memory.

In the amygdala, Mover was highly expressed throughout the different nuclei, but differentially across the different synapse types. A detailed behavioral analysis of the $\mathrm{KO}$ mouse model is needed to determine the psycho-social effects associated with the loss of Mover.

Further studies involving nanoscopy are needed to determine the subcellular distribution of Mover in synapses targeting BCs in the VCN. STED and STORM-analysis can be applied to determine the exact binding site of Mover to the AZ-scaffolding molecule Bassoon, which remains unknown. This in turn could shed light on the interaction of the two proteins and confirm (or refute) the hypothesis that Mover functions as an adapter between SVs and the AZ in endbulbs of Held. Additionally, it could yield information on whether Mover indeed has a 
Bassoon-independent function in inhibitory synapses. Functional studies, i.e. electrophysiological measurements, could show whether the phenotype of a Mover KO can be compared to that of Bassoon disruption, i.e. whether the increase in release-probability in the Bassoon KO can be explained by downregulation of Mover expression levels. 


\section{REFERENCES}

Ackermann, F., Waites, C. L., \& Garner, C. C. (2015). Presynaptic active zones in invertebrates and vertebrates. EMBO Reports, 16(8), 923-938. http://doi.org/10.15252/embr.201540434

Adam, R., \& David, A. S. (2007). Patterns of anterior cingulate activation in schizophrenia: A selective review. Neuropsychiatric Disease and Treatment, 3(1), 87-101. http://doi.org/10.2147/nedt.2007.3.1.87

Adler, J., \& Parmryd, I. (2010). Quantifying colocalization by correlation: The pearson correlation coefficient is superior to the Mander's overlap coefficient. Cytometry Part A, 77(8), 733742.

Ahissar, E., Sosnik, R., \& Haldarilu, S. (2000). Transformation from temporal to rate coding in a somatosensory thalamocortical pathway. Nature, 406(6793), 302-306.

Ahmed, S., Wittenmayer, N., Kremer, T., Hoeber, J., Kiran Akula, A., Urlaub, H., ... Dresbach, T. (2013). Mover Is a Homomeric Phospho-Protein Present on Synaptic Vesicles. PLOS ONE, $8(5)$.

Akula, A. K. (2015). Characterization of the Functional Domains of a Novel Vertebrate Specific Presynaptic Protein - Mover. Georg-August-University Göttingen.

Alabi, A. A., \& Tsien, R. W. (2012). Synaptic Vesicle Pools and Dynamics. Cold Spring Harbor Perspectives in Biology, 4(a). http://doi.org/10.1101/cshperspect.a013680

Alibardi, L. (1998). Ultrastructural and immunocytochemical characterization of neurons in the rat ventral cochlear nucleus projecting to the inferior colliculus. Annals of Anatomy, 180(5), 415-426. http://doi.org/10.1016/S0940-9602(98)80102-7

Amaral, D. G., Scharfman, H. E., \& Lavenex, P. (2007). The dentate gyrus: fundamental neuroanatomical organization (dentate gyrus for dummies). Progress in Brain Research, 163, 3-22. http://doi.org/10.1016/S0079-6123(07)63001-5

Andersen, P., Morris, R., Amaral, D., Bliss, T., \& O'Keefe, J. (2007). The hippocampus book. New York: Oxford University Press. 
Antonini, D., Dentice, M., Mahtani, P., De Rosa, L., Gatta, G. Della, Mandinova, A., ... Missero, C. (2008). Tprg, a gene predominantly expressed in skin, is a direct target of the transcription factor p63. Journal of Investigative Dermatology, 128(7), 1676-1685.

Antonini, D., Dentice, M., Mahtani, P., Rosa, L. De, Gatta, G. Della, Mandinova, A., ... Missero, C. (2008). Tprg , a Gene Predominantly Expressed in Skin , Is a Direct Target of the Transcription Factor p63, 128. http://doi.org/10.1038/jid.2008.12

Baars, B. J., \& Gage, N. M. (2010). Cognition, Brain and Consciousness (2nd ed.). Burlington, MA 01803, USA: Elsevier Inc.

Barak, B., Williams, A., Bielopolski, N., Gottfried, I., Okun, E., Brown, M. A., ... Ashery, U. (2010). Tomosyn Expression Pattern in the Mouse Hippocampus Suggests Both Presynaptic and Postsynaptic Functions. Frontiers in Neuroanatomy, 4(12), 1-11.

Barkley, M. S., \& Geschwind, I. I. (1979). The Gestational Pattern of Estradiol, Testosterone and Progesterone Secretion in Selected Strains of Mice. Biology of Reproduction, 20, 733-738.

Basu, J., Betz, A., Brose, N., \& Rosenmund, C. (2007). Munc13-1 C1 Domain Activation Lowers the Energy Barrier for Synaptic Vesicle Fusion. Journal of Neuroscience, 27(5), 1200-1210. http://doi.org/10.1523/JNEUROSCI.4908-06.2007

Ben-Simon, Y., Rodenas-Ruano, A., Alviña, K., Lam, A. D., Stuenkel, E. L., Castillo, P. E., \& Ashery, U. (2015). A Combined Optogenetic-Knockdown Strategy Reveals a Major Role of Tomosyn in Mossy Fiber Synaptic Plasticity. Cell Reports, 12(3), 396-404.

Bennett, M., Calakos, N., \& Scheller, R. (1992). Syntaxin: a synaptic protein implicated in docking of synaptic vesicles at presynaptic active zones. Science, 257(5067), 255-9.

Blackman, A. V., Abrahamsson, T., Costa, R. P., Lalanne, T., \& Sjöström, P. J. (2013). Target-cellspecific short-term plasticity in local circuits. Frontiers in Synaptic Neuroscience, 5(12), $1-$ 13.

Borst, J. G. G., \& Soria van Hoeve, J. (2012). The Calyx of Held Synapse: From Model Synapse to Auditory Relay. Annual Review of Physiology, 74(1), 199-224. http://doi.org/10.1146/annurev-physiol-020911-153236

Bosman, L. W. J., Houweling, A. R., Owens, C. B., Tanke, N., Shevchouk, O. T., Rahmati, N., ... De Zeeuw, C. I. (2011). Anatomical Pathways Involved in Generating and Sensing Rhythmic 
Whisker Movements. Frontiers in Integrative Neuroscience, 5(12), 1-28.

Brodal, P. (2010). The Central Nervous System: Structure and Function. New York: Oxford University Press.

Bucurenciu, I., Kulik, A., Schwaller, B., Frotscher, M., \& Jonas, P. (2008). Nanodomain Coupling between $\mathrm{Ca} 2+$ Channels and Ca2+Sensors Promotes Fast and Efficient Transmitter Release at a Cortical GABAergic Synapse. Neuron, 57(4), 536-545. http://doi.org/10.1016/j.neuron.2007.12.026

Burré, J., Beckhaus, T., Corvey, C., Karas, M., Zimmermann, H., \& Volknandt, W. (2006). Synaptic vesicle proteins under conditions of rest and activation: Analysis by 2-D difference gel electrophoresis. Electrophoresis, 27(17), 3488-3496.

Cai, Q., Pan, P.-Y., \& Sheng, Z.-H. (2007). Syntabulin-Kinesin-1 Family Member 5B-Mediated Axonal Transport Contributes to Activity-Dependent Presynaptic Assembly. Journal of Neuroscience, 27(27), 7284-7296.

Castejon, C., Barros-Zulaica, N., \& Nunez, A. (2016). Control of somatosensory cortical processing by thalamic posterior medial nucleus: A new role of thalamus in cortical function. PLOS ONE, 11(1), 1-33. http://doi.org/10.1371/journal.pone.0148169

Chad, J. E., \& Eckert, R. (1984). Calcium domains associated with individual channels can account for anomalous voltage relations of Ca-dependent responses. Biophysical Journal, 45(May), 993-999.

Charette, S. J., Lambert, H., Nadeau, P. J., \& Landry, J. (2010). Protein quanti fi cation by chemiluminescent Western blotting : Elimination of the antibody factor by dilution series and calibration curve. Journal of Immunological Methods, 353(1-2), 148-150. http://doi.org/10.1016/j.jim.2009.12.007

Chaudhry, F. a., Reimer, R. J., Bellocchio, E. E., Danbolt, N. C., Osen, K. K., Edwards, R. H., \& Storm-Mathisen, J. (1998). The vesicular GABA transporter, VGAT, localizes to synaptic vesicles in sets of glycinergic as well as GABAergic neurons. Journal of Neuroscience, 18(23), 9733-9750.

Clark, D., Dedova, I., Cordwell, S., \& Matsumoto, I. (2006). A proteome analysis of the anterior cingulate cortex gray matter in schizophrenia. Molecular Psychiatry, 11, 459-470. 
Dani, A., Huang, B., Bergan, J., Dulac, C., \& Zhuang, X. (2010). Superresolution Imaging of Chemical Synapses in the Brain. Neuron, 68(5), 843-856. http://doi.org/10.1016/j.neuron.2010.11.021

Davis, M., \& Whalen, P. J. (2001). The amygdala: Vigilance and emotion. Molecular Psychiatry, $6(1), 13-34$.

Dobrunz, L. E., \& Stevens, C. F. (1997). Heterogeneity of release probability, facilitation, and depletion at central synapses. Neuron, 18(6), 995-1008. http://doi.org/10.1016/S08966273(00)80338-4

Dolphin, A. C. (2009). Calcium channel diversity: multiple roles of calcium channel subunits. Current Opinion in Neurobiology, 19(3), 237-244. http://doi.org/10.1016/j.conb.2009.06.006

Dresbach, T., Torres, V., Wittenmayer, N., Altrock, W. D., Zamorano, P., Zuschratter, W., ... Gundelfinger, E. D. (2006). Assembly of active zone precursor vesicles: Obligatory trafficking of presynaptic cytomatrix proteins Bassoon and Piccolo via A trans-Golgi compartment. Journal of Biological Chemistry, 281(9), 6038-6047. http://doi.org/10.1074/jbc.M508784200

Dunn, K. W., Kamocka, M. M., \& McDonald, J. H. (2011). A practical guide to evaluating colocalization in biological microscopy. Am J Physiol Cell Physiol, 300, C723-C742.

Eleftheriou, B. E., \& Zolovick, A. J. (1967). Effect of amygdaloid lesions on plasma and pituitary levels of luteinizing hormone. J. Reprod. Fert., 14, 33-37.

Elliott, R., Zahn, R., Deakin, J. F. W., \& Anderson, I. M. (2011). Affective cognition and its disruption in mood disorders. Neuropsychopharmacology, 36(1), 153-182. http://doi.org/10.1038/npp.2010.77

Emes, R. D., Pocklington, A. J., Anderson, C. N. G., \& Bayes, A. (2008). Evolutionary expansion and anatomical specialization of synapse proteome complexity. Nat Neuroscience, 11(7), 799-806.

Fejtova, A., \& Gundelfinger, E. (2006). Molecular Organization and Assembly of the Presynaptic Active Zone of Neurotransmitter Release. In Cell Communications in Nervous and Immune System. Results and Problems in Cell Differentiation. Springer, Berlin, Heidelberg. 
Fernandez-Fewell, G. D., \& Meredith, M. (1994). C-Fos Expression in Vomeronasal Pathways of Mated or Pheromone-Stimulated Male Golden Hamsters: Contributions From Vomeronasal Sensory Input and Expression Related To Mating Performance. Journal of Neuroscience, 14(6), 3643-3654.

Förster, E., Zhao, S., \& Frotscher, M. (2006). Laminating the hippocampus. Nature Reviews Neuroscience, 7(4), 259-267.

Frangeul, L., Porrero, C., Garcia-Amado, M., Maimone, B., Maniglier, M., Clascá, F., \& Jabaudon, D. (2014). Specific activation of the paralemniscal pathway during nociception. European Journal of Neuroscience, 39(9), 1455-1464.

George, J. M. (2002). The synucleins. Genome Biology, 3(1), 1-6.

Ghelani, T. (2016). Orientation and organization of the presynaptic active zone protein Bassoon : from the Golgi to the synapse. Georg-August-University Göttingen.

Goedert, M. (2001). Alpha-Synuclein and neurodegenerative diseases. Nature Reviews, 2(July), 492-501.

Groffen, A. J., Martens, S., Arazola, R. D., Cornelisse, L. N., Lozovaya, N., Jong, A. P. H. De, ... Verhage, M. (2010). Doc2b is a high-affinity Ca2+ sensory for spontaneous neurotransmitter release. Science, 327(May), 1614-1618.

Gundelfinger, E. D., \& Fejtova, A. (2012). Molecular organization and plasticity of the cytomatrix at the active zone. Current Opinion in Neurobiology, 22(3), 423-430. http://doi.org/10.1016/j.conb.2011.10.005

Gundelfinger, E. D., Reissner, C., \& Garner, C. C. (2016). Role of Bassoon and Piccolo in Assembly and Molecular Organization of the Active Zone. Frontiers in Synaptic Neuroscience, 7(1).

Hallermann, S., Fejtova, A., Schmidt, H., Weyhersmüller, A., Silver, R. A., Gundelfinger, E. D., \& Eilers, J. (2010). Bassoon speeds vesicle reloading at a central excitatory synapse. Neuron, 68(4), 710-723.

Hallermann, S., Pawlu, C., Jonas, P., \& Heckmann, M. (2003). A large pool of releasable vesicles in a cortical glutamatergic synapse. Proceedings of the National Academy of Sciences, 100(15), 8975-8980. http://doi.org/10.1073/pnas.1432836100 
Heidebrecht, F., Heidebrecht, A., Schulz, I., Behrens, S., \& Bader, A. (2009). Improved semiquantitative Western blot technique with increased quanti fi cation range. Journal of Immunological Methods, 345(1-2), 40-48. http://doi.org/10.1016/j.jim.2009.03.018

Hsu, F. S., Hu, F., \& Mao, Y. (2015). Spatiotemporal control of phosphatidylinositol 4-phosphate by Sac2 regulates endocytic recycling. Journal of Cell Biology, 209(1), 97-110. http://doi.org/10.1083/jcb.201408027

Imig, C., Min, S. W., Krinner, S., Arancillo, M., Rosenmund, C., Südhof, T. C., ... Cooper, B. H. (2014). The Morphological and Molecular Nature of Synaptic Vesicle Priming at Presynaptic Active Zones. Neuron, 84(2), 416-431. http://doi.org/10.1016/j.neuron.2014.10.009

Ishiyama, S., Schmidt, H., Cooper, B. H., Brose, N., \& Eilers, J. (2014). Munc13-3 Superprimes Synaptic Vesicles at Granule Cell-to-Basket Cell Synapses in the Mouse Cerebellum. Journal of Neuroscience, 34(44), 14687-14696. http://doi.org/10.1523/JNEUROSCI.2060-14.2014

Ivanova, D., Dirks, A., Montenegro-venegas, C., Schöne, C., Altrock, W. D., Marini, C., ... Fejtova, A. (2015). Synaptic activity controls localization and function of CtBP 1 via binding to Bassoon and Piccolo, 34(8).

Jahn, R., \& Südhof, T. C. (1994). Synaptic Vesicles and Exocytosis. Annual Review of Neuroscience, 17, 219-46.

Jean, P., de la Morena, D. L., Michanski, S., Tobón, L. M. J., Chakrabarti, R., Picher, M. M., ... Moser, T. (2018). The synaptic ribbon is critical for sound encoding at high rates and with temporal precision. ELife, 7, 1-39. http://doi.org/10.7554/eLife.29275

Jung, S., Oshima-Takago, T., Chakrabarti, R., Wong, A. B., Jing, Z., Yamanbaeva, G., ... Moser, T. (2015). Rab3-interacting molecules $2 \alpha$ and $2 \beta$ promote the abundance of voltage-gated $\mathrm{Ca}$ $\vee 1.3 \mathrm{Ca}^{2+}$ channels at hair cell active zones. Proceedings of the National Academy of Sciences, 112(24), E3141-E3149. http://doi.org/10.1073/pnas.1417207112

Kaeser, P. S., Deng, L., Wang, Y., Dulubova, I., Liu, X., Rizo, J., \& Südhof, T. C. (2011). RIM proteins tether Ca2+channels to presynaptic active zones via a direct PDZ-domain interaction. Cell, 144(2), 282-295. http://doi.org/10.1016/j.cell.2010.12.029

Karlsson, U., \& Schultz, R. (1965). Fixation of the Central Nervous System from Electron Microscopy by Aldehyde Perfusion. I. Preservation with Aldehyde Perfusates versus direct 
Perfusion with Osmium Tetroxide with special Reference to Membranes and the Extracellular Space. Journal of Ultrastructure Research, Feb(12), 160-86.

Keshavarzi, S., Sullivan, R. K. P., lanno, D. J., \& Sah, P. (2014). Functional Properties and Projections of Neurons in the Medial Amygdala. Journal of Neuroscience, 34(26), 86998715.

Kondo, Y. (1992). Lesions of the medial amygdala produce severe impairment of copulatory behavior in sexually inexperienced male rats. Physiology\&Behavior, 51(5), 939-943.

Körber, C., Horstmann, H., Venkataramani, V., Herrmannsdörfer, F., Kremer, T., Kaiser, M., ... Kuner, T. (2015). Modulation of Presynaptic Release Probability by the Vertebrate-Specific Protein Mover. Neuron, 87(3), 521-533.

Körber, C., \& Kuner, T. (2016). Molecular machines regulating the release probability of synaptic vesicles at the active zone. Frontiers in Synaptic Neuroscience, 8(MAR), 1-17. http://doi.org/10.3389/fnsyn.2016.00005

Kremer, J. R., Mastronarde, D. N., \& McIntosh, J. R. (1996). Computer visualization of threedimensional image data using IMOD. Journal of Structural Biology, 116(1), 71-76. http://doi.org/10.1006/jsbi.1996.0013

Kremer, T. (2008). Identification and characterisation of mover as a novel vertebrate-specific presynaptic protein. Ruperto-Carola University Heidelberg.

Kremer, T., Kempf, C., Wittenmayer, N., Nawrotzki, R., Kuner, T., Kirsch, J., \& Dresbach, T. (2007). Mover is a novel vertebrate-specific presynaptic protein with differential distribution at subsets of CNS synapses. FEBS Letters, 581(24), 4727-4733.

LeDoux, J. E., Cicchetti, P., Xagoraris, A., \& Romanski, L. M. (1990). The Lateral Amygdaloid in Fear Conditioning Nucleus : Sensory Interface Amygdala. Journal of Neuroscience, 10(4), 1062-1069.

Lee, J. S., Ho, W.-K., Neher, E., \& Lee, S.-H. (2013). Superpriming of synaptic vesicles after their recruitment to the readily releasable pool. Proceedings of the National Academy of Sciences, 110(37), 15079-15084. http://doi.org/10.1073/pnas.1314427110

Limbach, C., Laue, M. M., Wang, X., Hu, B., Thiede, N., Hultqvist, G., \& Kilimann, M. W. (2011). Molecular in situ topology of Aczonin/Piccolo and associated proteins at the mammalian 
neurotransmitter release site. Proceedings of the National Academy of Sciences, 108(31), E392-E401. http://doi.org/10.1073/pnas.1101707108

Liu, J., Liu, B., Zhang, X., Yu, B., Guan, W., Wang, K., ... Zhao, C. (2014). Calretinin-positive L5a pyramidal neurons in the development of the paralemniscal pathway in the barrel cortex. Mol Brain, 7(1), 84. http://doi.org/10.1186/s13041-014-0084-8

Ma, C., Su, L., Seven, A. B., Xu, Y., \& Rizo, J. (2012). Reconstitution of the Vital Functions of Munc18 and Munc13 in Neurotransmitter Release. Science, (December). Retrieved from http://science.sciencemag.org/

Mahan, A. L., \& Ressler, K. J. (2012). Fear conditioning, synaptic plasticity, and the amygdala: implications for posttraumatic stress disorder. Trends Neurosci., 35(1), 24-35.

Mastronarde, D. N. (2005). Automated electron microscope tomography using robust prediction of specimen movements. Journal of Structural Biology, 152(1), 36-51. http://doi.org/10.1016/j.jsb.2005.07.007

McDonald, A. J., Mascagni, F., \& Guo, L. (1996). Projections of the medial and lateral prefrontal cortices to the amygdala: A Phaseolus vulgaris leucoagglutinin study in the rat. Neuroscience, $71(1), 55-75$.

McGarry, L. M., \& Carter, A. G. (2017). Prefrontal Cortex Drives Distinct Projection Neurons in the Basolateral Amygdala. Cell Reports, 21(6), 1426-1433.

McGaugh, J. L. (2004). the Amygdala Modulates the Consolidation of Memories of Emotionally Arousing Experiences. Annual Review of Neuroscience, 27(1), 1-28. http://doi.org/10.1146/annurev.neuro.27.070203.144157

Mendoza Schulz, A., Jing, Z., María Sánchez Caro, J., Wetzel, F., Dresbach, T., Strenzke, N., ... Moser, T. (2014). Bassoon-disruption slows vesicle replenishment and induces homeostatic plasticity at a CNS synapse. EMBO Journal, 33(5), 512-527.

Mezey, E., Dehejia, A., Harta, G., Papp, M. I., Polymeropoulos, M. H., \& Brownstein, M. J. (1998). Alpha synuclein in neurodegenerative disorders: murder or accomplice? Nature Medicine, 4(7), 755-757.

Michelassi, F., Liu, H., Hu, Z., \& Dittman, J. S. (2017). A C1-C2 Module in Munc13 Inhibits Calcium-Dependent Neurotransmitter Release. Neuron, 95(3), 577-590.e5. 
http://doi.org/10.1016/j.neuron.2017.07.015

Micheva, K. D., Busse, B., Weiler, N. C., O’Rourke, N., \& Smith, S. J. (2010). Single-synapse analysis of a diverse synapse population: Proteomic imaging methods and markers. Neuron, 68(4), 639-653.

Mukherjee, K., Yang, X., Gerber, S. H., Kwon, H.-B., Ho, A., Castillo, P. E., ... Sudhof, T. C. (2010). Piccolo and bassoon maintain synaptic vesicle clustering without directly participating in vesicle exocytosis. Proceedings of the National Academy of Sciences, 107(14), 6504-6509. http://doi.org/10.1073/pnas.1002307107

Mural, R. J., Adams, M. D., Myers, E. W., Smith, H. O., Gabor Miklos, G. L., Wides, R., ... Craig Venter, J. (2002). A comparison of whole-genome shotgun-derived mouse chromosome 16 and the human genome. Science, 296(5573), 1661-1671. http://doi.org/10.1126/science.1069193

Navone, F., Jahn, R., Gioia, G. Di, Stukenbrok, H., Greengard, P., \& Camilli, P. De. (1986). Protein p38: An integral membrane protein specific for small vesicles of neurons and neuroendocrine cells. Journal of Cell Biology, 103(6), 2511-2527.

Neher, E. (1998). Vesicle Pools and Ca2+ Microdomains: New Tools for Understanding Their Roles in Neurotransmitter Release. Neuron, 20(March), 389-399. http://doi.org/10.1063/1.4980717

Nicolelis, M. A. L. (2005). Computing with thalamocortical ensembles during different behavioural states. Journal of Physiology, 566(1), 37-47.

Nicoll, R. A., \& Schmitz, D. (2005). Synaptic plasticity at hippocampal mossy fibre synapses. Nature Reviews Neuroscience, 6(11), 863-876.

O'Keefe, J., \& Nadel, L. (1978). The Hippocampus as a Cognitive Map. New York: Oxford University Press.

Okerlund, N. D., Schneider, K., Leal-Ortiz, S., Montenegro-Venegas, C., Kim, S. A., Garner, L. C., ... Garner, C. C. (2017). Bassoon Controls Presynaptic Autophagy through Atg5. Neuron, 93(4), 897-913.e7.

Oyler, G. A., Higgins, G. A., Hart, R. A., Battenberg, E., Billingsley, M., Bloom, F. E., \& Wilson, M. C. (1989). The identification of a novel synaptosomal-associated protein, SNAP-25, 
differentially expressed by neuronal subpopulations. Journal of Cell Biology, 109(6 I), 30393052. http://doi.org/10.1083/jcb.109.6.3039

Pang, Z. P., Bacaj, T., Yang, X., Zhou, P., Xu, W., \& Su, T. C. (2011). Doc2 Supports Spontaneous Synaptic Transmission by a Ca 2 + -Independent Mechanism. http://doi.org/10.1016/j.neuron.2011.03.011

Paxinos, G., \& Franklin, K. B. J. (2001). The Mouse Brain in Stereotaxic Coordinates (2nd editio). San Diego: ACADEMIC PRESS.

Petersen, C. C. H. (2007). The functional organization of the barrel cortex. Neuron, 56(2), 339355. http://doi.org/10.1016/j.neuron.2007.09.017

Qu, L., Akbergenova, Y., Hu, Y., \& Schikorski, T. (2009). Synapse-to-synapse variation in mean synaptic vesicle size and its relationship with synaptic morphology and function. Journal of Comparative Neurology, 514(4), 343-352. http://doi.org/10.1002/cne.22007

Richardson, J. (1973). The amygdala: historical and functional analysis. Acta Neurobiol Exp, 33(3), 623-648.

Rizzoli, S. O., \& Betz, W. J. (2005). Synaptic vesicle pools. Nature Reviews Neuroscience, 6(1), 5769. http://doi.org/10.1038/nrn1583

Rollenhagen, A., \& Lübke, J. H. R. (2010). The mossy fiber bouton: The "common" or the "unique" synapse? Frontiers in Synaptic Neuroscience, 2(MAR), 1-9. http://doi.org/10.3389/fnsyn.2010.00002

Rollenhagen, A., Satzler, K., Rodriguez, E. P., Jonas, P., Frotscher, M., \& Lubke, J. H. R. (2007). Structural Determinants of Transmission at Large Hippocampal Mossy Fiber Synapses. Journal of Neuroscience, 27(39), 10434-10444. http://doi.org/10.1523/JNEUROSCI.194607.2007

Rosenmund, C., \& Stevens, C. F. (1996). Definition of the readily releasable pool of vesicles at hippocampal synapses. Neuron, 16(6), 1197-1207. http://doi.org/10.1016/S08966273(00)80146-4

Ryan, T. J., \& Grant, S. G. N. (2009). The origin and evolution of synapses. Nature Reviews Neuroscience, 10(11), 701-712. 
Sangha, S., \& Maren, S. (2015). Plasticity of Fear and Safety Neurons of the Amygdala in Response to Fear Extinction. Frontiers in Behavioral Neuroscience, 9(12), 1-10.

Sanmartí-Vila, L., Tom Dieck, S., Richter, K., Altrock, W., Zhang, L., Volknandt, W., ... Dresbach, T. (2000). Membrane association of presynaptic cytomatrix protein Bassoon. Biochemical and Biophysical Research Communications, 275(1), 43-46. http://doi.org/10.1006/bbrc.2000.3256

Scharfman, H. E., Myers, C. E., Frotscher, M., \& Ludwigs, A. (2013). Hilar mossy cells of the dentate gyrus : a historical perspective, 6(January), 1-17. http://doi.org/10.3389/fncir.2012.00106

Schlüter, O. M., Basu, J., Südhof, T. C., \& Rosenmund, C. (2006). Rab3 Superprimes Synaptic Vesicles for Release: Implications for Short-Term Synaptic Plasticity. Journal of Neuroscience, 26(4), 1239-1246. http://doi.org/10.1523/JNEUROSCI.3553-05.2006

Schneggenburger, R., Sakaba, T., \& Neher, E. (2002). Vesicle pools and short-term synaptic depression: Lessons from a large synapse. Trends in Neurosciences, 25(4), 206-212. http://doi.org/10.1016/S0166-2236(02)02139-2

Schubert, D., Kötter, R., \& Staiger, J. F. (2007). Mapping functional connectivity in barrel-related columns reveals layer- and cell type-specific microcircuits. Brain Structure and Function, 212(2), 107-119.

Shlosberg, D., Amitai, Y., \& Azouz, R. (2006). Time-Dependent, Layer-Specific Modulation of Sensory Responses Mediated by Neocortical Layer 1. Journal of Neurophysiology, 96(6), 3170-3182. http://doi.org/10.1152/jn.00520.2006

Simon, S. M., \& Llinás, R. R. (1985). Compartmentalization of the submembrane calcium activity during calcium influx and its significance in transmitter release. Biophysical Journal, 48(3), 485-498. http://doi.org/10.1016/S0006-3495(85)83804-2

Sloviter, R. S. (1994). The Functional Organization of the Hippocampal Dentate Gyms and Its Relevance to the Pathogenesis of Temporal Lobe Epilepsy.

Südhof, T. C. (2012). The presynaptic active zone. Neuron, 75(1), 11-25.

Suneja, S. K., Benson, C. G., Gross, J., \& Potashner, S. J. (1995). Evidence for Glutamatergic Projections from the Cochlear Nucleus to the Superior Olive and the Ventral Nucleus of the 
Lateral Lemniscus. Journal of Neurochemistry, 64(1), 161-171.

http://doi.org/10.1046/j.1471-4159.1995.64010161.x

Takao-Rikitsu, E., Mochida, S., Inoue, E., Deguchi-Tawarada, M., Inoue, M., Ohtsuka, T., \& Takai, Y. (2004). Physical and functional interaction of the active zone proteins, CAST, RIM1, and Bassoon, in neurotransmitter release. Journal of Cell Biology, 164(2), 301-311. http://doi.org/10.1083/jcb.200307101

Tarsa, L., \& Goda, Y. (2002). Synaptophysin regulates activity-dependent synapse formation in cultured hippocampal neurons. Proceedings of the National Academy of Sciences, 99(2), 1012-1016. http://doi.org/10.1073/pnas.022575999

Taschenberger, H., Woehler, A., \& Neher, E. (2016). Superpriming of synaptic vesicles as a common basis for intersynapse variability and modulation of synaptic strength. Proceedings of the National Academy of Sciences, 113(31), E4548-E4557. http://doi.org/10.1073/pnas.1606383113

Taylor, S. C., Berkelman, T., Yadav, G., \& Hammond, M. (2013). A Defined Methodology for Reliable Quantification of Western Blot Data. http://doi.org/10.1007/s12033-013-9672-6

Toki, M. I., Cecchi, F., Hembrough, T., Syrigos, K. N., \& Rimm, D. L. (2017). Proof of the quantitative potential of immunofluorescence by mass spectrometry. Laboratory Investigation, 97(3), 329-334. http://doi.org/10.1038/labinvest.2016.148

tom Dieck, S., Altrock, W. D., Kessels, M. M., Qualmann, B., Regus, H., Brauner, D., ... Brandstätter, J. H. (2005). Molecular dissection of the photoreceptor ribbon synapse: Physical interaction of Bassoon and RIBEYE is essential for the assembly of the ribbon complex. Journal of Cell Biology, 168(5), 825-836. http://doi.org/10.1083/jcb.200408157

tom Dieck, S., Sanmartí-Vila, L., Langnaese, K., Richter, K., Kindler, S., Soyke, A., ... Gundelfinger, E. D. (1998). Bassoon, a novel zinc-finger CAG/glutamine-repeat protein selectively localized at the active zone of presynaptic nerve terminals. Journal of Cell Biology, 142(2), 499-509. http://doi.org/PMC2133055

Trimble, W. S., Cowan, D. M., \& Scheller, R. H. (1988). VAMP-1: A synaptic vesicle-associated integral membrane protein (neuron-specific protein/transmembrane protein/proline-rich domain/Torpedo electric organ/synapsin I). Neurobiology, 85(June), 4538-4542. Retrieved from https://www.ncbi.nlm.nih.gov/pmc/articles/PMC280466/pdf/pnas00264-0432.pdf 
Trinh, K., \& Storm, D. R. (2003). Vomeronasal organ detects odorants in absence of signaling through main olfactory epithelium. Nature Neuroscience, 6(5), 519-525. http://doi.org/10.1038/nn1039

Tsuriel, S., Geva, R., Zamorano, P., Dresbach, T., Boeckers, T., Gundelfinger, E. D., ... Ziv, N. E. (2006). Local sharing as a predominant determinant of synaptic matrix molecular dynamics. PLoS Biology, 4(9), 1572-1587. http://doi.org/10.1371/journal.pbio.0040271

Varoqueaux, F., Sigler, A., Rhee, J.-S., Brose, N., Enk, C., Reim, K., \& Rosenmund, C. (2002). Total arrest of spontaneous and evoked synaptic transmission but normal synaptogenesis in the absence of Munc13-mediated vesicle priming. Proceedings of the National Academy of Sciences, 99(13), 9037-9042. http://doi.org/10.1073/pnas.122623799

Verhage, M., Maia, A. S., Plomp, J. J., Brussaard, A. B., Heeroma, J. H., Vermeer, H., ... Südhof, T. C. (2000). Synaptic Assembly of the Brain in the Absence of Neurotransmitter Secretion. Science, (February).

Viotti, J. S. (2017). The presynaptic protein Mover buffers synaptic plasticity at the hippocampal mossy fiber synapse. Georg-August-University Göttingen.

Viotti, J. S., Ott, F. W., Schleicher, E. M., Wagner, J., Bouter, Y., Bayer, T. A., \& Dresbach, T. (n.d.). Increased synaptic facilitation and exploratory behavior in mice lacking the presynaptic protein Mover. Submitted.

Von Gersdorff, H., Vardi, E., Matthews, G., \& Sterling, P. (1996). Evidence that vesicles on the synaptic ribbon of retinal bipolar neurons can be rapidly released. Neuron, 16(6), 12211227. http://doi.org/10.1016/S0896-6273(00)80148-8

Waites, C. L., Leal-Ortiz, S. A., Okerlund, N., Dalke, H., Fejtova, A., Altrock, W. D., ... Garner, C. C. (2013). Bassoon and Piccolo maintain synapse integrity by regulating protein ubiquitination and degradation. EMBO Journal, 32(7), 954-969.

Wallrafen, R., \& Dresbach, T. (2018). The Presynaptic Protein Mover Is Differentially Expressed Across Brain Areas and Synapse Types. Frontiers in Neuroanatomy, 12, 58. http://doi.org/10.3389/fnana.2018.00058

Wallrafen, R., Dresbach, T., \& Viotti, J. S. (n.d.). Quantifying the Heterogeneous Distribution of a Synaptic Protein in the Mouse Brain Using Immunofluorescence. Journal of Visualized 


\section{Experiments.}

Wallrafen, R., Hintze, A., Wetzel, F., Wichmann, C., \& Dresbach, T. (n.d.). Mover has differential effects on Synaptic-vesicle-to-active-zone-distance at Endbulbs of Held and inhibitory synapses targeting bushy cells in the VCN. In Preparation.

Wang, X., Hu, B., Zieba, A., Neumann, N. G., Kasper-Sonnenberg, M., Honsbein, A., ... Kilimann, M. W. (2009). A Protein Interaction Node at the Neurotransmitter Release Site: Domains of Aczonin/Piccolo, Bassoon, CAST, and Rim Converge on the N-Terminal Domain of Munc131. Journal of Neuroscience, 29(40), 12584-12596. http://doi.org/10.1523/JNEUROSCI.125509.2009

Waters, J., \& Smith, S. J. (2002). Vesicle pool partitioning influences presynaptic diversity and weighting in rat hippocampal synapses. Journal of Physiology, 541(3), 811-823. http://doi.org/10.1113/jphysiol.2001.013485

Welker, C. (1971). Microelectrode delineation of fine grain somatotopic organization of (Sml) cerebral neocortex in albino rat. Brain Res., 26(2), 259-75.

Welker, C., \& Woolsey, T. A. (1974). Structure of layer IV in the somatosensory neocortex of the rat: Description and comparison with the mouse. Journal of Comparative Neurology, 158(4), 437-453.

Wilhelm, B. G., Mandad, S., Truckenbrodt, S., Kröhnert, K., Schäfer, C., Rammner, B., ... Rizzoli, S. O. (2014). Composition of isolated synaptic boutons reveals the amounts of vesicle trafficking proteins. Science, 344(6187), 1023-1028. http://doi.org/10.1126/science.1252884

Wong, A. B., Rutherford, M. A., Gabrielaitis, M., Pangršič, T., Göttfert, F., Frank, T., ... Moser, T. (2014). Developmental refinement of hair cell synapses tightens the coupling of Ca2+influx to exocytosis. EMBO Journal, 33(3), 247-264. http://doi.org/10.1002/embj.201387110

Yang, H., \& Xu-Friedman, M. A. (2009). Impact of Synaptic Depression on Spike Timing at the Endbulb of Held. Journal of Neurophysiology, 102(3), 1699-1710. http://doi.org/10.1152/jn.00072.2009

Young, E. D., \& Oertel, D. (2003). Cochlear nucleus. In G. M. Shepherd (Ed.), The synaptic organization of the brain (pp. 125-163). New York: Oxford University Press. 
Young, E. D., \& Oertel, D. (2010). Cochlear nucleus. In G. M. Shepherd \& S. Grillner (Eds.), Handbook of brain microcircuits (pp. 215-223). New York: Oxford University Press.

Yu, C., Derdikman, D., Haidarliu, S., \& Ahissar, E. (2006). Parallel thalamic pathways for whisking and touch signals in the rat. PLoS Biology, 4(5), 819-825.

Ziegler, D. R., Cullinan, W. E., \& Herman, J. P. (2002). Distribution of vesicular glutamate transporter mRNA in rat hypothalamus. Journal of Comparative Neurology, 448(3), 217229. 


\section{ACKNOWLEDGEMENTS}

First and foremost, I would like to thank my supervisor, Thomas Dresbach, for his constant support during my Master's thesis and my PhD time. In addition to guiding me through this time, he always displayed a level of friendship that is more than any PhD student can hope for! I would also like to thank my thesis advisory committee: Prof. Nils Brose, Prof. Paul Lingor and Dr. Wiebke Möbius, for fruitful scientific discussions during the committee meetings.

Additionally, Prof. Jochen Staiger and his lab supported me throughout this time, both with technical expertise as well as providing me with the possibility to use their cryostat.

My gratitude also goes to Dr. Camin Dean and Dr. Nils Halbsgut (now working at Carl Zeiss) from the European Neuroscience Institute, for support and the possibility to use their confocal microscope.

Without my labmates, my PhD time would not have been nearly as fun as it was.Thank you, Andoniya, Tina, Julio, Hermes, Donatus, Moritz and Jeremy. Special thanks goes to Irmgard, the best TA anyone can hope for. Julio and Andoniya (surely with support from Nevi ;), my thanks to you for your comments on this thesis. I would also like to acknowledge my two brilliant lab rotation students Klara and Hendrik, who let me practice my teaching skills on them :)

Robin, who is without a doubt the SAP-consultant with the biggest Neuroscience-related knowledge, for being there, for better and for worse, and for helping me change perspectives once in a while. Laurin and Moritz, who helped me take a step back and see the world through a child's eyes (again).

My family and friends, especially my mother, without who my sister and I would not be the women we are today.

Everyone who supported me during and with this thesis, especially Alex, Anouk and Marilen for valuable comments.

And last - but definitely not least - I would like to offer my deepest gratitude to all the experimental animals who had to give their lives so that we can - in the long run - use the knowledge gained through their sacrifice to improve the lives of others. 


\section{LIST OF ABBREVIATIONS}

ACC

Anterior cingulate cortex

ANF

Auditory nerve fiber

$A Z$

Active zone

$\mathrm{BC}$

Bushy cells

$\mathrm{BL}$

Basolateral nucleus of the amygdala

CA

Cornu Ammonis

CAZ

Cytomatrix of the active zone

DG

Dentate gyrus

GABA

$\gamma$-amino butyric acid

KO

Mover knockout mice

MeP

Medioposterior nucleus of the amygdala

POm

Posterior medial nucleus of the thalamus

RIM

Rab-interacting molecule

$\mathrm{RP}$

Resting pool

RRP

Readily-releasable pool

S1

Somatosensory cortex

SNARE

Soluble $\mathrm{N}$-ethylmaleimide sensitive factor attachment protein receptor

SV

Synaptic vesicle

VCN

Ventral cochlear nucleus

$\mathrm{VPm}$

Ventroposterior nucleus of the thalamus

WT

Wild type mice 


\section{LIST OF FIGURES}

\section{Introduction}

Figure 1: The somatosensory system in mice. 11

Figure 2: Schematic overview on the auditory system of mice. 13

Figure 3: Drawing of the hippocampus by Santiago Ramón y Cajal. 14

Figure 4: Schematic overview of the input to the amygdala. 16

\section{Chapter 2}

Figure 1: Immunofluorescence images of DAPI, mover and synaptophysin at the 5 rostro-caudal levels. 34

Figure 2: Quantification of the mover distribution across the 5 rostro-caudal levels...........36

Figure 3: Mover distribution in the mouse hippocampus. 37

\section{Chapter 3}

Figure 1: Immunofluorescence images of DAPI, mover and synaptophysin at the 5 rostro-caudal levels. 48

Figure 2: Quantification of the mover distribution across the 5 rostro-caudal levels. .50

Figure 3: Mover distribution in the mouse hippocampus. .52

Figure 4: Mover colocalization with presynaptic markers vGlut1 and vGAT in the mouse hippocampus. 54

Figure 5: Mover distribution in the mouse amygdala . .56

Figure 6: Mover colocalization with presynaptic markers vGlut1 and vGAT in the mouse amygdala. 58

\section{Chapter 4}

Figure 1: Mover is present in the ventral cochlear nucleus (VCN) of adult E2a-Mover+/+ mice, and absent in E2a-Mover-/- animals. 74

Figure 2: Mover colocalizes with presynaptic markers at synapses contacting bushy cells. 75 Figure 3: Synaptic vesicles at Endbulbs of Held have more Mover per SV than inhibitory synapses contacting bushy cells.

Figure 4: Mover localizes more to the active zone in inhibitory synapses. 79 
Wallrafen, 2019 


\section{DECLARATION}

Herewith I declare, that I prepared the doctoral thesis "The presynaptic protein Mover is heterogeneously expressed across brain areas and synapse types" on my own and with no other sources and aids than quoted.

Göttingen, $20^{\text {th }}$ of June 2019.

Rebecca Wallrafen 
Wallrafen, 2019 